\title{
Reference ranges ("normal values") for cardiovascular magnetic resonance (CMR) in adults and children: 2020 update
}

\author{
Nadine Kawel-Boehm ${ }^{1,2}$, Scott J. Hetzel ${ }^{3}$, Bharath Ambale-Venkatesh ${ }^{4}$, Gabriella Captur ${ }^{5,6}$, \\ Christopher J. Francois ${ }^{7}$, Michael Jerosch-Herold ${ }^{8}$, Michael Salerno $^{9}$, Shawn D. Teague ${ }^{10}$, \\ Emanuela Valsangiacomo-Buechel ${ }^{11}$, Rob J. van der Geest ${ }^{12}$ and David A. Bluemke ${ }^{7^{*}}$ (D)
}

\begin{abstract}
Cardiovascular magnetic resonance (CMR) enables assessment and quantification of morphological and functional parameters of the heart, including chamber size and function, diameters of the aorta and pulmonary arteries, flow and myocardial relaxation times. Knowledge of reference ranges ("normal values") for quantitative CMR is crucial to interpretation of results and to distinguish normal from disease. Compared to the previous version of this review published in 2015, we present updated and expanded reference values for morphological and functional CMR parameters of the cardiovascular system based on the peer-reviewed literature and current CMR techniques. Further, databases and references for deep learning methods are included.
\end{abstract}

Keywords: Normal values, Reference range, Cardiac magnetic resonance

\section{Background}

Cardiovascular magnetic resonance (CMR) provides a wealth of information to help distinguish health from disease. In addition to non-invasively defining chamber sizes and global function, CMR can also assess regional cardiac function as well as tissue composition (myocardial $\mathrm{T} 1, \mathrm{~T} 2$ and $\mathrm{T} 2 *$ relaxation time). Advantages of quantitative evaluation of CMR images are objective differentiation between pathology and normal conditions, grading of disease severity, monitoring changes during therapy and evaluating prognosis [1].

Knowledge of the range of normal structure and function is required to interpret abnormal cardiac conditions. Thus, the aim of this review is to provide reference

\footnotetext{
*Correspondence: dbluemke@wisc.edu

${ }^{7}$ Department of Radiology, University of Wisconsin School of Medicine and Public Health, 600 Highland Avenue, Madison, WI 53792, USA Full list of author information is available at the end of the article
}

intervals ("normal values") for morphological and functional CMR parameters of the cardiovascular system based on a systematic review of the literature using current CMR techniques and sequences.

Since the initial publication of the "normal value review" in 2015 [1], new research related to CMR reference values have been published and are now integrated in this update. Previous topics were expanded with new sections including morphological and functional parameters in athletes, myocardial T2 mapping, myocardial perfusion, left-ventricular (LV) trabeculation and normal dimensions of the pulmonary arteries in adults and children. Further, feature tracking is increasingly used to assess myocardial strain and reference intervals are now available for that technology. Deep learning methods are rapidly being incorporated into clinical software analysis packages $[2,3]$. These new analytic methods are expected to accelerate quantification of myocardial function from CMR images. To date, reference ranges based on cohorts of healthy subjects using deep learning methods have not give appropriate credit to the original author(s) and the source, provide a link to the Creative Commons licence, and indicate if changes were made. The images or other third party material in this article are included in the article's Creative Commons licence, unless indicated otherwise in a credit line to the material. If material is not included in the article's Creative Commons licence and your intended use is not permitted by statutory regulation or exceeds the permitted use, you will need to obtain permission directly from the copyright holder. To view a copy of this licence, visit http://creativecommons.org/licenses/by/4.0/. The Creative Commons Public Domain Dedication waiver (http://creativecommons.org/publicdomain/zero/1.0/) applies to the data made available in this article, unless otherwise stated in a credit line to the data. 
been presented. However due to the potential importance of this topic, we present algorithms and major references related to CMR on these methods.

\section{Methods}

A literature search was performed in PubMed to identify publications of CMR reference intervals for each section. When feasible (discussed further below), we sought to provide weighted means calculated based on these published normal values in healthy individuals. General criteria used for inclusion of data in this review are as follows:

a) Sample size of at least 40 subjects. 40 subjects is accepted as the smallest sample size that allows calculation of reference ranges using a parametric method for data with a Gaussian distribution [4]. In some circumstances, separate reference ranges need to be provided by gender. In that case, the sample size of included studies were at least a minimum of 40 subjects per gender. Exceptions to sample size of 40 subjects per group were made for clinically relevant parameters where no publication was available with sufficient sample size for certain parameters. However, reference ranges based on a smaller sample size are of limited validity and should be applied with caution.

b) Only values of "healthy" reference cohorts were included. In particular, reference cohorts that included subjects with a disease or condition known to affect the measured parameter (e.g. hypertension and diabetes) were excluded. For publications that described population statistics (e.g., the MESA study, UK Biobank), we used data only from subgroups of individuals without risk factors or conditions known to affect the CMR parameter. In cases where the original manuscript did not provide sufficient information to allow upper and lower limits to be calculated, authors were contacted for clarification.

c) If two or more publications were determined to refer to the same healthy reference cohort, the values of the cohort were included only once.

Manuscripts were then excluded from consideration as follows: (a) obsolescent CMR technique, (b) missing data that were not provided by the authors of the original publication on request and/ or (c) insufficient or inconsistent description of methods and/or (d) methods of analysis that were not consistent with current Society for Cardiovascular Magnetic Resonance (SCMR) guidelines [5] as of the time of this review.

Technical factors such as sequence parameters are relevant for CMR, and these factors are provided in relationship to the reference values. In addition, factors related to post processing will affect the CMR analysis and these factors are also described. Finally, when available, the relationship of demographic factors (e.g. age, gender, and ethnicity) to reference values are described in each section.

\section{Statistical methods}

Statistical analyses were performed with $\mathrm{R}$ for statistical computing (version 3.5, R Core Team, Vienna, Austria). Results from multiple studies reporting normal values for the same CMR parameters were combined using a random effects meta-analysis model as implemented by the metamean function in the meta library in R. This produced a weighted, pooled estimate of the population mean of the CMR parameters in the combined studies. Upper and lower limits of normal values were calculated as $\pm 2 \mathrm{SDp}$, where SDp is the pooled standard deviation calculated from the standard deviations reported in each study. Mean values and limits of normal values were "rounded up" to avoid excess digits beyond the measurement capability of CMR.

\section{Left ventricular dimensions and functions in the adult \\ CMR acquisition parameters}

The primary method used to assess the LV is balanced steady-state free precession (bSSFP) technique at 1.5 or $3 \mathrm{~T}$ CMR (Table 1). bSSFP technique yields improved blood-myocardial contrast compared to its predecessor, fast gradient echo (FGRE) sequence.

\section{CMR analysis methods}

Papillary muscle mass has been shown to significantly affect LV volumes and mass [6-8]. No uniformly accepted convention has been used for analyzing trabeculation and papillary muscle mass. Post-processing recommendations by the SCMR [9] stipulate that papillary muscles should either be consistently included in the LV volume or in the LV mass, but not in both. Tables of normal values should specify the status of the papillary muscles in the CMR analysis.

The majority of published articles used semi-automatic software for analysis of LV function and structure [10-16]. Short-axis images are most commonly analyzed on a perslice basis, deriving LV mass and volume by applying the Simpson's method ("stack of disks") [17]. An example of LV contouring is shown in Fig. 1. Automated CMR analysis facilitated by machine learning is rapidly making inroads in LV volume and mass quantification [3]. The primary focus of early manuscripts has been on agreement between manual and automatic contouring [2]. However, to date, CMR variables for healthy cohorts have not been reported using machine learning methods. 
Table 1 References, normal adult left ventricular volumes, function and dimensions

\begin{tabular}{llll}
\hline First author, year & CMR technique & n, male:female & Age range (years) \\
\hline Hudsmith, 2005 [22] & 1.5 T, short axis bSSFP, papillary muscles included in LV mass & $63: 45$ & $21-68$ \\
Maceira, 2006 [10] & 1.5 T, short axis bSSFP, papillary muscles included in LV mass & $60: 60$ & $20-80$ \\
Chang, 2012 [23] & 1.5 T, short axis bSSFP, papillary muscles included in LV volume & $64: 60$ & $20-70$ \\
Macedo, 2013 [24] & 1.5 T, short axis bSSFP, papillary muscles included in LV mass & $54: 53$ & $20-80$ \\
Yeon, 2015 [25] & 1.5 T, short axis bSSFP, papillary muscles included in LV volume & $512: 340$ & $(61 \pm 9)^{\mathrm{a}}$ \\
Le, 2016 [11] & 3T, short axis bSSFP, papillary muscles included in LV mass & $91: 89$ & $20-69$ \\
Le Ven, 2016 [14] & 1.5 T, Short axis bSSFP, papillary muscles included in LV mass & $196: 238$ & $18-36$ \\
Lei, 2017 [15] & 3T, short axis bSSFP, papillary muscles included in LV volume & $60: 60$ & $23-83$ \\
Petersen, 2017 [16] & 1.5 T, short axis bSSFP, papillary muscles included in LV volume & $368: 432$ & $45-74$ \\
Bentatou, 2018 [12] & 1.5 T, short axis bSSFP, papillary muscles included in LV mass & $70: 70$ & $20-69$ \\
Buelow, 2018 [13] & 1.5 T, short axis bSSFP, papillary muscles included in LV mass & $291: 326$ & $20-80^{b}$ \\
Liu, 2018 [26] & 1.5 T, short axis bSSFP, papillary muscles included in LV mass & $50: 50$ & $20-70$ \\
\hline
\end{tabular}

$n$ number of study subjects, $b S S F P$ balanced steady-state free precession, $L V$ left ventricle

${ }^{a}$ Mean \pm SD (age-range not provided in original publication)

${ }^{\mathrm{b}} 6$ subjects $>80$ years included

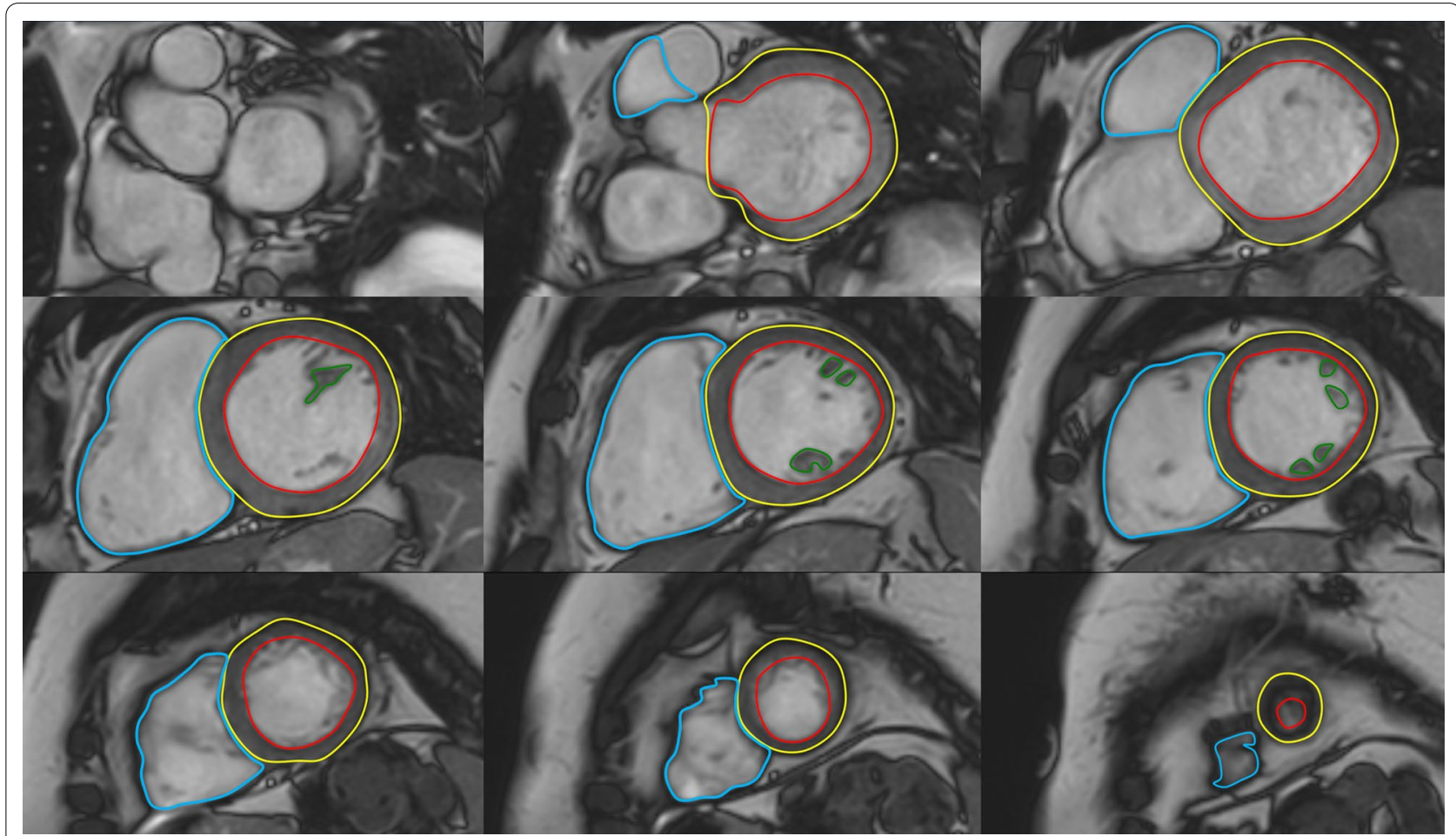

Fig. 1 Contouring of the left ventricle (LV) and right ventricle (RV). Note that LV papillary muscle mass has been isolated and added to LV mass. RV papillary muscles and trabeculations were included in the RV volume

Measurements of LV diameter obtained on cine bSSFP images at diastole and systole on a 4 chamber view and short axis view are shown in Fig. 2.

\section{Demographic parameters}

Gender is independently related to ventricular volumes and mass. Absolute and normalized volumes decrease 


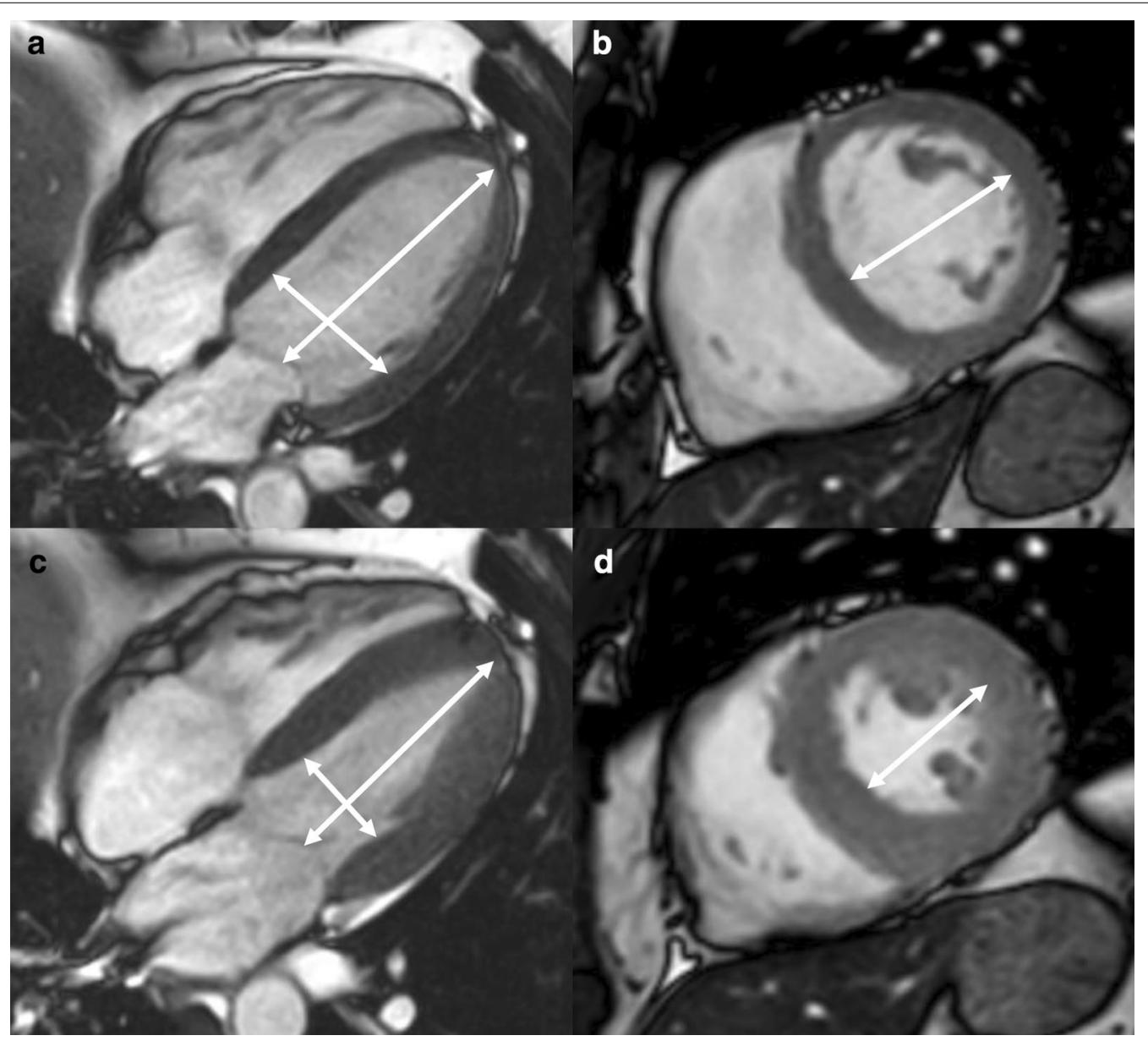

Fig. 2 Measurements of LV diameters obtained on cine bSSFP images during diastole $(\mathbf{a}, \mathbf{b})$ and systole (c, d) on the 4 chamber view (a, c) and short axis view $(\mathbf{b}, \mathbf{d})$. The longitudinal diameter of the LV was measured on the 4 chamber view as the distance between the mitral valve plane and the LV apex $(\mathbf{a}, \mathbf{c})$. On the 4 chamber view the transverse diameter was defined as the distance between the septum and the lateral wall at the basal level [18]. On the short axis view the transverse diameter was obtained at the level of the basal papillary muscles (b, d) [15]

in relationship to age in adults [10] in a continuous manner. For convenience, both average, and values per age decile are given in Tables 2, 3, 4 and 5 based on the peerreviewed literature.

\section{Studies included in this review}

Multiple studies have presented cohorts of normal individuals for determining normal LV dimensions. For the purpose of this review, only cohorts of 40 or more normal subjects stratified by gender using bSSFP CMR technique at 1.5 or $3 \mathrm{~T}$ have been included. In addition, a full description of the subject cohort (including the analysis methods used), age and gender of subjects was required to be included for this review. Two studies $[18,19]$ included papillary muscles in LV volume except if directly attached to the LV wall, in which case they were included in LV mass (LVM) instead. Since this approach was inconsistent with post-processing recommended by SCMR [9] and other manuscripts on the topic, both studies were excluded from the current analysis. Data at 1.5 and $3 \mathrm{~T}$ is now available for normal subjects using bSSFP short axis imaging. Since it has been shown that parameters of LV volumes and function do not vary by field strength, calculation of the weighted means of these parameters include studies performed at $1.5 \mathrm{~T}$ and $3 \mathrm{~T}$ [20]. Information on ethnicity in relationship to LV parameters is not available for the majority of papers reporting the bSSFP technique and is therefore not reported in this review. However, small differences in LV parameters by ethnicity have been reported in the Multi-ethnic Study of Atherosclerosis (MESA) study; for further information on the magnitude of such 
Table 2 Left ventricular parameters in the adult for men and women (ages 18-83), papillary muscles included in left ventricular mass

\begin{tabular}{|c|c|c|c|c|c|c|c|c|}
\hline \multirow[t]{2}{*}{ Parameter } & \multicolumn{4}{|l|}{ Men } & \multicolumn{4}{|c|}{ Women } \\
\hline & $n$ & Mean $_{p}$ & $S D_{p}$ & $\mathrm{LL}^{-U L^{h}}$ & $n$ & Mean $_{p}$ & $S D_{p}$ & $\mathrm{LL}^{-U \mathrm{~L}^{\mathrm{h}}}$ \\
\hline $\operatorname{LVEDV~}(\mathrm{ml})^{\mathrm{a}}$ & 464 & 155 & 30 & $95-215$ & 485 & 123 & 22 & $78-167$ \\
\hline LVEDV/BSA $\left(\mathrm{ml} / \mathrm{m}^{2}\right)^{b}$ & 875 & 79 & 15 & $50-108$ & 931 & 73 & 12 & $50-96$ \\
\hline LVESV $(m l)^{a}$ & 464 & 55 & 15 & $25-85$ & 485 & 43 & 11 & $21-64$ \\
\hline LVESV/BSA $\left(\mathrm{ml} / \mathrm{m}^{2}\right)^{\mathrm{b}}$ & 875 & 29 & 9 & $11-47$ & 931 & 25 & 7 & $10-40$ \\
\hline $\operatorname{LVSV}(\mathrm{ml})^{c}$ & 410 & 103 & 21 & $61-145$ & 432 & 83 & 16 & $52-114$ \\
\hline LVSV/BSA $\left(\mathrm{ml} / \mathrm{m}^{2}\right)^{\mathrm{d}}$ & 701 & 52 & 10 & $33-72$ & 758 & 49 & 8 & $33-64$ \\
\hline $\operatorname{LVEF}(\%)^{b}$ & 875 & 64 & 8 & 49-79 & 931 & 66 & 7 & $52-79$ \\
\hline $\operatorname{LVM}(g)^{a}$ & 464 & 121 & 28 & $66-176$ & 485 & 83 & 21 & $41-125$ \\
\hline LVM/BSA $\left(\mathrm{g} / \mathrm{m}^{2}\right)^{\mathrm{e}}$ & 805 & 62 & 11 & $39-85$ & 861 & 49 & 10 & $30-68$ \\
\hline $\operatorname{LVCO}(I / \mathrm{min})^{f}$ & 91 & 5.6 & 1.1 & $3.4-7.8$ & 89 & 4.5 & 0.9 & $2.7-6.3$ \\
\hline $\operatorname{LVCl}\left(1 / \mathrm{min} / \mathrm{m}^{2}\right)^{\mathrm{f}}$ & 91 & 3.0 & 0.6 & $1.8-4.2$ & 89 & 2.9 & 0.5 & $1.9-3.9$ \\
\hline LVM/LVEDV $(\mathrm{g} / \mathrm{ml})^{\mathrm{g}}$ & 287 & 0.7 & 0.1 & $0.4-0.9$ & 327 & 0.6 & 0.1 & $0.3-0.8$ \\
\hline
\end{tabular}

$n$ number of study subjects included in the weighted mean values, mean ${ }_{p}$ pooled weighted mean, $S D_{p}$ pooled standard deviation, $L L$ lower limit, $U L$ upper limit, $L V$ left ventricular, EDV end-diastolic volume, ESV end-systolic volume, SV stroke volume, EF ejection fraction, $L V M$ left ventricular mass, CO cardiac output, Cl cardiac index, $B S A$ body surface area

a Pooled weighted values from references $[10,11,14,22,24]$

${ }^{b}$ Pooled weighted values from references [10-14, 22, 24, 26]

c Pooled weighted values from references [10, 11, 14, 22]

${ }^{d}$ Pooled weighted values from references $[10,11,13,14,22]$

e Pooled weighted values from references $[10,11,13,14,22,24,26]$

f Values from reference [11]

${ }^{g}$ Pooled weighted values from references $[11,14]$

${ }^{\mathrm{h}}$ Calculated as mean $\mathrm{p}_{\mathrm{p}} \pm 2 * \mathrm{SD}_{\mathrm{p}}$

differences, the reader is referred to the work by Natori $\mathrm{S}$ et al. [21].

Normal adult values for LV dimensions and functions according to those studies that consistently included papillary muscles in the LVM are presented in Tables 2, 3, 4, whereas those that consistently included papillary muscles in the LV volume are presented in Table 5. For parameters with sufficient sample size, values are also presented per age decile (Tables 3, 4).

\section{Additional left ventricular function parameters}

In addition to left ventricular ejection fraction (LVEF), Maceira et al. have provided additional functional parameters that may be useful in some settings [10]. These are summarized in Table 6. For diastolic function, the derivative of the time/volume filling curve expresses the peak filling rate (PFR). Both early (E) and active (A) transmitral filling rates are provided. In addition, longitudinal atrioventricular plane descent (AVPD) and sphericity index (volume observed/volume of sphere using long axis as diameter) at end diastole and end systole are given. These latter parameters are not routinely used for clinical diagnosis. A number of publications have also reported LV end-diastolic and end-systolic diameters by CMR; these parameters are summarized in Table 7.

\section{Right ventricular dimensions and functions in the adult \\ CMR acquisition parameters}

For measurement of right ventricular (RV) volumes, a stack of cine bSSFP images is acquired either in the short axis plane or transaxial plane [9].

\section{CMR analysis methods}

Similar to the LV, analysis of the RV is usually performed on a per slice basis by manual contouring of the endocardial and epicardial borders. Volumes are calculated based on the Simpson's method [17]. The RV volumes and mass are significantly affected by inclusion or exclusion of trabeculations and papillary muscles [27, 28]. For manual contouring, inclusion of trabeculations and papillary muscles as part of the RV volume will achieve higher reproducibility $[9,27,28]$. However, semiautomatic software is increasingly used for volumetric analysis, enabling automatic delineation of papillary muscles [29]. Therefore, normal values for both methods are provided. An example for RV contouring is shown in Fig. 1. 


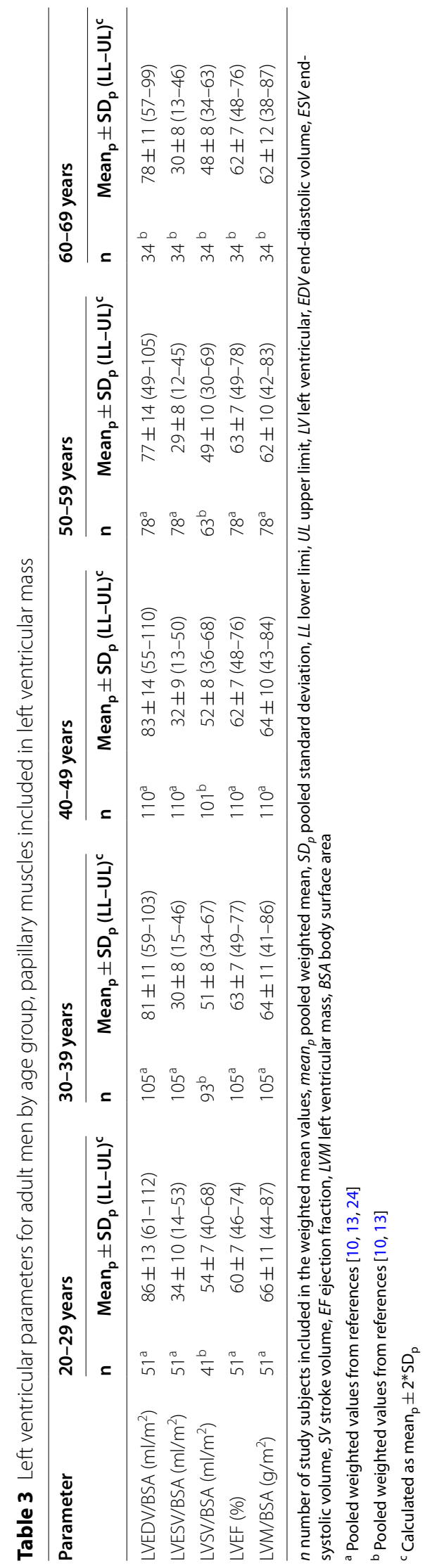




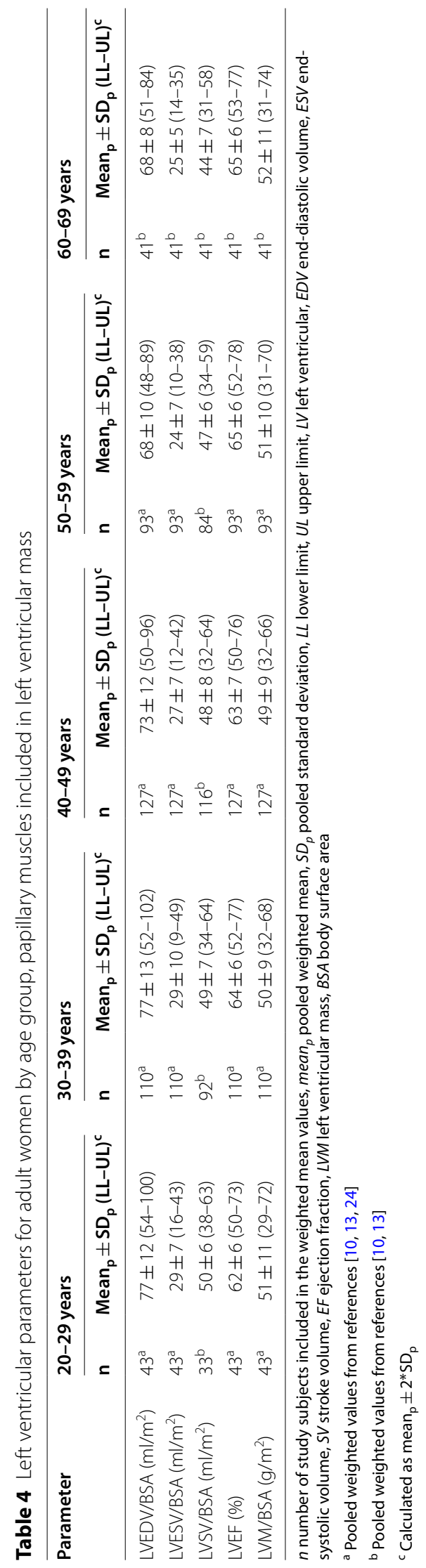


Table 5 Left ventricular parameters in the adult for men and women (ages 16-83), papillary muscles included in left ventricular volume

\begin{tabular}{|c|c|c|c|c|c|c|c|c|}
\hline \multirow[t]{2}{*}{ Parameter } & \multicolumn{4}{|c|}{ Men } & \multicolumn{4}{|c|}{ Women } \\
\hline & $n$ & $\operatorname{Mean}_{\mathrm{p}}$ & $S D_{p}$ & LL-ULg & $\mathrm{n}$ & $\operatorname{Mean}_{\mathrm{p}}$ & $S D_{p}$ & LL-UL ${ }^{g}$ \\
\hline $\operatorname{LVEDV~}(\mathrm{ml})^{\mathrm{a}}$ & 832 & 145 & 31 & $83-207$ & 1064 & 112 & 21 & $70-155$ \\
\hline LVEDV/BSA $\left(\mathrm{ml} / \mathrm{m}^{2}\right)^{\mathrm{b}}$ & 832 & 77 & 15 & $47-107$ & 1064 & 69 & 12 & $45-93$ \\
\hline $\operatorname{LVESV}(\mathrm{ml})^{\mathrm{a}}$ & 832 & 53 & 18 & $19-88$ & 1064 & 39 & 12 & $15-64$ \\
\hline LVESV/BSA $\left(\mathrm{ml} / \mathrm{m}^{2}\right)^{\mathrm{b}}$ & 832 & 29 & 9 & $11-47$ & 1064 & 24 & 7 & $10-38$ \\
\hline $\operatorname{LVSV}(\mathrm{ml})^{\mathrm{a}}$ & 832 & 91 & 18 & $55-127$ & 1064 & 73 & 13 & $47-99$ \\
\hline LVSV/BSA $\left(\mathrm{ml} / \mathrm{m}^{2}\right)^{\mathrm{c}}$ & 772 & 48 & 9 & $30-66$ & 1004 & 45 & 7 & $30-59$ \\
\hline $\operatorname{LVEF}(\%)^{b}$ & 832 & 63 & 6 & $51-76$ & 1064 & 66 & 7 & $52-79$ \\
\hline $\operatorname{LVM}(g)^{a}$ & 832 & 105 & 24 & $57-152$ & 1064 & 73 & 15 & 43-103 \\
\hline LVM/BSA $\left(\mathrm{g} / \mathrm{m}^{2}\right)^{\mathrm{b}}$ & 832 & 56 & 10 & $36-75$ & 1064 & 45 & 7 & $30-59$ \\
\hline $\operatorname{LVCO}(1 / \mathrm{min})^{d}$ & 464 & 6.1 & 1.1 & $3.9-8.3$ & 632 & 4.9 & 1.0 & $3.0-6.9$ \\
\hline $\operatorname{LVCI}\left(\mathrm{I} / \mathrm{min} / \mathrm{m}^{2}\right)^{\mathrm{e}}$ & 404 & 3.2 & 0.6 & $2.1-4.3$ & 572 & 2.9 & 0.5 & $1.9-4.0$ \\
\hline LVM/LVEDV $(\mathrm{g} / \mathrm{ml})^{\mathrm{f}}$ & 708 & 0.7 & 0.2 & $0.3-1.2$ & 944 & 0.7 & 0.1 & $0.4-1.0$ \\
\hline
\end{tabular}

$n$ number of study subjects included in the weighted mean values, mean $p$ pooled weighted mean, $S D_{p}$ pooled standard deviation, $L V$ left ventricular, $E D V$ end-diastolic volume, ESV end-systolic volume, SV stroke volume, EF ejection fraction, LVM left ventricular mass, CO cardiac output, CI cardiac index, BSA body surface area

a Pooled weighted values from references $[15,16,19,23,25]$

${ }^{b}$ Pooled weighted values from references $[15,16,18,19,23,25]$

' Pooled weighted values from references $[16,18,19,23,25]$

$d$ Pooled weighted values from references $[15,23,25]$

e Pooled weighted values from references $[23,25]$

${ }^{f}$ Pooled weighted values from references $[16,25]$

${ }^{g}$ Calculated as mean ${ }_{p} \pm 2 * \mathrm{SD}_{\mathrm{p}}$

Detailed recommendations for RV acquisitions and post processing have been published [9].

\section{Demographic parameters}

RV mass and volumes are dependent on body surface area (BSA) [14, 29]. Absolute and RV volumes indexed by BSA are significantly larger in males compared to females $[11,14,16,18,22,29]$. Further, RV volumes decrease with greater age $[11,14,16,18,22,29]$.

\section{Studies included in this review}

Criteria regarding study inclusion are identical compared to the LV. Nine studies based on bSSFP imaging were included (Table 8). In one study, papillary muscles were included as part of the RV mass and excluded from the RV volume [29] with results presented for men and women in Table 9. In the remaining eight studies, the papillary muscles were included as part of the RV cavity volume rather than included in the RV mass [11, 14-16, $18,22-24]$ with pooled weighted mean values presented for men and women (Table 10). For a subset of three of these studies [18, 23, 24], for parameters with a sufficient sample size pooled weighted mean values are presented based on age deciles between 20 and 59 years of age for both men (Table 11) and women (Table 12).

\section{Additional RV function parameters}

Similar to the LV, Maceira et al. have provided additional functional parameters, including early and active peak filling rate and the longitudinal AVPD, that may have relevance to specific applications and can be found in the original publication [29].

\section{Left atrial dimensions and functions in the adult CMR acquisition parameters}

There is limited consensus in the literature about how to measure left atrial (LA) volume. The most common methods to measure LA volume are the modified Simpson's method (analogous to that used to measure LV and RV volumes) and the biplane area-length method [30]. Dedicated 3-dimensional modeling software has also been employed [31].

In the Simpson's method, a stack of cine bSSFP images either in the SAx, the horizontal long axis or transverse view, is required. For 3-dimensional modeling a stack of SAx images has been used [31]. Evaluation by the biplane area-length method is based on a 2 and 4 chamber view $[11,16,32-34]$.

LA longitudinal and transverse diameters and area have been measured on 2, 3, and 4 chamber cine bSSFP images [31, 33, 35] (Fig. 3). 
Table 6 Functional and geometric parameters of the normal left ventricle in the adult, from reference [10]

\begin{tabular}{|c|c|c|c|c|c|c|}
\hline \multirow[t]{2}{*}{ Parameter } & \multicolumn{3}{|c|}{ Men $(n=60)$} & \multicolumn{3}{|c|}{ Women $(n=60)$} \\
\hline & Mean & SD & LL-ULa & Mean & SD & LL-UL ${ }^{a}$ \\
\hline $\operatorname{PFRE}(\mathrm{ml} / \mathrm{s})$ & 527 & 140 & $247-807$ & 477 & 146 & $185-769$ \\
\hline PFRE /BSA $\left(\mathrm{ml} / \mathrm{m}^{2}\right)$ & 270 & 70 & $130-410$ & 279 & 81 & $117-441$ \\
\hline PFRE/EDV (/s) & 3.4 & 0.7 & $2.0-4.8$ & 3.8 & 0.8 & $2.2-5.4$ \\
\hline PFRA (ml/s) & 373 & 82 & 209-537 & 283 & 69 & $145-421$ \\
\hline PFRA/BSA $\left(\mathrm{ml} / \mathrm{m}^{2}\right)$ & 193 & 44 & $105-281$ & 168 & 44 & $80-256$ \\
\hline PFRA/EDV (/s) & 2.6 & 0.6 & $1.4-3.8$ & 2.3 & 0.5 & $1.3-3.3$ \\
\hline PFRE/PFRA & 1.4 & 0.3 & $0.8-2.0$ & 1.7 & 0.3 & $1.1-2.3$ \\
\hline Septal AVPD (mm) & 15 & 4 & $7-23$ & 14 & 3 & $8-20$ \\
\hline Septal AVPD /long length (\%) & 15 & 3 & $9-21$ & 16 & 4 & $8-24$ \\
\hline Lateral AVPD (mm) & 18 & 4 & $10-26$ & 17 & 3 & $11-23$ \\
\hline Lateral AVPD /long length (\%) & 17 & 3 & $11-23$ & 19 & 3 & $13-25$ \\
\hline Sphericity index, diastole ${ }^{b}$ & 0.31 & 0.07 & $0.20-0.48$ & 0.34 & 0.07 & $0.20-0.48$ \\
\hline Sphericity index, systole & 0.20 & 0.05 & $0.1-0.3$ & 0.23 & 0.07 & $0.09-0.37$ \\
\hline
\end{tabular}

$n$ number of study subjects, SD standard deviation, $L L$ lower limit, UL upper limit, BSA body surface area, PFR peak filling rate, E early, $A$ active, $A V P D$ atrioventricular plane descent

${ }^{\text {a }}$ Calculated as mean $\pm 2 * S D$

${ }^{b}$ Pooled weighted mean and SD calculated from references $[10,18]$ with $n=195$ men and $n=233$ women.

Table 7 Left ventricular diameters in the adult for men and women, bSSFP technique

\begin{tabular}{|c|c|c|c|c|c|c|c|c|}
\hline \multirow[t]{2}{*}{ Parameter } & \multicolumn{4}{|c|}{ Men } & \multicolumn{4}{|c|}{ Women } \\
\hline & $\mathrm{n}$ & Mean $_{p}$ & $S D_{p}$ & $\mathrm{LL}^{-U L^{\mathrm{e}}}$ & $\mathrm{n}$ & Mean $_{p}$ & $S D_{p}$ & LL-UL \\
\hline LV end-diastolic diameter $4 \mathrm{Ch}(\mathrm{mm})^{\mathrm{a}}$ & 227 & 52 & 5 & $42-62$ & 188 & 49 & 5 & $39-59$ \\
\hline LV end-diastolic diameter SAx $(\mathrm{mm})^{\mathrm{b}}$ & 400 & 53 & 5 & $44-62$ & 572 & 49 & 4 & $41-57$ \\
\hline LV end-systolic diameter $4 \mathrm{Ch}(\mathrm{mm})^{c}$ & 54 & 32 & 3 & $26-38$ & 53 & 28 & 6 & $16-40$ \\
\hline LV end-systolic diameter SAx (mm) ${ }^{d}$ & 60 & 34 & 3 & $28-40$ & 60 & 31 & 4 & $23-39$ \\
\hline
\end{tabular}

bSSFP balanced steady-state free precession, $n$ number of study subjects included in the weighted mean values, mean ${ }_{p}$ pooled weighted mean, $S D_{p}$ pooled standard deviation, $L L$ lower limit, $U L$ upper limit, $L V$ left ventricular, $4 C h 4$ chamber view, SAx short axis

a Pooled weighted values from references $[18,24]$

${ }^{b}$ Pooled weighted values from references $[15,25]$

'Values from reference [24]

d Values from reference [15]

${ }^{\mathrm{e}}$ Calculated as mean $_{\mathrm{p}} \pm 2 * \mathrm{SD}_{\mathrm{p}}$

Table 8 References, normal right ventricular volumes, function and dimensions in the adult

\begin{tabular}{llll}
\hline First author, year & CMR technique & n, male:female & Age range (years) \\
\hline Hudsmith, 2005 [22] & 1.5 T, short axis bSSFP, papillary muscles included in RV volume & $63: 45$ & $21-68$ \\
Maceira, 2006 [29] & 1.5 T, short axis bSSFP, papillary muscles included in RV mass & $60: 60$ & $20-80$ \\
Chang, 2012 [23] & 1.5 T, short axis bSSFP, papillary muscles included in RV volume & $64: 60$ & $20-70$ \\
Macedo, 2013 [24] & 1.5 T, short axis bSSFP, papillary muscles included in RV volume & $54: 53$ & $20-80$ \\
Le Ven, 2015 [14] & 1.5 T, short axis bSSFP, papillary muscles included in RV volume & $196: 238$ & $18-36$ \\
Lei, 2016 [15] & 3T, short axis bSSFP, papillary muscles included in RV volume & $60: 60$ & $23-83$ \\
Le, 2016 [11] & 3T, short axis bSSFP, papillary muscles included in RV volume & $91: 89$ & $20-69$ \\
Aquaro, 2017 [18] & 1.5 T, short axis bSSFP, papillary muscles included in RV volume & $173: 135$ & $16->60$ \\
Petersen, 2017 [16] & 1.5 T, short axis bSSFP, papillary muscles included in RV volume & $368: 432$ & $45-74$
\end{tabular}

$n$ number of study subjects, $b S S F P$ balancedsteady-state free precession, $R V$ right ventricular 
Table 9 Right ventricular parameters in the adult for men and women (ages 20-79), papillary muscles included in right ventricular mass, from reference [29]

\begin{tabular}{|c|c|c|c|c|c|c|}
\hline \multirow[t]{2}{*}{ Parameter } & \multicolumn{3}{|c|}{ Men $(n=60)$} & \multicolumn{3}{|c|}{ Women $(n=60)$} \\
\hline & Mean & SD & LL-UL ${ }^{a}$ & Mean & SD & LL-ULa \\
\hline RVEDV (ml) & 163 & 27 & $109-217$ & 127 & 24 & $79-175$ \\
\hline RVEDV/BSA $\left(\mathrm{ml} / \mathrm{m}^{2}\right)$ & 83 & 13 & 58-109 & 74 & 12 & $51-97$ \\
\hline RVESV (ml) & 57 & 17 & $23-91$ & 44 & 15 & $13-75$ \\
\hline RVESV/BSA $\left(\mathrm{ml} / \mathrm{m}^{2}\right)$ & 29 & 9 & $12-46$ & 26 & 8 & $9-42$ \\
\hline RVSV (ml) & 106 & 18 & $71-141$ & 83 & 13 & $56-110$ \\
\hline RVSV/BSA $\left(\mathrm{ml} / \mathrm{m}^{2}\right)$ & 54 & 8 & $38-71$ & 48 & 7 & $35-61$ \\
\hline RVEF (\%) & 66 & 7 & $51-80$ & 66 & 7 & $52-80$ \\
\hline RVM (g) & 66 & 15 & $37-95$ & 48 & 11 & $26-71$ \\
\hline RVM/BSA ( $\left.\mathrm{g} / \mathrm{m}^{2}\right)$ & 34 & 7 & $20-48$ & 28 & 6 & $16-40$ \\
\hline
\end{tabular}

$n$ number of study subjects, $S D$ standard deviation, $L L$ lower limit, $U L$ upper limit, $R V$ right ventricular, EDV end-diastolic volume, ESV end-systolic volume, $S V$ stroke volume, EF ejection fraction, $R V M$ right ventricular mass, BSA body surface area

${ }^{\text {a }}$ Calculated as mean $\pm 2 * S D$

Table 10 Right ventricular parameters in the adult for men and women (ages 20-83), papillary muscles included in right ventricular volume

\begin{tabular}{|c|c|c|c|c|c|c|c|c|}
\hline \multirow[t]{2}{*}{ Parameter } & \multicolumn{4}{|l|}{ Men } & \multicolumn{4}{|c|}{ Women } \\
\hline & $\mathbf{n}$ & Meanp & SDp & LL-ULg & $n$ & Meanp & SDp & LL-ULg \\
\hline $\operatorname{RVEDV}(\mathrm{ml})^{\mathrm{a}}$ & 896 & 166 & 39 & $87-244$ & 977 & 122 & 27 & $68-176$ \\
\hline RVEDV/BSA $\left(\mathrm{ml} / \mathrm{m}^{2}\right)^{\mathrm{b}}$ & 1069 & 88 & 17 & $53-123$ & 1112 & 76 & 14 & $48-104$ \\
\hline $\operatorname{RVESV}(m l)^{a}$ & 896 & 73 & 22 & $29-117$ & 977 & 50 & 15 & $20-80$ \\
\hline RVESV/BSA $\left(\mathrm{ml} / \mathrm{m}^{2}\right)^{\mathrm{b}}$ & 1069 & 38 & 11 & $17-59$ & 1112 & 30 & 9 & $13-48$ \\
\hline $\operatorname{RVSV}(\mathrm{ml})^{c}$ & 842 & 95 & 26 & $43-146$ & 924 & 74 & 18 & 39-109 \\
\hline RVSV/BSA $\left(\mathrm{ml} / \mathrm{m}^{2}\right)^{\mathrm{d}}$ & 955 & 52 & 12 & $28-75$ & 999 & 48 & 9 & $29-66$ \\
\hline $\operatorname{RVEF}(\%)^{b}$ & 1069 & 57 & 8 & $42-72$ & 1112 & 60 & 7 & $46-74$ \\
\hline $\operatorname{RVM}(g)^{e}$ & 117 & 36 & 9 & $17-54$ & 98 & 30 & 9 & $13-48$ \\
\hline RVM/BSA $\left(\mathrm{g} / \mathrm{m}^{2}\right)^{\mathrm{e}}$ & 117 & 19 & 4 & $10-28$ & 98 & 17 & 5 & $7-28$ \\
\hline $\operatorname{RVCO}(1 / \min )^{f}$ & 155 & 5.6 & 1.4 & $2.8-8.3$ & 149 & 4.4 & 1.0 & $2.4-6.4$ \\
\hline $\operatorname{RVCl}\left(1 / \mathrm{min} / \mathrm{m}^{2}\right)^{f}$ & 155 & 3.0 & 0.7 & $1.5-4.5$ & 149 & 2.8 & 0.6 & $1.6-4.0$ \\
\hline
\end{tabular}

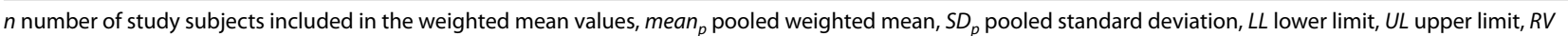
right ventricular, EDV end-diastolic volume, $E S V$ end-systolic volume, SV stroke volume, $E F$ ejection fraction, $R V M$ right ventricular mass, $C O$ cardiac output, $C I$ cardiac index, BSA body surface area

a Pooled weighted values from references [11, 14-16, 22-24]

${ }^{b}$ Pooled weighted values from references [11, 14-16, 18, 22-24]

' Pooled weighted values from references [11, 14-16, 22, 23]

d Pooled weighted values from references [11, 14, 16, 18, 22, 23]

e Pooled weighted values from references $[22,24]$

${ }^{f}$ Pooled weighted values from references $[11,23]$

${ }^{g}$ Calculated as mean ${ }_{p} \pm 2 * \mathrm{SD}_{\mathrm{p}}$

\section{CMR analysis methods}

In many studies the LA appendage has been included as part of the LA volume and pulmonary veins are excluded $[14,31]$, but the practice of excluding both structures from the LA volume is increasingly gaining acceptance $[11,16,32,34]$.
The maximal LA volume is achieved during ventricular systole. In a cine acquisition, the maximum volume image can be defined as last image immediately before opening of the mitral valve. Accordingly the minimal LA volume image can be defined as the first image after closure of the mitral valve [36]. 
Table 11 Right ventricular parameters for adult men by age group, papillary muscles included in right ventricular volume

\begin{tabular}{|c|c|c|c|c|c|c|c|c|}
\hline \multirow[t]{2}{*}{ Parameter } & \multicolumn{2}{|c|}{ 20-29 years } & \multicolumn{2}{|c|}{$30-39$ years } & \multicolumn{2}{|c|}{$40-49$ years } & \multicolumn{2}{|c|}{ 50-59 years } \\
\hline & $\mathrm{n}$ & $\operatorname{Mean}_{\mathrm{p}} \pm \mathrm{SD}_{\mathrm{p}}(\mathrm{LL}-\mathrm{UL})^{\mathrm{c}}$ & n & $\operatorname{Mean}_{p} \pm S D_{p}(L L-U L)^{c}$ & $\mathrm{n}$ & $\operatorname{Mean}_{p} \pm \mathrm{SD}_{\mathrm{p}}(\mathrm{LL}-\mathrm{UL})^{\mathrm{c}}$ & $n$ & $\operatorname{Mean}_{\mathrm{p}} \pm \mathrm{SD}_{\mathrm{p}}(\mathrm{LL}-\mathrm{UL})^{*}$ \\
\hline RVEDV/BSA $\left(\mathrm{ml} / \mathrm{m}^{2}\right)^{\mathrm{a}}$ & 50 & $94 \pm 15(63-124)$ & 55 & $83 \pm 13(57-109)$ & 49 & $81 \pm 16(50-112)$ & 55 & $80 \pm 16(48-111)$ \\
\hline RVESV/BSA $\left(\mathrm{ml} / \mathrm{m}^{2}\right)^{\mathrm{a}}$ & 50 & $44 \pm 11(23-66)$ & 55 & $38 \pm 8(22-53)$ & 49 & $34 \pm 8(18-49)$ & 55 & $35 \pm 10(16-54)$ \\
\hline $\mathrm{RVSV} / \mathrm{BSA}\left(\mathrm{ml} / \mathrm{m}^{2}\right)^{\mathrm{b}}$ & 40 & $51 \pm 13(26-77)$ & 43 & $46 \pm 10(27-65)$ & 40 & $44 \pm 11(23-65)$ & 40 & $51 \pm 13(24-78)$ \\
\hline $\operatorname{RVEF}(\%)^{\mathrm{a}}$ & 50 & $52 \pm 8(36-69)$ & 55 & $55 \pm 7(41-68)$ & 49 & $57 \pm 8(40-73)$ & 55 & $57 \pm 8(41-74)$ \\
\hline
\end{tabular}

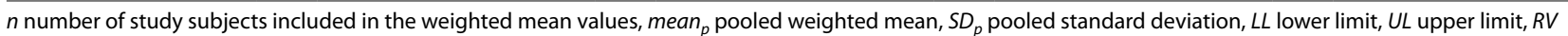
right ventricular, EDV end-diastolic volume, ESV end-systolic volume, SV stroke volume, EF ejection fraction, BSA body surface area

a Pooled weighted values from references $[18,23,24]$

${ }^{b}$ Pooled weighted values from references $[18,23]$

${ }^{c}$ Calculated as mean ${ }_{\mathrm{p}} \pm 2 * \mathrm{SD}_{\mathrm{p}}$

Table 12 Right ventricular parameters for adult women by age group, papillary muscles included in right ventricular volume

\begin{tabular}{|c|c|c|c|c|c|c|c|c|}
\hline \multirow[t]{2}{*}{ Parameter } & \multicolumn{2}{|c|}{ 20-29 years } & \multicolumn{2}{|c|}{ 30-39 years } & \multicolumn{2}{|c|}{ 40-49 years } & \multicolumn{2}{|c|}{$50-59$ years } \\
\hline & $\mathbf{n}$ & $\operatorname{Mean}_{\mathrm{p}} \pm \mathrm{SD}_{\mathrm{p}}(\mathrm{LL}-\mathrm{UL})^{\mathrm{c}}$ & $\mathbf{n}$ & $\operatorname{Mean}_{\mathrm{p}} \pm \mathrm{SD}_{\mathrm{p}}(\mathrm{LL}-\mathrm{UL})^{\mathrm{c}}$ & $\mathbf{n}$ & $\operatorname{Mean}_{\mathrm{p}} \pm \mathrm{SD}_{\mathrm{p}}(\mathrm{LL}-\mathrm{UL})^{\mathrm{c}}$ & $\mathrm{n}$ & $\operatorname{Mean}_{\mathrm{p}} \pm \mathrm{SD}_{\mathrm{p}}(\mathrm{LL}-\mathrm{UL})^{*}$ \\
\hline RVEDV/BSA $\left(\mathrm{ml} / \mathrm{m}^{2}\right)^{\mathrm{a}}$ & 47 & $78 \pm 12(55-101)$ & 51 & $76 \pm 12(51-100)$ & 46 & $74 \pm 14(46-102)$ & 46 & $69 \pm 13(42-95)$ \\
\hline RVESV/BSA $\left(\mathrm{ml} / \mathrm{m}^{2}\right)^{\mathrm{a}}$ & 47 & $33 \pm 12(10-56)$ & 51 & $31 \pm 8(15-48)$ & 46 & $29 \pm 8(13-45)$ & 46 & $28 \pm 8(11-44)$ \\
\hline $\mathrm{RVSV} / \mathrm{BSA}\left(\mathrm{ml} / \mathrm{m}^{2}\right)^{\mathrm{b}}$ & 37 & $46 \pm 9(28-63)$ & 33 & $45 \pm 12(22-69)$ & 35 & $47 \pm 11(24-69)$ & 37 & $42 \pm 10(22-62)$ \\
\hline $\operatorname{RVEF}(\%)^{\mathrm{a}}$ & 47 & $56 \pm 11(34-78)$ & 51 & $58 \pm 9(39-77)$ & 46 & $60 \pm 8(44-76)$ & 46 & $61 \pm 8(44-78)$ \\
\hline
\end{tabular}

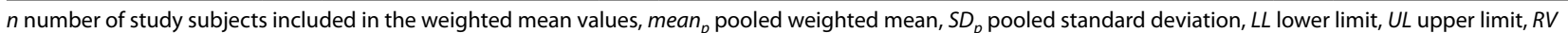
right ventricular, EDV end-diastolic volume, ESV end-systolic volume, SV stroke volume, EF ejection fraction, BSA body surface area

a Pooled weighted values from references $[18,23,24]$

${ }^{b}$ Pooled weighted values from references $[18,23]$

${ }^{c}$ Calculated as mean $\mathrm{p}_{\mathrm{p}} \pm 2 * \mathrm{SD}_{\mathrm{p}}$
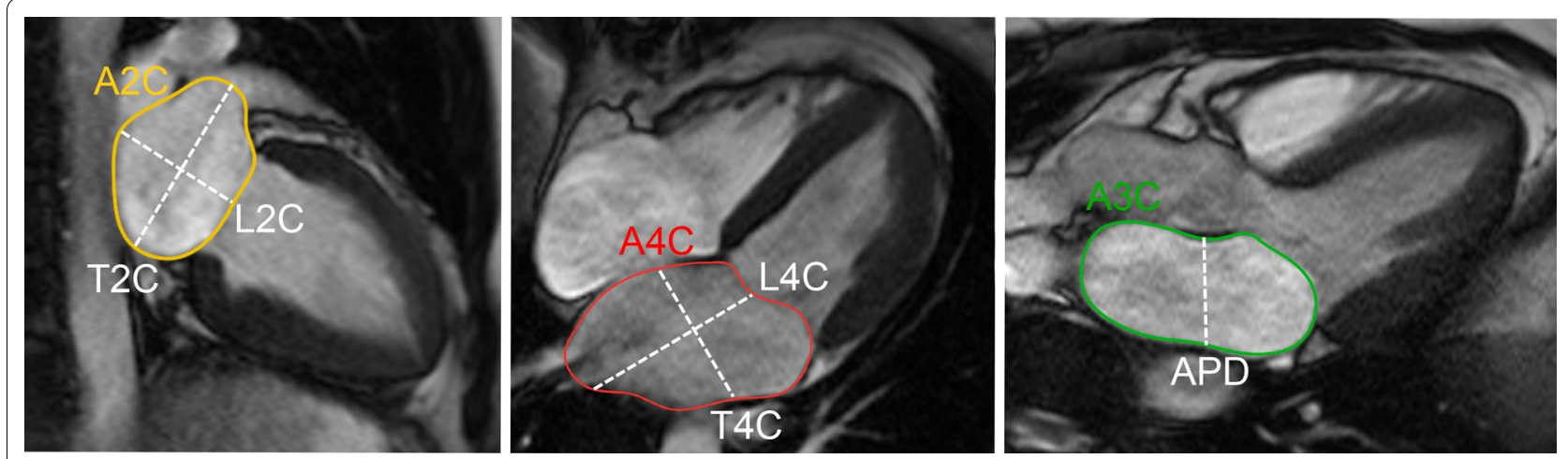

Fig. 3 Measurement of left atrial area (A2Ch, A4Ch, A3C), longitudinal (L2Ch, L4Ch), transverse (T2Ch, T4Ch) and anteroposterior (APD) diameters on the 2-, 4- and 3-chamber views according to reference [31]

\section{Demographic parameters}

Body surface area (BSA) has been shown to have a significant independent influence on LA volume and most diameters [31]. Per Sievers et al. [35], age was not an independent predictor of LA maximal volume or diameter in normal individuals. Men have a larger maximal LA volume compared to women [31, 35].

\section{Studies included in this review}

There are nine publications for reference values of the adult LA (volume and/or diameter and/or area) based 
Table 13 References, normal left atrial volumes, function and dimensions in the adult

\begin{tabular}{|c|c|c|c|}
\hline First author, year & CMR technique & n, male:female & Age range (years) \\
\hline Sievers, 2005 [35] & $1.5 \mathrm{~T}, 2,3$ and 4 chamber bSSFP; measurement of diameters & $59: 52$ & $25-73$ \\
\hline Maceira, 2010 [31] & $\begin{array}{l}1.5 \mathrm{~T} \text {, short axis, } 2,3 \text { and } 4 \text { chamber bSSFP; } 3 \mathrm{D} \text { modeling and measurement of area and } \\
\text { diameters; atrial appendage included, pulmonary veins excluded (for volume analysis) }\end{array}$ & $60: 60$ & $20-80$ \\
\hline Le, 2016 [11] & $\begin{array}{l}3 \text { T, } 2 \text { and } 4 \text { chamber bSSFP; quantification of volume; biplane area-length method; atrial } \\
\text { appendage and pulmonary veins excluded }\end{array}$ & $91: 89$ & $20-69$ \\
\hline Le Ven, 2016 [14] & $\begin{array}{l}1.5 \mathrm{~T} \text {, short axis bSSFP; quantification of volume and function; Simpson's method; atrial } \\
\text { appendage included, pulmonary veins excluded }\end{array}$ & 195:239 & $18-36$ \\
\hline Aquaro, 2017 [18] & 1.5 T, 4 chamber bSSFP; measurement of area & 173:135 & $16->60$ \\
\hline $\mathrm{Li}, 2017$ [33] & $\begin{array}{l}3 \text { T, short axis, 2, } 3 \text { and } 4 \text { chamber bSSFP; measurement of volume, function (biplane area- } \\
\text { length and Simpson's method atrial appendage excluded) and diameter }\end{array}$ & $66: 69$ & $23-83$ \\
\hline Petersen, 2017 [16] & $\begin{array}{l}1.5 \text { T, } 2 \text { and } 4 \text { chamber bSSFP; quantification of volume and function; biplane area-length } \\
\text { method; atrial appendage and pulmonary veins excluded }\end{array}$ & $371: 433$ & $45-74$ \\
\hline Zemrak, 2017 [34] & $\begin{array}{l}1.5 \text { T, } 2 \text { and } 4 \text { chamber bSSFP; quantification of volume; biplane area-length method; atrial } \\
\text { appendage and pulmonary veins excluded }\end{array}$ & 109:174 & $(65 \pm 9)^{a}$ \\
\hline Funk, $2018[32]$ & $\begin{array}{l}1.5 \text { T and } 3 \text { T, } 2 \text { and } 4 \text { chamber bSSFP; quantification of volume and function; biplane area- } \\
\text { length method; atrial appendage and pulmonary veins excluded }\end{array}$ & 105:77 & $19-76$ \\
\hline
\end{tabular}

$n$ number of study subjects, bSSFP balanced steady-state free precession, 3D 3-dimensional

${ }^{a}$ Mean \pm SD (age-range not provided in original publication)

on bSSFP imaging with sufficient sample size $(\mathrm{n}>40)$ and these are reported in Table 13. Four of these publications used the biplane area-length method, one used the Simpson's method, one used both, one used a 3D modeling technique and the remainder measured diameters or areas. Publications reporting population-based cohort data rather than true normal data have been excluded from the current analysis as have publications that incompletely describe the measurement method used [22] or the manner in which pulmonary veins/LA appendage were handled. Normal values for LA volumes and function are presented in Table 14, and normal values for LA diameters in Table 15.

\section{Right atrial dimensions and functions in the adult CMR acquisition parameters}

There is no consensus in the literature regarding acquisition and measurement method for the right atrium (RA). Published methods for RA volume include the modified Simpson's method, the biplane area-length method and 3D-modeling [23, 24, 37]. For Simpson's method and 3D modeling, a stack of cine bSSFP images in the SAx view are analyzed. For the biplane area-length method, a 4-chamber view and a RV 2-chamber view are utilized [33] (Fig. 4).

\section{CMR analysis methods}

The inferior and superior vena cava are excluded from the RA volume but there is variability in the inclusion $[14,37]$ or exclusion [33] of the RA appendage.

The maximal RA volume is achieved during ventricular systole and can be defined as the last cine image before opening of the tricuspid valve. The minimal RA volume can be defined as the first cine image after closure of the tricuspid valve.

\section{Demographic parameters}

Maceira et al. demonstrated the relationship of most RA parameters to BSA, but there was no influence of age on atrial parameters and no influence of gender on atrial volumes [37]. Other studies have demonstrated an influence of gender $[14,33]$ and age $[11,33]$ on some RA parameters. In the study by LeVen et al. gender was independently associated with RA end-diastolic volume and RA end-systolic volume with men having greater values compared to women [14]. In the study by Li et al. the longitudinal RA diameter measured in the 2 chamber and 4 chamber view indexed to BSA and the indexed transverse diameter measured on the 4 chamber view were greater in women than in men [33]. Further, the RA volume indexed to BSA was larger in males than in females [33]. Le et al. found a week correlation between the RA area indexed to BSA with age [11].

\section{Studies included in this review}

There are five publications with reference values for the RA based on bSSFP imaging with sufficient sample size to be included [11, 14, 18, 33, 37] (Table 16). Pooled weighted mean values for RA volumes and function are provided in Table 17 using the biplane area-length method (RA appendage excluded) or Simpson's method (either RA appendage included or excluded) for men and women. Pooled weighted mean values for RA areas and diameters are provided in Table 18 for men and women. 
Table 14 Left atrial volumes and function in the adult for men and women, SSFP technique

\begin{tabular}{|c|c|c|c|c|c|c|c|c|c|}
\hline \multirow[t]{2}{*}{ Method } & \multirow[t]{2}{*}{ Parameter } & \multicolumn{4}{|c|}{ Men } & \multicolumn{4}{|c|}{ Women } \\
\hline & & $\mathbf{n}$ & Mean $_{p}$ & $S D_{p}$ & LL-ULj & n & Mean $_{p}$ & $\mathrm{SD}_{\mathrm{p}}$ & LL-UL \\
\hline \multirow[t]{7}{*}{ Biplane area-length method; LA appendage excluded } & Max. LA volume $(\mathrm{ml})^{\mathrm{a}}$ & 734 & 72 & 20 & $31-112$ & 841 & 64 & 18 & $28-100$ \\
\hline & Max. LA volume/BSA $\left(\mathrm{ml} / \mathrm{m}^{2}\right)^{\mathrm{a}}$ & 734 & 38 & 11 & $17-59$ & 841 & 39 & 11 & $17-61$ \\
\hline & Min. LA volume $(\mathrm{ml})^{\mathrm{b}}$ & 171 & 25 & 10 & $6-44$ & 146 & 22 & 8 & $7-38$ \\
\hline & Min. LA volume/BSA $\left(\mathrm{ml} / \mathrm{m}^{2}\right)^{c}$ & 171 & 14 & 5 & $3-24$ & 146 & 13 & 5 & $4-23$ \\
\hline & LA stroke volume $(\mathrm{ml})^{\mathrm{d}}$ & 468 & 44 & 12 & $21-67$ & 509 & 42 & 10 & $21-62$ \\
\hline & LA stroke volume/BSA $\left(\mathrm{ml} / \mathrm{m}^{2}\right)^{\mathrm{e}}$ & 363 & 22 & 6 & $10-34$ & 432 & 22 & 6 & $10-34$ \\
\hline & LA ejection fraction $(\%)^{f}$ & 534 & 62 & 8 & $46-77$ & 578 & 63 & 8 & $48-78$ \\
\hline \multirow[t]{5}{*}{ Simpson's method; LA appendage excluded } & Max. LA volume $(\mathrm{ml})^{9}$ & 66 & 70 & 15 & 40-99 & 69 & 66 & 13 & 39-93 \\
\hline & Max. LA volume/BSA $\left(\mathrm{ml} / \mathrm{m}^{2}\right)^{\mathrm{g}}$ & 66 & 41 & 8 & $24-57$ & 69 & 44 & 8 & $28-60$ \\
\hline & Min. LA volume $(\mathrm{ml})^{9}$ & 66 & 32 & 9 & $15-50$ & 69 & 28 & 7 & $15-42$ \\
\hline & Min. LA volume/BSA $\left(\mathrm{ml} / \mathrm{m}^{2}\right)^{g}$ & 66 & 19 & 5 & $9-28$ & 69 & 19 & 4 & $11-27$ \\
\hline & LA ejection fraction (\%) ${ }^{g}$ & 66 & 54 & 8 & $38-70$ & 69 & 57 & 6 & $45-69$ \\
\hline \multirow[t]{7}{*}{ Simpson's method; LA appendage included } & Max. LA volume $(\mathrm{ml})^{\mathrm{h}}$ & 256 & 78 & 18 & $42-115$ & 298 & 66 & 14 & $37-94$ \\
\hline & Max. LA volume/BSA $\left(\mathrm{ml} / \mathrm{m}^{2}\right)^{\mathrm{h}}$ & 256 & 40 & 8 & $25-56$ & 298 & 39 & 7 & $25-53$ \\
\hline & Min. LA volume $(\mathrm{ml})^{i}$ & 196 & 32 & 9 & $14-50$ & 238 & 24 & 7 & $10-38$ \\
\hline & Min. LA volume/BSA $\left(\mathrm{ml} / \mathrm{m}^{2}\right)^{\mathrm{i}}$ & 196 & 17 & 4 & $9-25$ & 238 & 15 & 4 & $7-23$ \\
\hline & LA stroke volume $(\mathrm{ml})^{\mathrm{i}}$ & 196 & 47 & 13 & $21-73$ & 238 & 39 & 10 & $19-59$ \\
\hline & LA stroke volume/BSA $\left(\mathrm{ml} / \mathrm{m}^{2}\right)^{\mathrm{i}}$ & 196 & 24 & 6 & $12-36$ & 238 & 24 & 5 & $14-34$ \\
\hline & LA ejection fraction (\%) ${ }^{i}$ & 196 & 59 & 8 & $43-75$ & 238 & 61 & 7 & $47-75$ \\
\hline
\end{tabular}

$n$ number of study subjects included in the weighted mean values, $b S S F P$ balanced steady-state free precession, mean ${ }_{p}$ pooled weighted mean, $S D_{p}$ pooled standard deviation, LL lower limit, UL upper limit, Max. maximal, Min. minimal, LA left atrial, BSA body surface area

a Pooled weighted values from references $[11,16,32-34]$

${ }^{\mathrm{b}}$ Pooled weighted values from references $[22,32,33]$

' Pooled weighted values from references [32, 33]

d Pooled weighted values from references [16, 32]

e Values from reference [16]

${ }^{f}$ Pooled weighted values from references $[16,22,32,33]$

${ }^{g}$ Values from reference [33]

h Pooled weighted values from references [14, 31]

iValues from reference [14]

j Calculated as mean $_{\mathrm{p}} \pm 2 * \mathrm{SD}_{\mathrm{p}}$

\section{Additional RA function parameters}

Reference ranges for parameters characterizing RA function, including the reservoir, conduit and pump function, can be found in a separate publication by Maceira et al. [38].

\section{Left and right ventricular dimensions and function in children}

The presentation of normal values in children is different than in the adult population due to continuous changes in body weight and height as a function of age. Normal data in children are frequently presented in percentiles and/or $\mathrm{Z}$-scores (standard deviation score). Z-scores are given as
Even though previous studies [39-41] have reported a linear correlation between ventricular volumes and BSA in children, there is increasing evidence that the assumption of a simple linear or exponential relationship between somatic growth and age may not be correct. Moreover the relationship between cardiac growth and body growth is still not clearly understood and may vary along age in the developing child [42, 43].

The construction of reference curves using the Lamda-Mu-Sigma (LMS) method is a different way of creating normalized growth percentile curves. In this approach after a power transformation skewness of

$$
\mathrm{z}-\text { value }=(\text { measurement }- \text { mean of the population }) /(\text { standard deviation of the mean of the population }) \text {. }
$$


Table 15 Left atrial diameter and area in the adult for men and women, bSSFP technique

\begin{tabular}{|c|c|c|c|c|c|c|c|c|}
\hline \multirow[t]{2}{*}{ Parameter } & \multicolumn{4}{|c|}{ Men } & \multicolumn{4}{|c|}{ Women } \\
\hline & $n$ & Mean $_{p}$ & $S D_{p}$ & $L L-U L^{f}$ & $\bar{n}$ & Mean $_{p}$ & $S D_{p}$ & $L L-U L^{f}$ \\
\hline Max. LA area $2 \mathrm{Ch}\left(\mathrm{cm}^{2}\right)^{\mathrm{a}}$ & 60 & 21 & 5 & $12-30$ & 60 & 19 & 5 & $10-28$ \\
\hline Max. LA area $2 \mathrm{Ch} / \mathrm{BSA}\left(\mathrm{cm}^{2} / \mathrm{m}^{2}\right)^{\mathrm{a}}$ & 60 & 11 & 2 & $6-16$ & 60 & 11 & 2 & $6-16$ \\
\hline Max. LA area $3 \mathrm{Ch}\left(\mathrm{cm}^{2}\right)^{\mathrm{a}}$ & 60 & 19 & 4 & $12-26$ & 60 & 17 & 4 & $10-24$ \\
\hline Max. LA area 3Ch/BSA $\left(\mathrm{cm}^{2} / \mathrm{m}^{2}\right)^{\mathrm{a}}$ & 60 & 10 & 2 & $6-14$ & 60 & 10 & 2 & $6-14$ \\
\hline Max. LA area $4 \mathrm{Ch}\left(\mathrm{cm}^{2}\right)^{\mathrm{b}}$ & 233 & 23 & 5 & $13-32$ & 173 & 21 & 4 & $13-29$ \\
\hline Max. LA area $4 \mathrm{Ch} / \mathrm{BSA}\left(\mathrm{cm}^{2} / \mathrm{m}^{2}\right)^{\mathrm{b}}$ & 233 & 12 & 2 & $7-16$ & 195 & 12 & 2 & $8-15$ \\
\hline Max. LA longitudinal diameter $2 \mathrm{Ch}(\mathrm{cm})^{c}$ & 185 & 4.9 & 0.7 & $3.5-6.2$ & 181 & 4.6 & 0.7 & $3.3-5.9$ \\
\hline Max. LA longitudinal diameter $2 \mathrm{Ch} / \mathrm{BSA}\left(\mathrm{cm} / \mathrm{m}^{2}\right)^{c}$ & 185 & 2.6 & 0.5 & $1.6-3.6$ & 181 & 2.8 & 0.6 & $1.6-3.9$ \\
\hline Max. LA transverse diameter $2 \mathrm{Ch}(\mathrm{cm})^{d}$ & 126 & 4.4 & 0.6 & $3.2-5.6$ & 129 & 4.3 & 0.5 & $3.3-5.2$ \\
\hline Max. LA transverse diameter $2 \mathrm{Ch} / \mathrm{BSA}\left(\mathrm{cm} / \mathrm{m}^{2}\right)^{\mathrm{d}}$ & 126 & 2.4 & 0.3 & $1.7-3.0$ & 129 & 2.7 & 0.3 & $2.2-3.2$ \\
\hline Max. LA longitudinal diameter $3 \mathrm{Ch}(\mathrm{cm})^{\mathrm{e}}$ & 66 & 5.5 & 0.6 & $4.2-6.8$ & 69 & 5.4 & 0.7 & $4.0-6.7$ \\
\hline Max. LA longitudinal diameter 3Ch/BSA $\left(\mathrm{cm} / \mathrm{m}^{2}\right)^{\mathrm{e}}$ & 66 & 3.2 & 0.4 & $2.4-4.0$ & 69 & 3.6 & 0.5 & $2.7-4.6$ \\
\hline Max. LA antero-posterior diameter $3 \mathrm{Ch}(\mathrm{cm})^{c}$ & 185 & 3.0 & 0.5 & $2.0-4.0$ & 181 & 3.0 & 0.5 & $2.0-4.0$ \\
\hline Max. LA antero-posterior diameter $3 \mathrm{Ch} / \mathrm{BSA}\left(\mathrm{cm} / \mathrm{m}^{2}\right)^{\mathrm{c}}$ & 185 & 1.6 & 0.3 & $1.0-2.2$ & 181 & 1.8 & 0.4 & $1.1-2.5$ \\
\hline Max. LA longitudinal diameter 4Ch $(\mathrm{cm})^{d}$ & 126 & 5.8 & 0.6 & $4.6-7.1$ & 129 & 5.5 & 0.6 & $4.2-6.8$ \\
\hline Max. LA longitudinal diameter $4 \mathrm{Ch} / \mathrm{BSA}\left(\mathrm{cm} / \mathrm{m}^{2}\right)^{d}$ & 126 & 3.2 & 0.4 & $2.3-4.1$ & 129 & 3.5 & 0.5 & $2.5-4.4$ \\
\hline Max. LA transverse diameter $4 \mathrm{Ch}(\mathrm{cm})^{c}$ & 185 & 4.3 & 0.5 & $3.3-5.3$ & 181 & 4.1 & 0.5 & $3.1-5.1$ \\
\hline Max. LA transverse diameter $4 \mathrm{Ch} / \mathrm{BSA}\left(\mathrm{cm} / \mathrm{m}^{2}\right)^{\mathrm{c}}$ & 185 & 2.2 & 0.3 & $1.6-2.9$ & 181 & 2.5 & 0.4 & $1.8-3.2$ \\
\hline \multicolumn{9}{|c|}{$\begin{array}{l}n \text { number of study subjects included in the weighted mean values, } b S S F P \text { balanced steady-state free precession, mean } p_{p} \text { pooled weighted mean, } S D_{p} \text { pooled standard } \\
\text { deviation, } L L \text { lower limit, UL upper limit, Max. maximal, LA left atrial, } 2 C h 2 \text { chamber view, } 3 C h \text { chamber view, } 4 C h 4 \text { chamber view, BSA body surface area }\end{array}$} \\
\hline \multicolumn{9}{|l|}{${ }^{a}$ Values from reference [31] } \\
\hline \multicolumn{9}{|l|}{${ }^{\mathrm{b}}$ Pooled weighted values from references $[18,31]$} \\
\hline \multicolumn{9}{|l|}{ ' Pooled weighted values from references $[31,33,35]$} \\
\hline \multicolumn{9}{|l|}{ d Pooled weighted values from references $[31,33]$} \\
\hline \multicolumn{9}{|l|}{ e Values from reference [33] } \\
\hline${ }^{f}$ Calculated as mean ${ }_{p} \pm 2 * \mathrm{SD}_{p}$ & & & & & & & & \\
\hline
\end{tabular}

the data can be transformed into normality and trends are summarized in a smooth curve (L); trends in the mean (M) and coefficient of variation (S) are similarly smoothed. LMS curves are easy to use in daily practice and can account for nonlinear relationships between body and cardiac size and age.

The LMS method is highly efficient to obtain normality in small datasets, for instance in the group of young children. Thus, even extreme values (small children) can be so converted into exact standard deviation scores [44].

\section{Demographic parameters}

The largest cohort of normal data on ventricular size and function in paediatric patients using the bSSFP sequence refers to a population of 141 healthy children collected in three European reference centers. All subjects were Caucasian and included 68 boys and 73 girls. Age distribution, body size and heart rate were equal between genders. Only 12/141 children were younger than 6 years [45].

Boys had larger ventricles than girls [45]. LVEF was found to be slightly higher in boys (67\% vs 65\%; p 0.01), but not for the RV [45]. Gender differences are more marked in older children, indicating that gender is more important after puberty and in adulthood.

\section{Studies included in this review}

Table 19 shows studies meeting inclusion criteria. The reference values for the $L V$ and $R V$ presented in the study by van der Ven [45] have been pooled from three previous studies [39-41], that have been reported separately in the previous version of our review [1]. Data are presented in percentile curves referred to age by using the LMS Method (Figs. 5, 6).

\section{CMR analysis methods}

For calculation of reference values from reference [45], the original bSSFP images (short axis) have been re-analysed by manual segmentation by one operator, after consensus on the segmentation rules was established within the group. These followed the standards proposed by SCMR [46], except for the trabeculations of the RV, required for calculating the RV mass. In the $\mathrm{RV}$ major trabeculae were included in the myocardium 

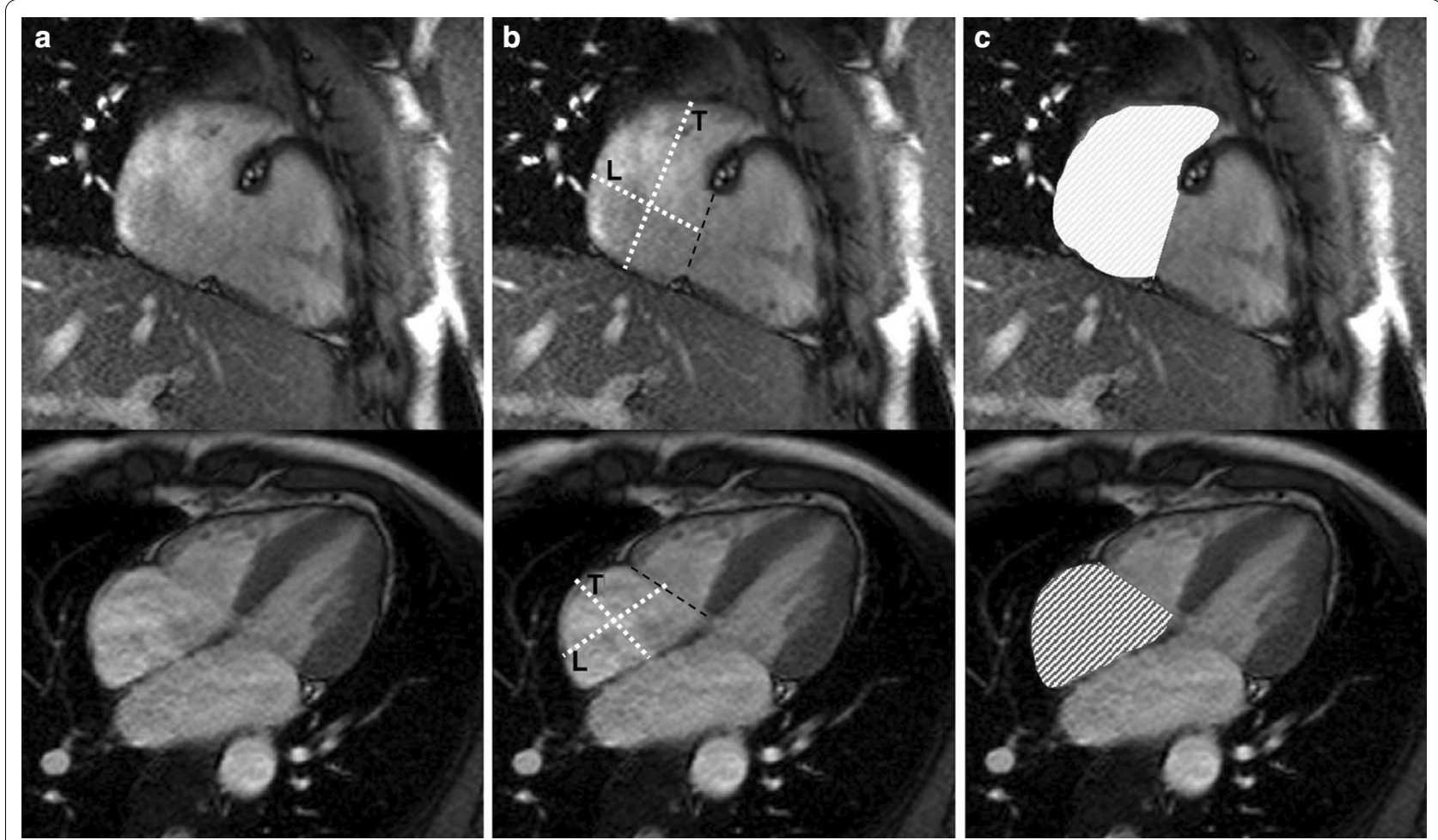

Fig. 4 Measurement of right atrial (RA) parameters according to [37]. Areas and diameters were measured in atrial diastole (maximal size of the left atrium) on the 2-chamber (top row) and 4-chamber (bottom row) views. In B), longitudinal diameter (L) is obtained from the posterior wall of the RA to the center of the tricuspid plane, and transverse diameter $(T)$ is obtained perpendicular to the longitudinal diameter, at the mid level of the RA. C shows measurements of the area for both views including the RA appendage

Table 16 References, normal right atrial volumes, function and dimensions in the adult

\begin{tabular}{llcc}
\hline First author, year & CMR technique & n, male:female & Age range (years) \\
\hline Maceira, 2013 [37] & $\begin{array}{l}\text { 1.5 T, short axis, RV 2 chamber and 4 chamber bSSFP, 3D modeling and measurement of } \\
\text { area and diameters, atrial appendage included for volume analysis }\end{array}$ & $60: 60$ & $20-80$ \\
Le Ven, 2015 [14] & $\begin{array}{l}\text { 1.5 T, short axis bSSFP, quantification of volume and function (Simpson's method), atrial } \\
\text { appendage included }\end{array}$ & $196: 238$ & $25-73$ \\
Le, 2016 [11] & 3.0 T, 4 chamber bSSFP, measurement of area & $91: 89$ & $20-69$ \\
Aquaro, 2017 [18] & 1.5 T, 4 chamber bSSFP, measurement of area & $173: 135$ & $16->60$ \\
Li, 2017 [33] & $\begin{array}{l}\text { 3.0 T, Short axis, RV 2 chamber and 4 chamber bSSFP, measurement of diameter, volume } \\
\text { and function (biplane area-length and Simpson's method), atrial appendage excluded }\end{array}$ & $66: 69$ & $23-83$
\end{tabular}

$n$ number of study subject, $b S S F P$ balanced steady-state free precession, $R V$ right ventricular

if they were visualized as being connected to the RV wall in more than 2 adjacent slices. Trabecular islands not connected to the wall were included in the blood pool [45].

\section{Left and right atrial dimensions and function in children \\ CMR acquisition parameters}

LA and RA dimensions and function were evaluated using bSSFP technique in a single publication [47],
(Table 19). Measurements were obtained on a stack of transverse cine bSSFP images with a slice thickness between 5 and $6 \mathrm{~mm}$ without interslice gap [47].

\section{CMR analysis methods}

In [47], the pulmonary veins, the superior and inferior vena cava and the coronary sinus were excluded from the LA and RA volume, respectively, while the atrial appendages were included in the volume of the respective 
Table 17 Right atrial volumes and function in the adult for men and women

\begin{tabular}{|c|c|c|c|c|c|c|c|c|c|}
\hline \multirow[t]{2}{*}{ Method } & \multirow[t]{2}{*}{ Parameter } & \multicolumn{4}{|c|}{ Men } & \multicolumn{4}{|c|}{ Women } \\
\hline & & $\mathrm{N}$ & Mean $_{p}$ & $S D_{p}$ & ${\mathrm{LL}-U L^{d}}^{\mathrm{d}}$ & $n$ & Mean $_{p}$ & $S D_{p}$ & LL-UL ${ }^{d}$ \\
\hline \multirow[t]{5}{*}{ Biplane area-length method; RA appendage excluded } & Max. RA volume $(\mathrm{ml})^{\mathrm{a}}$ & 66 & 65 & 20 & 24-105 & 69 & 53 & 14 & $24-81$ \\
\hline & Max. RA volume/BSA $\left(\mathrm{ml} / \mathrm{m}^{2}\right)^{\mathrm{a}}$ & 66 & 38 & 12 & $15-61$ & 69 & 35 & 10 & $16-54$ \\
\hline & Min. RA volume $(\mathrm{ml})^{\mathrm{a}}$ & 66 & 32 & 12 & $9-55$ & 69 & 23 & 7 & $9-37$ \\
\hline & Min. RA volume/BSA $\left(\mathrm{ml} / \mathrm{m}^{2}\right)^{\mathrm{a}}$ & 66 & 19 & 7 & $5-32$ & 69 & 15 & 5 & $6-24$ \\
\hline & RA ejection fraction (\%) ${ }^{\mathrm{a}}$ & 66 & 50 & 9 & $32-68$ & 69 & 56 & 9 & $38-74$ \\
\hline \multirow[t]{5}{*}{ Simpson's method; RA appendage excluded } & Max. RA volume $(\mathrm{ml})^{\mathrm{a}}$ & 66 & 89 & 22 & $46-132$ & 69 & 77 & 16 & $45-108$ \\
\hline & Max. RA volume/BSA $\left(\mathrm{ml} / \mathrm{m}^{2}\right)^{\mathrm{a}}$ & 66 & 52 & 12 & $28-76$ & 69 & 51 & 10 & $31-71$ \\
\hline & Min. RA volume $(\mathrm{ml})^{\mathrm{a}}$ & 66 & 46 & 16 & $14-79$ & 69 & 35 & 9 & $17-53$ \\
\hline & Min. RA volume/BSA $\left(\mathrm{ml} / \mathrm{m}^{2}\right)^{\mathrm{a}}$ & 66 & 27 & 9 & $9-45$ & 69 & 23 & 6 & $12-35$ \\
\hline & RA ejection fraction (\%) ${ }^{\mathrm{a}}$ & 66 & 49 & 10 & $29-69$ & 69 & 54 & 9 & $36-72$ \\
\hline \multirow[t]{7}{*}{ Simpson's method; RA appendage included } & Max. RA volume $(\mathrm{ml})^{\mathrm{b}}$ & 256 & 108 & 25 & 59-158 & 298 & 85 & 18 & $49-122$ \\
\hline & Max. RA volume/BSA $\left(\mathrm{ml} / \mathrm{m}^{2}\right)^{\mathrm{b}}$ & 256 & 56 & 12 & $32-79$ & 298 & 50 & 10 & $31-69$ \\
\hline & Min. RA volume $(\mathrm{ml})^{c}$ & 196 & 50 & 17 & $16-84$ & 238 & 33 & 11 & $11-55$ \\
\hline & Min. RA volume/BSA $\left(\mathrm{ml} / \mathrm{m}^{2}\right)^{c}$ & 196 & 26 & 8 & $10-42$ & 238 & 20 & 6 & $8-32$ \\
\hline & RA stroke volume $(\mathrm{ml})^{c}$ & 196 & 58 & 16 & $26-90$ & 238 & 47 & 12 & $23-71$ \\
\hline & RA stroke volume/BSA $\left(\mathrm{ml} / \mathrm{m}^{2}\right)^{\mathrm{c}}$ & 196 & 30 & 8 & $14-46$ & 238 & 28 & 7 & $14-42$ \\
\hline & RA ejection fraction $(\%)^{c}$ & 196 & 54 & 10 & $34-74$ & 238 & 59 & 9 & $41-77$ \\
\hline
\end{tabular}

$n$ number of study subjects included in the weighted mean values, mean $p$ pooled weighted mean, $S D_{p}$ pooled standard deviation, $L L$ lower limit, UL upper limit, Max. maximal, Min. minimal, $R A$ right atrial, $B S A$ body surface area

${ }^{\text {a }}$ Values from reference [33]

${ }^{b}$ Pooled weighted values from references $[14,37]$

'Values from reference [14]

${ }^{\mathrm{d}}$ Calculated as mean $_{\mathrm{p}} \pm 2 * \mathrm{SD}_{\mathrm{p}}$

Table 18 Right atrial diameter and area in the adult for men and women, bSSFP technique

\begin{tabular}{|c|c|c|c|c|c|c|c|c|}
\hline \multirow[t]{2}{*}{ Parameter } & \multicolumn{4}{|c|}{ Men } & \multicolumn{4}{|c|}{ Women } \\
\hline & $n$ & Mean $_{p}$ & $S D_{p}$ & $\overline{L L-U L^{d}}$ & $n$ & $\operatorname{Mean}_{p}$ & $S D_{p}$ & LL-UL ${ }^{d}$ \\
\hline Max. RA area $2 \mathrm{Ch}\left(\mathrm{cm}^{2}\right)^{\mathrm{a}}$ & 60 & 23 & 4 & $15-31$ & 60 & 21 & 4 & $13-29$ \\
\hline Max. RA area $2 \mathrm{Ch} / \mathrm{BSA}\left(\mathrm{cm}^{2} / \mathrm{m}^{2}\right)^{\mathrm{a}}$ & 60 & 12 & 2 & $7-17$ & 60 & 12 & 2 & $7-17$ \\
\hline Max. RA area $4 \mathrm{Ch}\left(\mathrm{cm}^{2}\right)^{\mathrm{b}}$ & 324 & 21 & 4 & $13-30$ & 284 & 19 & 3 & $12-26$ \\
\hline Max. RA area $4 \mathrm{Ch} / \mathrm{BSA}\left(\mathrm{cm}^{2} / \mathrm{m}^{2}\right)^{b}$ & 324 & 11 & 2 & $7-15$ & 284 & 12 & 2 & $8-15$ \\
\hline Max. RA longitudinal diameter $2 \mathrm{Ch}(\mathrm{cm})^{c}$ & 126 & 5.5 & 0.6 & $4.2-6.7$ & 129 & 5.1 & 0.6 & $3.9-6.3$ \\
\hline Max. RA longitudinal diameter $2 \mathrm{Ch} / \mathrm{BSA}\left(\mathrm{cm} / \mathrm{m}^{2}\right)^{c}$ & 126 & 3.0 & 0.4 & $2.3-3.7$ & 129 & 3.2 & 0.4 & $2.3-4.1$ \\
\hline Max. RA transverse diameter $2 \mathrm{Ch}(\mathrm{cm})^{c}$ & 126 & 4.2 & 0.9 & $2.4-6.0$ & 129 & 4.1 & 0.9 & $2.4-5.9$ \\
\hline Max. RA transverse diameter $2 \mathrm{Ch} / \mathrm{BSA}\left(\mathrm{cm} / \mathrm{m}^{2}\right)^{c}$ & 126 & 2.3 & 0.5 & $1.3-3.3$ & 129 & 2.6 & 0.6 & $1.5-3.7$ \\
\hline Max. RA longitudinal diameter $4 \mathrm{Ch}(\mathrm{cm})^{c}$ & 126 & 5.3 & 0.6 & $4.0-6.6$ & 129 & 5.1 & 0.6 & $4.0-6.3$ \\
\hline Max. RA longitudinal diameter 4Ch/BSA $\left(\mathrm{cm} / \mathrm{m}^{2}\right)^{c}$ & 126 & 2.9 & 0.4 & $2.2-3.7$ & 129 & 3.2 & 0.4 & $2.4-4.0$ \\
\hline Max. RA transverse diameter $4 \mathrm{Ch}(\mathrm{cm})^{c}$ & 126 & 4.8 & 0.6 & $3.7-5.9$ & 129 & 4.3 & 0.6 & $3.2-5.4$ \\
\hline Max. RA transverse diameter $4 \mathrm{Ch} / \mathrm{BSA}\left(\mathrm{cm} / \mathrm{m}^{2}\right)^{c}$ & 126 & 2.6 & 0.3 & $2.1-3.2$ & 129 & 2.7 & 0.3 & $2.0-3.4$ \\
\hline
\end{tabular}

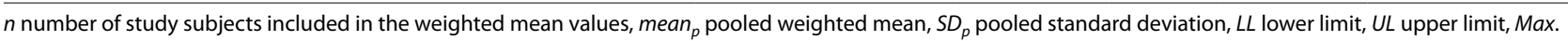
maximal, $R A$ right atrial, $2 C h 2$ chamber view, $3 C h 3$ chamber view, $4 C h 4$ chamber view, BSA body surface area

${ }^{a}$ Values from reference [37]

${ }^{b}$ Pooled weighted values from references $[11,18,37]$

' Pooled weighted values from references $[33,37]$

${ }^{d}$ Calculated as mean m $\pm 2 * \mathrm{SD}_{\mathrm{p}}$ 
Table 19 References, normal dimensions of cardiac chambers in children

\begin{tabular}{|c|c|c|c|}
\hline First author, year & CMR technique & $\mathrm{n}$, male:female & Age range (years) \\
\hline van der Ven, 2019 [45] & $\begin{array}{l}\text { 1.5 T, short axis bSSFP; dimensions of LV and RV; papillary mus- } \\
\text { cles included in LV mass; RV mass measured at end-systole, } \\
\text { major trabeculae included in RV mass when connected to the } \\
\text { ventricular wall, trabeculae not connected to the wall included } \\
\text { in RV volume }\end{array}$ & $68: 73$ & $<1-18$ \\
\hline Sarikouch, 2011 [47] & $\begin{array}{l}1.5 \mathrm{~T} \text {, axial bSSFP; pulmonary veins, superior and inferior vena } \\
\text { cava and coronary sinus excluded, atrial appendages included } \\
\text { from/in left and right atrial volume, respectively }\end{array}$ & $56: 59$ & $4-20$ \\
\hline
\end{tabular}

$n$ number of study subjects, $b S S F P$ balanced steady-state free precession, $L V$ left ventricular, $R V$ right ventricular

atrium. The maximal atrial volume was measured at ventricular end-systole and the minimal atrial volume at ventricular end-diastole.

\section{Demographic parameters}

LA and RA volumes show an increase with age with a plateau after the age of 14 for girls only. Absolute and indexed volumes have been shown to be significantly greater for boys compared to girls (except for the indexed maximal volumes for both atria) [47].

\section{Studies included in this review}

Sarikouch et al. evaluated atrial parameters of 115 healthy children (Table 19) [47] using bSSFP imaging. Data is presented as $L, M, S$ values to enable calculation of the standard deviation score and in percentiles (Tables 20, 21).

\section{Cardiac chamber size in the athlete CMR analysis methods}

Methodologic considerations for CMR analysis are the same as for the non-athletes heart as described in the sections above. In both studies included in this review, papillary muscles and trabeculations were included in the ventricular volumes and excluded from LV and RV mass.

\section{Demographic parameters}

Following the Mitchell classification, sports can be characterized as being high or low in dynamic (endurance, isotonic) versus static (strength/resistance, isometric) training and performance components [48]. Athletic competition can therefore be primarily (a) endurance (e.g. long distance running, swimming), (b) combined (e.g. rowers, cyclists) or (c) strength (e.g. body building and weight training). There are insufficient numbers of study subjects available in the literature to establish normative values for the strength category of athletes [49].

Cardiac chamber sizes may vary depending on the extent of exercise and training. One approach to classification is $9-18 \mathrm{~h}$ of training per week (regular athletes) vs $>18 \mathrm{~h}$ training per week (elite athletes) [50]. Adaptive changes to exercise are greater with higher exercise/ training level [49].

Luijkx found a balanced increase of LV and RV chamber volume in relationship in the athlete heart [51]; a large meta-analysis of the literature had a similar conclusion [49]. RV and LV systolic function is commonly characterized by ejection fraction, but this parameter is known to show the most variation between observers. Nevertheless, RVEF and LVEF are $>50 \%$ in reports of the athlete's heart by CMR [48].

The RV chamber volumes are greater in the athletes heart than in normal individuals [51]. The athlete's $\mathrm{RV}$ volumes may exceed CMR criteria for abnormality in arrhythmogenic right ventricular cardiomyopathy (ARVC). However, RVEF is in the normal range of nonathletes even in the athlete heart (i.e.>50\%) whereas RVEF is abnormally low $(\leq 45 \%)$ in ARVC.

\section{Studies included in this review}

After elimination of redundant publications using the same study population and publications with $>40$ athletes, there is one publication with data on the athlete's heart by Prakken et al. (Table 22) [50]. This study was performed at $1.5 \mathrm{~T}$ and has sufficient description of CMR analysis technique to enable comparison (Tables 23, 24). Papillary muscles and trabeculation were included in ventricular volumes and excluded from myocardial mass. The study by Prakken et al. [50] specified levels of training (regular athletes 9-18 h/week; elite athletes $>18 \mathrm{~h}$ per week), both endurance and combined types of athletic participation were included. In contrast, Tahir et al. [52] identified athletes as those competing in triathlons (classified as 'combined' sport activity and training for more than $10 \mathrm{~h}$ per week) without further subcategorization. Although a smaller size cohort, the study by Tahir et al. may also be useful for the interested reader [52].

Finally, one publication [49] presents a meta-analysis of the literature in an attempt to provide reference 

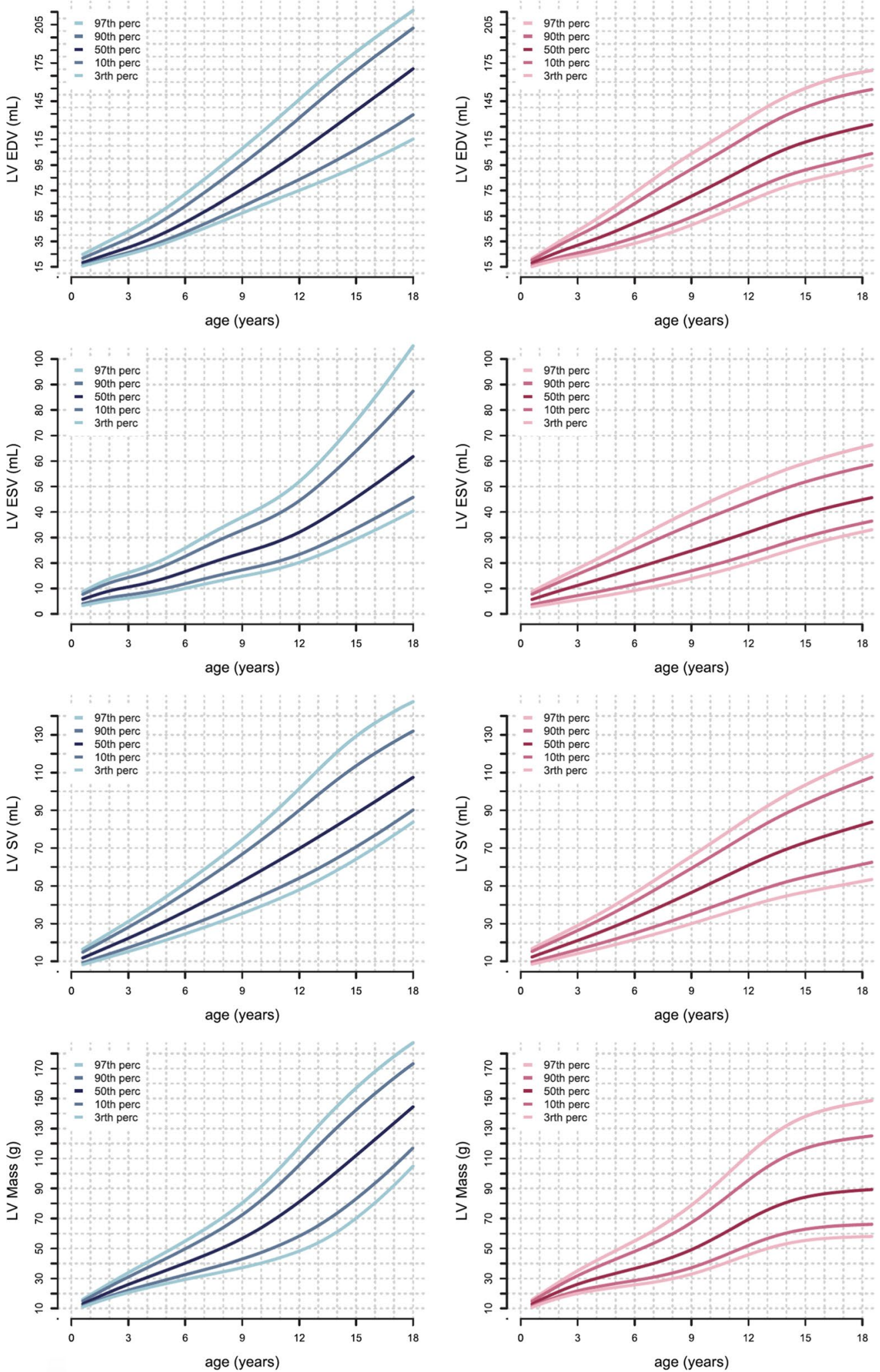

Fig. 5 Reference curves for LV dimensions and function in children, reprinted with permission from reference [45]. Curves for boys are displayed in blue on the left, curves for girls are shown in pink on the right. Reference lines show the 3rd, 10th, 90th and 97th percentile. LV left ventricle, ED end diastolic, ES end systolic, SV stroke volume 

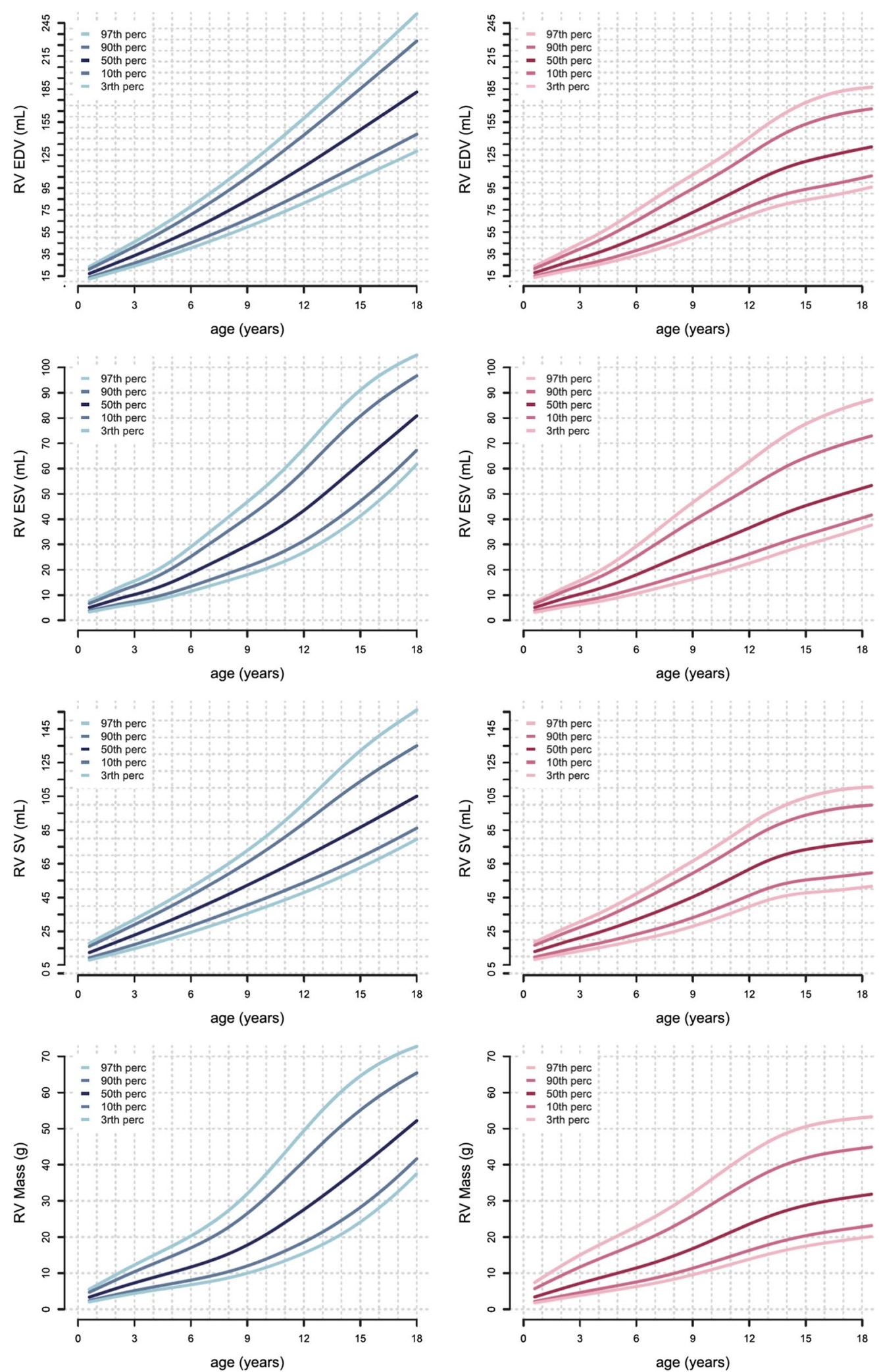

Fig. 6 Reference curves for RV dimensions and function in children, reprinted with permission from reference [45]. Curves for boys are displayed in blue on the left, curves for girls are shown in pink on the right. Reference lines show the 3rd, 10th, 90th and 97th percentile. LV left ventricle, ED end diastolic, ES end systolic, SV stroke volume 
Table 20 Normal left atrial and right atrial volume in boys; LMS parameters to calculate z-scores and percentiles relative to age according to reference [47]

\begin{tabular}{|c|c|c|c|c|c|c|c|c|c|c|c|c|}
\hline \multirow[t]{3}{*}{$\mathrm{Age}^{\mathrm{a}}$} & \multicolumn{6}{|c|}{ Left atrium } & \multicolumn{6}{|c|}{ Right atrium } \\
\hline & \multicolumn{3}{|c|}{ LMS-parameters } & \multicolumn{3}{|c|}{ Percentiles $\left(\mathrm{ml} / \mathrm{m}^{2}\right)$} & \multicolumn{3}{|c|}{ LMS-parameters } & \multicolumn{3}{|c|}{ Percentiles $\left(\mathrm{ml} / \mathrm{m}^{2}\right)$} \\
\hline & L & $M$ & $S$ & P3 & P50 & P97 & L & $M$ & $S$ & P3 & P50 & P97 \\
\hline 6 & 1.378 & 36.715 & 0.263 & 14 & 37 & 55 & 1.806 & 33.342 & 0.191 & 20 & 39 & 68 \\
\hline 7 & 1.378 & 38.610 & 0.246 & 17 & 39 & 56 & 1.806 & 48.385 & 0.203 & 22 & 43 & 71 \\
\hline 8 & 1.378 & 40.291 & 0.229 & 20 & 40 & 57 & 1.806 & 51.247 & 0.205 & 24 & 47 & 73 \\
\hline 9 & 1.378 & 41.762 & 0.212 & 22 & 42 & 58 & 1.806 & 51.742 & 0.205 & 26 & 49 & 74 \\
\hline 10 & 1.378 & 43.375 & 0.197 & 25 & 43 & 59 & 1.806 & 52.579 & 0.204 & 28 & 52 & 75 \\
\hline 11 & 1.378 & 45.120 & 0.183 & 27 & 45 & 61 & 1.806 & 54.891 & 0.200 & 30 & 54 & 76 \\
\hline 12 & 1.378 & 46.671 & 0.171 & 29 & 47 & 62 & 1.806 & 56.348 & 0.197 & 32 & 57 & 77 \\
\hline 13 & 1.378 & 47.784 & 0.161 & 31 & 48 & 62 & 1.806 & 57.830 & 0.193 & 33 & 59 & 78 \\
\hline 14 & 1.378 & 48.331 & 0.152 & 33 & 48 & 62 & 1.806 & 59.473 & 0.188 & 34 & 61 & 79 \\
\hline 15 & 1.378 & 48.581 & 0.142 & 34 & 49 & 62 & 1.806 & 61.042 & 0.181 & 35 & 63 & 80 \\
\hline 16 & 1.378 & 49.112 & 0.131 & 36 & 49 & 61 & 1.806 & 63.114 & 0.171 & 37 & 65 & 81 \\
\hline 17 & 1.378 & 50.353 & 0.120 & 38 & 50 & 62 & 1.806 & 64.322 & 0.161 & 38 & 67 & 82 \\
\hline 18 & 1.378 & 52.583 & 0.111 & 40 & 53 & 64 & 1.806 & 66.227 & 0.145 & 40 & 69 & 84 \\
\hline 19 & 1.378 & 55.860 & 0.103 & 44 & 56 & 67 & 1.806 & 72.157 & 0.110 & 43 & 71 & 85 \\
\hline 20 & 1.378 & 59.928 & 0.097 & 48 & 60 & 71 & 1.806 & 77.498 & 0.064 & 45 & 72 & 86 \\
\hline
\end{tabular}

LMS: $L=L a m b d a$ (skewness of the distribution), $M=M u$ (median), $S=$ Sigma (variance)

Standard deviation score $(S D S)=[(X / M) L-1] /\left(L^{*} S\right)$, where $X$ is the measured atrial volume in $\mathrm{ml} / \mathrm{m} 2$ and $L, M$ and $S$ are the values interpolated for the child's age; lower and upper limits correspond to a score of -2 and 2 and to the $3 \mathrm{rd}$ and 97 th percentile, respectively

${ }^{\text {a }}$ Age in years

Table 21 Normal left atrial and right atrial volume in girls; LMS parameters to calculate z-scores and percentiles relative to age according to reference [47]

\begin{tabular}{|c|c|c|c|c|c|c|c|c|c|c|c|c|}
\hline \multirow[t]{3}{*}{$\mathrm{Age}^{\mathrm{a}}$} & \multicolumn{6}{|c|}{ Left atrium } & \multicolumn{6}{|c|}{ Right atrium } \\
\hline & \multicolumn{3}{|c|}{ LMS-parameters } & \multicolumn{3}{|c|}{ Percentiles $\left(\mathrm{ml} / \mathrm{m}^{2}\right)$} & \multicolumn{3}{|c|}{ LMS-parameters } & \multicolumn{3}{|c|}{ Percentiles $\left(\mathrm{ml} / \mathrm{m}^{2}\right)$} \\
\hline & $\mathbf{L}$ & M & $S$ & P3 & P50 & P97 & L & $M$ & $S$ & P3 & P50 & P97 \\
\hline 4 & -1.100 & 37.566 & 0.248 & 22 & 34 & 44 & 0.889 & 47.196 & 0.328 & 18 & 47 & 79 \\
\hline 5 & -0.956 & 38.333 & 0.242 & 23 & 36 & 46 & 0.774 & 47.386 & 0.318 & 20 & 47 & 80 \\
\hline 6 & -0.717 & 39.568 & 0.234 & 25 & 39 & 50 & 0.587 & 47.733 & 0.302 & 23 & 48 & 80 \\
\hline 7 & -0.478 & 40.739 & 0.225 & 26 & 41 & 53 & 0.421 & 48.181 & 0.284 & 25 & 48 & 80 \\
\hline 8 & -0.239 & 41.934 & 0.217 & 28 & 43 & 55 & 0.266 & 48.837 & 0.265 & 28 & 49 & 80 \\
\hline 9 & 0.000 & 43.072 & 0.208 & 28 & 44 & 56 & 0.106 & 49.868 & 0.244 & 30 & 50 & 80 \\
\hline 10 & 0.239 & 43.953 & 0.199 & 28 & 44 & 56 & -0.033 & 51.098 & 0.221 & 33 & 51 & 80 \\
\hline 11 & 0.478 & 44.548 & 0.191 & 29 & 44 & 57 & -0.071 & 52.283 & 0.197 & 35 & 52 & 78 \\
\hline 12 & 0.717 & 45.080 & 0.182 & 29 & 45 & 58 & 0.029 & 53.388 & 0.175 & 38 & 53 & 76 \\
\hline 13 & 0.956 & 45.636 & 0.173 & 30 & 45 & 59 & 0.262 & 54.329 & 0.157 & 39 & 54 & 73 \\
\hline 14 & 1.195 & 46.118 & 0.165 & 30 & 46 & 60 & 0.595 & 55.205 & 0.147 & 40 & 55 & 72 \\
\hline 15 & 1.434 & 46.070 & 0.156 & 30 & 47 & 60 & 0.991 & 55.815 & 0.145 & 40 & 56 & 72 \\
\hline 16 & 1.673 & 45.343 & 0.148 & 30 & 46 & 59 & 1.419 & 56.153 & 0.148 & 38 & 56 & 72 \\
\hline 17 & 1.912 & 44.258 & 0.139 & 29 & 44 & 57 & 1.852 & 56.470 & 0.155 & 36 & 56 & 72 \\
\hline 18 & 2.151 & 43.116 & 0.130 & 28 & 42 & 55 & 2.276 & 57.000 & 0.164 & 31 & 57 & 73 \\
\hline
\end{tabular}

LMS, $\mathrm{L}=$ Lambda (skewness of the distribution), $M=M u$ (median), $S=$ Sigma (variance)

Standard deviation score $(S D S)=\left[(X / M)^{L}-1\right] /\left(L^{*} S\right)$, where $X$ is the measured atrial volume in $\mathrm{ml} / \mathrm{m}^{2}$ and $\mathrm{L}, \mathrm{M}$ and $\mathrm{S}$ are the values interpolated for the child's age; lower and upper limits correspond to a score of -2 and 2 and to the $3 \mathrm{rd}$ and 97 th percentile, respectively

${ }^{\text {a }}$ Age in years 
Table 22 Reference, cardiac chamber size in the athlete

\begin{tabular}{|c|c|c|c|}
\hline First author, year & CMR technique & n, gender, sports intensity & $\begin{array}{l}\text { Age range } \\
\text { (years) }\end{array}$ \\
\hline \multirow[t]{6}{*}{ Prakken, 2010 [50] } & 1.5 T, short axis bSSFP, papillary muscles included in LV volume & 83 , male, regular athletes (9-18 h/week) & $18-39$ \\
\hline & & 46 , male, elite athletes (> 18 h/week) & $18-39$ \\
\hline & & 60 , female, regular athletes (9-18 h/week) & $18-39$ \\
\hline & & 33 , female, elite athletes (> $18 \mathrm{~h} /$ week) & $18-39$ \\
\hline & & 56 , male, non-athletes & $18-39$ \\
\hline & & 58 , female, non-athletes & $18-39$ \\
\hline
\end{tabular}

$n$ number of study subjects, bSSFP balanced steady-state free precession

Table 23 Left ventricular parameters for adult athletes (papillary muscles included in LV volume) according to reference [50]

\begin{tabular}{|c|c|c|c|c|c|c|}
\hline \multirow[t]{2}{*}{ Parameter } & \multicolumn{2}{|c|}{ Non-athletes [mean \pm SD (LL-UL) $\left.{ }^{c}\right]$} & \multicolumn{2}{|c|}{$\begin{array}{l}\text { Regular athletes }{ }^{\mathrm{a}} \text { [mean } \pm \text { SD (LL- } \\
\left.\text { UL) }^{\mathrm{c}}\right]\end{array}$} & \multicolumn{2}{|c|}{ Elite athletes ${ }^{b}\left[\right.$ mean $\pm S D\left(\right.$ LL-UL) $\left.{ }^{c}\right]$} \\
\hline & Men $(n=56)$ & Women $(n=58)$ & Men $(n=83)$ & Women $(n=60)$ & Men $(n=46)$ & Women $(n=33)$ \\
\hline LVEDV (ml) & $201 \pm 33(135-267)$ & $156 \pm 22(112-200)$ & $250 \pm 32(186-314)$ & $194 \pm 27(140-248)$ & $261 \pm 39(183-339)$ & $199 \pm 31(137-261)$ \\
\hline LVEDV/BSA $\left(\mathrm{ml} / \mathrm{m}^{2}\right)$ & $101 \pm 15(71-131)$ & $90 \pm 11(68-112)$ & $123 \pm 13(97-149)$ & $107 \pm 14(79-135)$ & $129 \pm 17(95-163)$ & $107 \pm 14(79-135)$ \\
\hline LVESV (ml) & $87 \pm 19(49-125)$ & $65 \pm 13(39-91)$ & $108 \pm 20(68-148)$ & $86 \pm 15(56-116)$ & $117 \pm 24(69-165)$ & $85 \pm 20(45-125)$ \\
\hline LVESV/BSA $\left(\mathrm{ml} / \mathrm{m}^{2}\right)$ & $43 \pm 10(23-63)$ & $37 \pm 7(23-51)$ & $53 \pm 9(35-71)$ & $48 \pm 8(32-64)$ & $58 \pm 11(36-80)$ & $46 \pm 11(24-68)$ \\
\hline LVM (g) & $95 \pm 20(55-135)$ & $60 \pm 11(38-82)$ & $125 \pm 22(81-169)$ & $84 \pm 17(50-118)$ & $139 \pm 28(83-195)$ & $92 \pm 15(62-122)$ \\
\hline LVM/BSA (g/m²) & $48 \pm 9(30-66)$ & $34 \pm 6(22-46)$ & $62 \pm 11(40-84)$ & $46 \pm 9(28-64)$ & $69 \pm 13(43-95)$ & $50 \pm 8(34-66)$ \\
\hline LVEF (\%) & $57 \pm 6(45-69)$ & $58 \pm 5(48-68)$ & $57 \pm 5(47-67)$ & $55 \pm 4(47-63)$ & $55 \pm 5(45-65)$ & $58 \pm 7(44-72)$ \\
\hline $\max$. IVS (mm) & $10 \pm 1(8-12)$ & $5 \pm 1(3-7)$ & $11 \pm 1(9-13)$ & $9 \pm 1(7-11)$ & $11 \pm 1(9-13)$ & $9 \pm 1(7-11)$ \\
\hline
\end{tabular}

$S D$ standard deviation, $L L$ lower limit, UL upper limit, $n$ number of study subjects, $L V$ left ventricular, $E D V$ end-diastolic volume, ESV end-systolic volume, EF ejection fraction, LVM left ventricular mass, max. IVS maximal thickness of the interventricular septum, BSA body surface area

a $9-18 \mathrm{~h}$ sports activity/week

${ }^{\mathrm{b}}>18 \mathrm{~h}$ sports activity/week

${ }^{\mathrm{C}}$ Calculated as mean $\pm 2 * \mathrm{SD}$

Table 24 Right ventricular parameters for adult athletes (papillary muscles included in right ventricular volume) according to reference [50]

\begin{tabular}{|c|c|c|c|c|c|c|}
\hline \multirow[t]{2}{*}{ Parameter } & \multicolumn{2}{|c|}{ Non-athletes [mean \pm SD (LL-UL) $\left.{ }^{c}\right]$} & \multicolumn{2}{|c|}{ 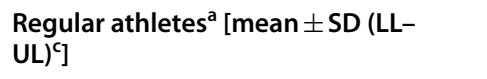 } & \multicolumn{2}{|c|}{ Elite athletes ${ }^{b}\left[\right.$ mean $\pm S D\left(\right.$ LL-UL) $\left.{ }^{c}\right]$} \\
\hline & Men $(n=56)$ & & Men $(n=83)$ & Women $(n=60)$ & Men $(n=46)$ & Women $(n=33)$ \\
\hline RVEDV (ml) & $223 \pm 40(143-303)$ & $166 \pm 23(120-212)$ & $277 \pm 36(205-349)$ & $209 \pm 29(151-267)$ & $291 \pm 48(195-387)$ & $219 \pm 35(149-289)$ \\
\hline RVEDV/BSA $\left(\mathrm{ml} / \mathrm{m}^{2}\right)$ & $111 \pm 18(75-147)$ & $96 \pm 12(72-120)$ & $136 \pm 16(104-168)$ & $115 \pm 15(85-145)$ & $144 \pm 20(104-184)$ & $118 \pm 17(84-152)$ \\
\hline RVESV (ml) & $108 \pm 24(60-156)$ & $75 \pm 13(49-101)$ & $135 \pm 25(85-185)$ & $102 \pm 17(68-136)$ & $148 \pm 30(88-208)$ & $103 \pm 24(55-151)$ \\
\hline RVESV/BSA $\left(\mathrm{ml} / \mathrm{m}^{2}\right)$ & $54 \pm 12(30-78)$ & $43 \pm 7(29-57)$ & $66 \pm 12(42-90)$ & $57 \pm 9(39-75)$ & $73 \pm 13(47-99)$ & $56 \pm 13(30-82)$ \\
\hline RVM (g) & $23 \pm 5(13-33)$ & $18 \pm 4(10-26)$ & $29 \pm 6(17-41)$ & $23 \pm 4(15-31)$ & $30 \pm 6(18-42)$ & $25 \pm 5(15-35)$ \\
\hline RVM/BSA $\left(\mathrm{g} / \mathrm{m}^{2}\right)$ & $12 \pm 2(8-16)$ & $10 \pm 2(6-14)$ & $14 \pm 3(8-20)$ & $13 \pm 2(9-17)$ & $15 \pm 2(11-19)$ & $14 \pm 3(8-20)$ \\
\hline RVEF (\%) & $52 \pm 5(42-62)$ & $55 \pm 5(45-65)$ & $51 \pm 4(43-59)$ & $51 \pm 4(43-59)$ & $50 \pm 4(42-58)$ & $53 \pm 7(39-67)$ \\
\hline
\end{tabular}

$S D$ standard deviation, $L L$ lower limit, UL upper limit, $n$ number of study subjects, $R V$ right ventricular, EDV end-diastolic volume, ESV end-systolic volume, $E F$ ejection fraction, RVM right ventricular mass, $B S A$ body surface area

a $9-18 \mathrm{~h}$ sports activity/week

b $>18 \mathrm{~h}$ sports activity/week

${ }^{\mathrm{c}}$ Calculated as mean $\pm 2 * \mathrm{SD}$ 
Table 25 References, normal thickness of the compact left ventricular myocardium in the adult

\begin{tabular}{llll}
\hline First author, year & CMR technique & n, male:female & Age range (years) \\
\hline Dawson, 2011 [56] & 1.5 T, short axis bSSFP, 16 segments (apex excluded) & $60: 60$ & $20-80$ \\
Kawel, 2012 [55] & 1.5 T, short (16 segments, apex excluded) and long axis (12 segments) bSSFP & $131: 169$ & $54-91$ \\
Le Ven, 2015 [14] & 1.5 T, short axis bSSFP; 16 segments (apex excluded) & $196: 238$ & $18-36$ \\
Yeon, 2015 [25] & 1.5 T, short axis bSSFP; 2 segments (basal inferolateral and anteroseptal) & $340: 512$ & (men: 61 12 ; women: 62 \pm 9 ) $^{\text {a }}$ \\
Aquaro, 2017 [18] & 1.5 T, short axis bSSFP; 2 segments (basal anterior septum, basal inferolateral & $173: 135$ & $15-80$ \\
& wall) & & \\
\hline
\end{tabular}

$n$ number of study subjects, bSSFP balanced steady-state free precession

${ }^{a}$ Age range not provided in original publication

ranges. For the purposes of this review, that meta-analysis included multiple publications with overlapping/ redundant study populations, small sample size $(<40$ subjects in most studies) and did not take into account marked differences in analysis methods noted above. While useful to display overall trends in the literature for the athletes heart, the aforementioned meta-analysis was therefore not included in this study.

\section{Normal thickness of the compact left ventricular myocardium in adults CMR acquisition parameters}

Normal values of the thickness of the compact LV myocardium have been shown to vary by type of pulse sequence (FGRE versus bSSFP) [53, 54]. For the purposes of this review, only bSSFP normal values are shown.

\section{CMR analysis methods}

In this review LV myocardial thickness refers to measurements of the thickness of the compact LV myocardium obtained at end-diastole (Fig. 7). Papillary muscles and trabeculations are excluded from measurement of the thickness of the compact LV myocardium.

Measures of LV myocardial thickness vary by the plane of acquisition (SAx versus long axis) [55]. Measurements obtained on long axis images at the basal and mid-cavity level have been shown to be significantly greater compared to measurements on corresponding SAx images, whereas measurements obtained at the apical level of long axis images are significantly lower compared to SAx images.

\section{Demographic parameters}

LV myocardial thickness is greater in men than women $[14,18,25,55,56]$. There are also small differences in LV myocardial thickness in relationship to ethnicity and body size, but these variations are not likely to have clinical significance [55]. Regarding age, one study of 120 healthy subjects age 20-80 years reported an increase in myocardial thickness with age-starting after the fourth decade [56]. In the study by Kawel el al. of 300 normal individuals without hypertension, smoking history or diabetes, there was no statistically significant difference in LV myocardial thickness with age [55].

\section{Studies included in this review}

There are five publications of a systematic analysis of LV myocardial thickness based on bSSFP imaging at $1.5 \mathrm{~T}$ with a sample size $>40$ healthy subjects per gender and a detailed description of the measurement technique (Table 25). Dawson et al. and Le Ven et al. published measurements for all 16 segments (apex excluded) obtained on short axis images (Table 26) [14, 56]. Kawel et al. published normal values of LV myocardial thickness for long and SAx imaging for 12 and 16 segments, respectively (Tables 26, 27) [55]. Yeon et al. and Aquaro et al. obtained measurements for only two myocardial segments on SAx images (Table 26) [18, 25].

\section{Normal values of left ventricular trabeculation CMR acquisition parameters}

CMR methods used to assess LV trabeculation (Table 28) are based on the bSSFP technique to leverage on the blood-myocardial contrast it provides. The key methods are illustrated in Fig. 7.

\section{CMR analysis methods}

No uniformly accepted convention has been used for analyzing trabeculation. At least seven different measurement approaches have been described (Table 28). Principally these methods measure trabeculation in the LV either in terms of the trabeculated layer's thickness, mass, volume, or fractal complexity, with or without adjusting for the thickness, mass or volume of the adjacent compacted myocardium. Tables of normal values for trabeculation should specify the phase of the cardiac cycle in which measurements were taken together with imaging planes used. When reporting trabeculation mass, volume or fractal complexity, tables should specify whether papillary muscles were included or excluded in the trabecular assessment. Where 
semi-automated segmentation of trabecular contours is undertaken, the type of algorithm used may impact subsequent results so the methods must specify the algorithm in detail [57].

Table 29 provides normal adult values for thickness of the trabeculated LV myocardium, on a segment-by-segment basis. Table 30 provides normal values for mass and volume of trabeculation. Trabeculation mass ratio has additionally been reported $[12,58,59]$ but measurement heterogeneity across studies, with respect to handling of the blood pool between trabeculations and inclusion/exclusion of papillary muscles, has led to differing definitions and no consensus normal values.

Tables 31 and 32 provide normal values for LV trabeculation measured as a fractal dimension. Four fractal parameters for quantifying LV trabeculation [59] include global LV, maximal basal, maximal mid and maximal apical fractal dimension. To derive the global LV fractal dimension, the fractal dimensions from each slice in the LV stack (Fig. 7d) were averaged; to derive local fractal characteristics, the maximal fractal dimension in the basal, mid and apical thirds of the left ventricle were recorded [59].

Normal values by this approach for global LV and maximal apical fractal dimension are presented in Table 31. Methodological developments for fractal analysis of the left ventricle are ongoing [60].

\section{Demographic parameters}

In the largest published reference cohort $(n=323)$ [61], there was no relationship between maximal non-compacted (NC)/compacted (C) wall thickness ratio and age, gender, race/ethnicity, height or weight.

For segment-by-segment (whole-heart) $\mathrm{NC} / \mathrm{C}$ ratio [56], there was also no significant difference between genders, but age-related differences were present: the thickness of the trabeculated myocardium generally increased until the 3rd decade and subsequently decreased. This trend was significant in the anterior $(1,7,13)$ and apical inferior segments, but not in the remainder of segments [56].
Using the fractal dimension, ethnicity was shown to influence LV trabeculation parameters, with greater endocardial complexity (i.e. higher fractal dimension) demonstrated in healthy blacks as opposed to healthy whites, and greater complexity demonstrated in Whites, African American and Hispanics compared to Chinese Americans [62].

\section{Studies included in this review}

For the purpose of this review, only cohorts of 40 or more normal subjects using bSSFP CMR technique have been included. Data from population-based studies where exclusions for comorbidities was undertaken have also been included $[61,62]$. The majority of reported normal values were derived at $1.5 \mathrm{~T}$ although a few $3 \mathrm{~T}$ studies have also been undertaken (see Table 28). Inclusion criteria for reported tables included a full description of the subject cohort (including the analysis methods used), age and gender of subjects. One study evaluated elite male athletes which was not deemed to be representative of the average population and was therefore not included in this review [63].

The caliper-based linear measurement of thickness of trabeculation [61] has progressively evolved into more complex metrics: the maximal $\mathrm{NC} / \mathrm{C}$ thickness ratio has been measured by at least four groups $[58,59,61,64]$ but reported normal values were too discordant for calculation of weighted means in this review (thus not shown in Table 29). The inter-study discordance of maximal NC/C parameters may stem from the subjective selection by readers of the visually most trabeculated segment/s for analysis (Fig. 7b). The largest of these studies, which also included reproducibility assessment, reported median values for normal adult maximal $\mathrm{NC} / \mathrm{C}$ thickness of 2.2 [5th and 95th percentile: 1.0, 4.6] [61]. Other studies opted for a more systematic segment-by-segment analysis of thickness of trabeculation but still methodologies differed: Dawson et al. [56]. measured the maximal thickness of trabeculated myocardium per segment (Fig. 7a), whereas Tizon [65] measured the average of 20-30

\footnotetext{
(See figure on next page.)

Fig. 7 Example of measurement approaches for LV trabeculation. a End-diastolic thickness (in mm) of trabeculation according to the methodology in [56]: 3 slices representing base, mid and apex were selected from within the entire LV stack; trabeculated myocardial thickness was measured per slice; segment 17 excluded from analysis; authors do not clarify whether papillary muscles had been included or excluded from the trabecular measurement-in this reproduction we have excluded papillary muscles. b Maximal non-compacted (NC, red lines)/compacted (c, orange lines) wall thickness ratio according to the methodology in [61]: papillary muscles that were clearly observed as compact tubular structures were not included in the measurements; measurements in $\mathrm{mm}$ are shown in white and the maximal NC/C parameter highlighted in blue. c Trabeculation mass according to the methodology in [12]: the endocardial contour (red) was manually drawn; the trabecular contour (orange) was automatically segmented and papillary muscles (blue) that were included in the compact myocardial mass, were semi-automatically segmented; all slices of the LV short axis stack were analyzed. d Fractal dimension according to the methodology in [60]: using a semi-automatic level-set segmentation with bias field correction; all slices of the LV short axis stack are analyzed except for the apical slice; fractal dimensions per slice reported in the top right corner
} 
a
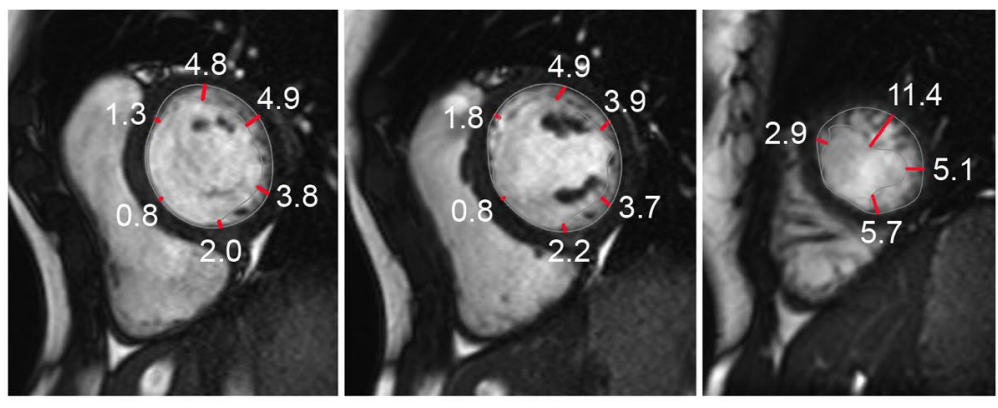

b

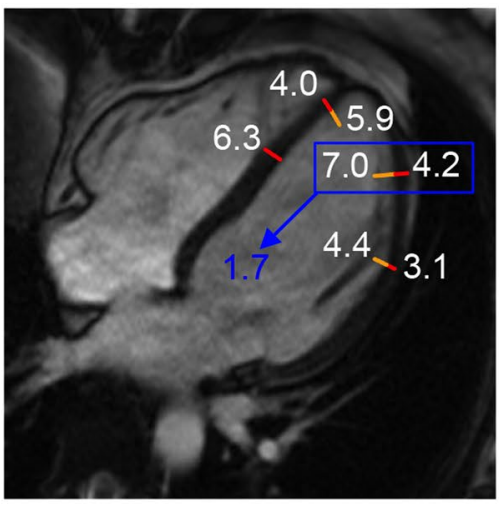

c
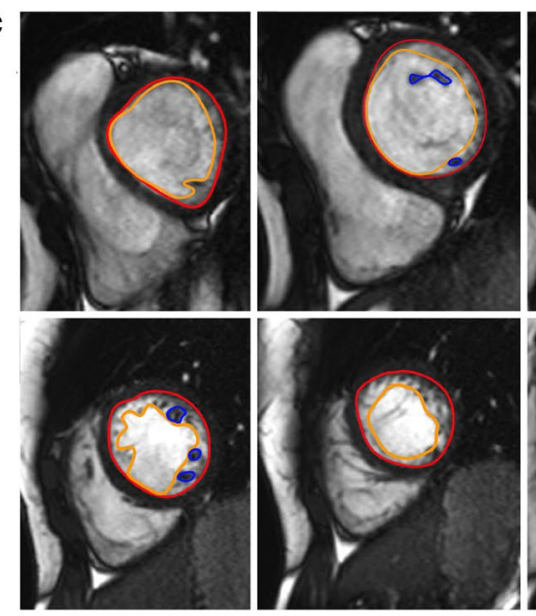

d
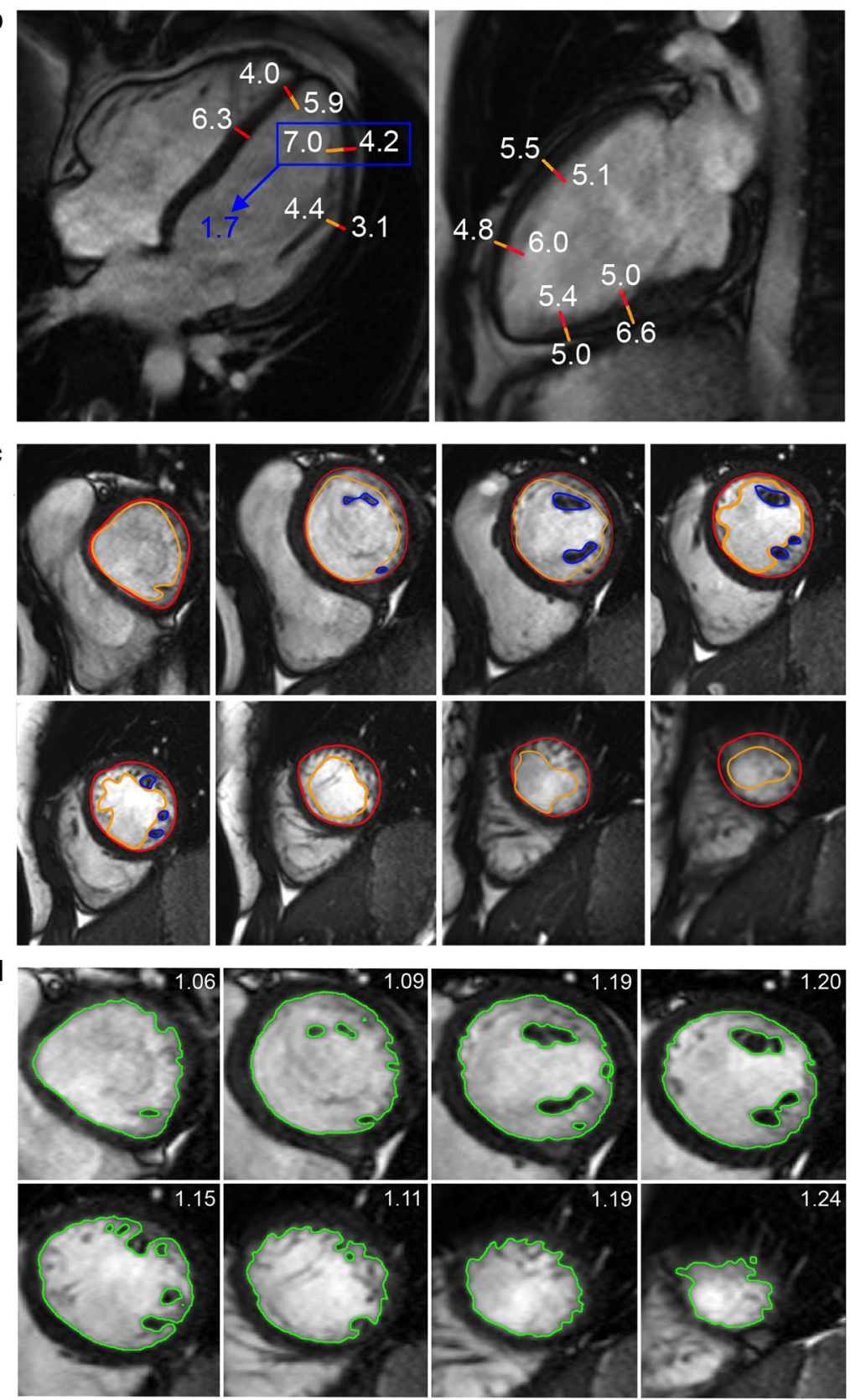

Fig. 7 (See legend on previous page.) 
Table 26 Normal left ventricular myocardial thickness (in $\mathrm{mm}$ ) in the adult measured on short axis images for men and women

\begin{tabular}{|c|c|c|c|c|c|c|c|c|c|}
\hline \multirow[t]{2}{*}{ Level } & \multirow[t]{2}{*}{ Segment } & \multicolumn{4}{|c|}{ Men } & \multicolumn{4}{|c|}{ Women } \\
\hline & & $n$ & $\operatorname{Mean}_{p}$ & $S D_{p}$ & $\overline{L L-U L^{c}}$ & $n$ & $\operatorname{Mean}_{\mathrm{p}}$ & $S D_{p}$ & LL-ULc \\
\hline \multirow[t]{6}{*}{ Basal } & $1^{\mathrm{a}}$ & 387 & 7.8 & 1.3 & $5-10$ & 467 & 6.4 & 1.1 & $4-9$ \\
\hline & $2^{b}$ & 900 & 9.0 & 1.4 & $6-12$ & 1114 & 7.6 & 1.2 & $5-10$ \\
\hline & $3^{a}$ & 387 & 8.8 & 1.2 & $6-11$ & 467 & 7.3 & 1.0 & $5-9$ \\
\hline & $4^{\mathrm{a}}$ & 387 & 7.9 & 1.2 & $6-10$ & 467 & 6.4 & 1.0 & $4-8$ \\
\hline & $5^{b}$ & 900 & 7.7 & 1.2 & $5-10$ & 1114 & 6.3 & 1.1 & $4-9$ \\
\hline & $6^{a}$ & 387 & 7.5 & 1.2 & $5-10$ & 467 & 6.1 & 1.0 & $4-8$ \\
\hline \multirow[t]{6}{*}{ Mid-cavity } & $7^{\mathrm{a}}$ & 387 & 6.7 & 1.2 & 4-9 & 467 & 5.6 & 1.0 & $4-8$ \\
\hline & $8^{\mathrm{a}}$ & 387 & 7.4 & 1.3 & $5-10$ & 467 & 6.1 & 1.0 & $4-8$ \\
\hline & $9^{a}$ & 387 & 7.9 & 1.2 & $6-10$ & 467 & 6.6 & 1.0 & $5-9$ \\
\hline & $10^{\mathrm{a}}$ & 387 & 7.0 & 1.2 & $5-9$ & 467 & 5.8 & 1.0 & $4-8$ \\
\hline & $11^{\mathrm{a}}$ & 387 & 6.5 & 1.4 & 4-9 & 467 & 5.3 & 1.0 & $3-7$ \\
\hline & $12^{\mathrm{a}}$ & 387 & 6.6 & 1.2 & $4-9$ & 467 & 5.5 & 1.1 & $4-8$ \\
\hline \multirow[t]{4}{*}{ Apical } & $13^{\mathrm{a}}$ & 387 & 6.5 & 1.2 & 4-9 & 467 & 5.9 & 1.3 & $3-9$ \\
\hline & $14^{\mathrm{a}}$ & 387 & 6.8 & 1.3 & 4-9 & 467 & 5.8 & 1.1 & $4-8$ \\
\hline & $15^{\mathrm{a}}$ & 387 & 6.1 & 1.1 & $4-8$ & 467 & 5.2 & 1.0 & $3-7$ \\
\hline & $16^{\mathrm{a}}$ & 387 & 6.2 & 1.1 & $4-8$ & 467 & 5.6 & 1.0 & $4-8$ \\
\hline
\end{tabular}

Segments: 1: basal anterior, 2: basal anteroseptal, 3: basal inferoseptal, 4: basal inferior, 5: basal inferolateral, 6: basal anterolateral, 7: mid anterior, 8: mid anteroseptal, 9: mid inferoseptal, 10: mid inferior, 11: mid inferolateral, 12: mid anterolateral, 13: apical anterior, 14: apical septal, 15: apical inferior, 16: apical lateral

$n$ number of study subjects included in the weighted mean values, mean ${ }_{p}$ pooled weighted mean, $S D_{p}$ pooled standard deviation, $L L$ lower limits, $U L$ upper limits

a Pooled weighted values from references $[14,55,56]$

${ }^{\mathrm{b}}$ Pooled weighted values from references $[14,18,25,55,56]$

${ }^{c}$ Calculated as mean p $\pm 2 \mathrm{SD}_{\mathrm{p}}$

Table 27 Normal left ventricular myocardial thickness (in $\mathrm{mm}$ ) in the adult measured on long axis images for men and women according to reference [55]

\begin{tabular}{|c|c|c|c|c|c|c|c|}
\hline \multirow[t]{2}{*}{ Level } & \multirow[t]{2}{*}{ Region } & \multicolumn{3}{|c|}{$\operatorname{Men}(n=131)$} & \multicolumn{3}{|c|}{ Women $(n=169)$} \\
\hline & & Mean & SD & LL-UL ${ }^{a}$ & Mean & SD & LL-UL ${ }^{a}$ \\
\hline \multirow[t]{5}{*}{ Basal } & Anterior & 8.2 & 1.3 & $6-11$ & 7 & 1.1 & $5-9$ \\
\hline & Inferior & 8.2 & 1.3 & $6-10$ & 6.7 & 1.1 & $5-9$ \\
\hline & Septal & 9.1 & 1.3 & $7-12$ & 7.3 & 1.1 & $5-10$ \\
\hline & Lateral & 7.6 & 1.3 & $5-10$ & 6 & 1.1 & $4-8$ \\
\hline & Mean & 8.3 & 1.0 & $6-10$ & 6.8 & 0.9 & $5-9$ \\
\hline \multirow[t]{5}{*}{ Mid-cavity } & Anterior & 6 & 1.3 & $3-9$ & 4.9 & 1.1 & $3-7$ \\
\hline & Inferior & 7.7 & 1.3 & $5-10$ & 6.5 & 1.1 & $4-9$ \\
\hline & Septal & 8.3 & 1.3 & $6-11$ & 6.8 & 1.1 & $5-9$ \\
\hline & Lateral & 6.6 & 1.3 & $4-9$ & 5.3 & 1.1 & $3-8$ \\
\hline & Mean & 7.2 & 1.0 & $5-9$ & 6 & 1 & $4-8$ \\
\hline \multirow[t]{5}{*}{ Apical } & Anterior & 5.1 & 1.3 & $3-8$ & 4.2 & 1.1 & $2-6$ \\
\hline & Inferior & 5.8 & 1.3 & $3-8$ & 5 & 1.1 & $3-7$ \\
\hline & Septal & 5.8 & 1.3 & $3-8$ & 5 & 1.1 & $3-7$ \\
\hline & Lateral & 5.5 & 1.3 & $3-8$ & 4.6 & 1.1 & $2-7$ \\
\hline & Mean & 5.6 & 1.0 & $4-8$ & 4.7 & 0.9 & $3-7$ \\
\hline
\end{tabular}

$n$ number of study subjects, SD standard deviation, LL lower limits, UL upper limits

a Calculated as mean $\pm 2 * \mathrm{SD}$ 
Table 28 References, normal thickness, mass, ratios and fractal dimension of the left ventricular trabeculated (non-compacted) myocardium in the adult

\begin{tabular}{|c|c|c|c|}
\hline First author, year & CMR technique & n, male:female & Age range (years) \\
\hline \multicolumn{4}{|c|}{ Trabeculation thickness (thickness of the trabeculated [non-compacted] LV myocardium) } \\
\hline Dawson, 2011 [56] & 1.5T, short axis bSSFP, maximal thickness per segment at diastole and systole & $60: 60$ & $20-80$ \\
\hline \multicolumn{4}{|c|}{$\mathrm{NC} / \mathrm{C}$ thickness ratio (thickness of trabeculated [non-compacted] LV myocardium/ thickness of compact LV myocardium) } \\
\hline Dawson, 2011 [56] & $\begin{array}{l}1.5 \mathrm{~T} \text {, short axis bSSFP, NC/C thickness ratio per segment measured manually } \\
\text { at the "peak of the most prominent trabeculae in each segment" at diastole } \\
\text { and systole }\end{array}$ & $60: 60$ & $20-80$ \\
\hline Kawel, 2012 [61] & $\begin{array}{l}1.5 \mathrm{~T} \text {, long axis bSSFP at diastole, maximal NC/C thickness ratio of } 12 \mathrm{seg} \text { - } \\
\text { ments }\end{array}$ & 192:175 & 54-91 \\
\hline Captur, 2013 [59] & $\begin{array}{l}1.5 \mathrm{~T} \text {, long axis bSSFP at diastole, maximal NC/C thickness ratio of } 16 \text { seg- } \\
\text { ments }\end{array}$ & 40 (total)* $^{*}$ & 18-85 \\
\hline Tizón-Marcos, 2014 [65] & $\begin{array}{l}\text { 1.5 T, long- and short axis bSSFP, mean NC/C thickness ratio per segment } \\
\text { measured semi-automatically by the centerline method (average of } 20-30 \\
\text { chords/segment) at diastole and systole }\end{array}$ & 45:55 & $18-35$ \\
\hline Amzulescu, 2015 [58] & $\begin{array}{l}1.5 \mathrm{~T} \text { and } 3 \mathrm{~T} \text {, long axis bSSFP at diastole, maximal NC/C thickness ratio of } 16 \\
\text { segments }\end{array}$ & $22: 26$ & $(60 \pm 10)^{* *}$ \\
\hline André, 2015 [64] & 1.5T, long axis bSSFP at diastole,maximal NC/C thickness ratio of 16 segments & $58: 59$ & $20->50$ \\
\hline \multicolumn{4}{|c|}{ Trabeculation mass (mass of the trabeculated [non-compacted] LV myocardium) } \\
\hline Bentatou, 2018 [12] & $\begin{array}{l}1.5 \mathrm{~T} \text {, short axis bSSFP at diastole, papillary muscles and blood between } \\
\text { trabeculae excluded }\end{array}$ & $70: 70$ & $20-69$ \\
\hline \multicolumn{4}{|c|}{ Trabeculation volume (volume of the trabeculated [non-compacted] LV myocardium) } \\
\hline André, 2015 [64] & $\begin{array}{l}1.5 \mathrm{~T} \text {, short axis bSSFP, blood between trabeculae included, papillary muscles } \\
\text { excluded }\end{array}$ & $58: 59$ & $20->50$ \\
\hline \multicolumn{4}{|c|}{$\mathrm{NC} / \mathrm{C}$ mass ratio (mass of trabeculated [non-compacted] LV myocardium/ mass of compact LV myocardium) } \\
\hline Amzulescu, 2015 [58] & $\begin{array}{l}1.5 \mathrm{~T} \text { and } 3 \mathrm{~T} \text {, short axis bSSFP at diastole, mass of trabeculated myocardium } \\
\text { includes trabeculae and blood between trabeculae, papillary muscles } \\
\text { excluded from trabeculated and compact mass }\end{array}$ & 22:26 & $(60 \pm 10)^{b}$ \\
\hline Bentatou, 2018 [12] & $\begin{array}{l}1.5 \mathrm{~T} \text {, short axis bSSFP at diastole, blood between trabeculae excluded from } \\
\text { mass of trabeculated myocardium, papillary muscles included in mass of } \\
\text { compact myocardium }\end{array}$ & $70: 70$ & $20-69$ \\
\hline \multicolumn{4}{|c|}{ NC/TM (mass of trabeculated [non-compacted] LV myocardium/ total LV myocardial mass [trabeculated + compact LV myocardial mass]) } \\
\hline Captur, 2013 [59] & $\begin{array}{l}1.5 \mathrm{~T} \text {, short axis bSSFP at diastole, mass of trabeculated myocardium includes } \\
\text { trabeculae and blood between trabeculae, papillary muscles included in } \\
\text { mass of compact myocardium }\end{array}$ & $40(\text { total) })^{\mathrm{a}}$ & 18-85 \\
\hline \multicolumn{4}{|c|}{ Fractal dimension (fractal complexity of LV trabeculated [non-compacted] myocardium) } \\
\hline Captur, 2013 [59] & $\begin{array}{l}1.5 \mathrm{~T} \text {, short axis bSSFP at diastole, papillary muscles included in the endocar- } \\
\text { dial complexity }\end{array}$ & 51:54 (75 white, 30 black) & 18-85 \\
\hline Captur 2015 [62] & $\begin{array}{l}1.5 \mathrm{~T} \text {, short axis bSSFP at diastole, papillary muscles included in the endocar- } \\
\text { dial complexity }\end{array}$ & 279:325 & 46-91 \\
\hline Cai, 2017 [66] & $\begin{array}{l}\text { 3T, short axis bSSFP at diastole, papillary muscles included in the endocardial } \\
\text { complexity }\end{array}$ & $91: 89$ & $20-69$ \\
\hline
\end{tabular}

$n$ number of study subjects, LV left ventricular, bSSFP balanced steady-state free precession

${ }^{a}$ Male:female ratio not provided in original publication

${ }^{\mathrm{b}}$ Age range not provided in original publication

measurements of the thickness of trabeculation per segment, with consequently different results.

\section{Cardiac valves and quantification of flow CMR acquisition parameters}

Prospectively and retrospectively electrocardiogram (ECG)-gated phase contrast (PC) CMR sequences are widely available. Prospectively-gated sequences use arrhythmia rejection and may be performed in a breath hold. Retrospectively gated techniques are mainly performed during free-breathing, often with higher spatial and temporal resolution compared to the breath hold techniques [67]. Four-dimensional flow-sensitive (4D Flow) PC CMR techniques have shown promising initial results, but 2D PC flow techniques remains the most commonly used approach in daily clinical practice [68]. In addition to PC-CMR, valve planimetry-using 
Table 29 Normal thickness of the trabeculated (noncompacted) left ventricular myocardium on short axis at enddiastole (in $\mathrm{mm}$ ) in the adult according to [56]

\begin{tabular}{llll}
\hline Level & Segment & Mean (median) & SD (IQR) \\
\hline Basal & 1 & 3.0 & $0,4.6$ \\
& 2 & 0 & \\
& 3 & 0 & \\
& 4 & 0 & $0,3.9$ \\
& 5 & 0 & $0,4.1$ \\
Mid-cavity & 6 & 0 & 2.8 \\
& 7 & 5.6 & \\
& 8 & 0 & $0,2.1$ \\
& 9 & 0 & 2.5 \\
Apical & 10 & 0 & 2.7 \\
& 11 & 4.2 & 2.7 \\
& 12 & 4.4 & $0,4.5$ \\
& 13 & 5.6 & 2.4 \\
\hline
\end{tabular}

According to the original publication $(n=120)$, data are presented as mean $\pm S D$ for normally distributed variables and as median (first, third interquartile ranges) for nonparametric variables; Segments: 1 = basal anterior, 2 =basal anteroseptal, $3=$ basal inferoseptal, $4=$ basal inferior, $5=$ basal inferolateral, $6=$ basal anterolateral, $7=$ mid anterior, $8=$ mid anteroseptal, $9=$ mid inferoseptal, $10=$ mid inferior, $11=$ mid inferolateral, $12=$ mid anterolateral, $13=$ apical anterior, $14=$ apical septal, $15=$ apical inferior, $16=$ apical lateral.

ECG-gated bSSFP CMR-can also be used to estimate stenosis or insufficiencies with good correlation to echocardiographic measurements [69].

Measurements of flow are most precise when (a) the imaging plane is positioned perpendicular to the vessel of interest and (b) the velocity encoded gradient echo $\left(\mathrm{V}_{\text {enc }}\right)$ is encoded in a through plane direction [70]. The slice thickness should be $\leq 7 \mathrm{~mm}$ to minimize partial volume effects. Compared to aortic or pulmonary artery flow evaluation, quantification of mitral or tricuspid valves is more challenging using PC-CMR due to through plane motion during the cardiac cycle [71].
The flow encoding velocity $\left(\mathrm{V}_{\text {enc }}\right)$ should be chosen close to the maximum expected flow velocity of the examined vessel for precise measurements. Setting the $V_{\text {enc }}$ below the peak velocity results in aliasing. For the normal aorta and main pulmonary artery, maximum velocities usually do not exceed 150 and $90 \mathrm{~cm} / \mathrm{s}$, respectively.

Adequate temporal resolution is necessary to avoid temporal flow averaging, especially for the evaluation of short, fast, and turbulent jets within a vessel (e.g. aortic stenosis). For clinical routine, 25-30 ms temporal resolution is sufficient. The minimum required spatial resolution is less than one third of the vessel diameter to avoid partial volume effects with the adjacent vessel wall and surrounding stationary tissues for small arteries [70].

\section{CMR analysis methods}

For data analysis, dedicated flow software should be used. Most of the currently available flow software tools offer semi-automatic vessel contouring, which needs to be carefully checked by the examiner.

The modified Bernoulli equation $\left(\Delta \mathrm{P}=4 \times \mathrm{V}_{\max }{ }^{2}\right)$ is commonly used for calculation of pressure gradients using PC-CMR across the pulmonary or aortic valve [72, 73].

Velocity measurements of valvular stenosis with high jet velocities may be inaccurate due to (A) partial volume effects in case of a small jet width and (B) limited temporal resolution compared to the high velocity of the jet. Measurements are further affected by signal loss due to the high velocity that may lead to phase shift errors and dephasing. Misalignment of the slice relative to the direction of the jet may also lead to an underestimation of the peak velocity [74].

Mitral valve flow velocities and deceleration times can be quantified for assessment of LV diastolic function, in a manner analogous to that used with transthoracic echocardiography (TTE). 2D PC derived trans-mitral flow velocities and deceleration times are strongly correlated

Table 30 Normal values for mass and volume of trabeculated (non-compacted) left ventricular myocardium in the adult measured on short axis images

\begin{tabular}{|c|c|c|c|c|c|c|c|}
\hline \multirow[t]{2}{*}{ Parameter } & \multirow[t]{2}{*}{ Technique } & \multicolumn{3}{|c|}{ Men } & \multicolumn{3}{|c|}{ Women } \\
\hline & & $n$ & Mean & SD & $n$ & Mean & SD \\
\hline $\begin{array}{l}\text { Trabeculation mass (mass of the trabeculated } \\
\text { [non-compacted] LV myocardium) per BSA (g/ } \\
\text { m2) from ref [12] }\end{array}$ & $\begin{array}{l}\text { Papillary muscles and } \\
\text { blood between trabeculae } \\
\text { excluded }\end{array}$ & 70 & 5.4 & 2.3 & 70 & 4.0 & 2.3 \\
\hline $\begin{array}{l}\text { Trabeculation volume (volume of the trabecu- } \\
\text { lated [non-compacted] LV myocardium) per } \\
\text { BSA (ml/m2) from ref [64] }\end{array}$ & $\begin{array}{l}\text { Blood between trabeculae } \\
\text { included, papillary muscles } \\
\text { excluded }\end{array}$ & 58 & 43.1 & 8.7 & 59 & 36.1 & 5.2 \\
\hline
\end{tabular}

$n$ number of study subjects, $S D$ standard deviation, $B S A$ body surface area 
Table 31 Normal values for the fractal dimension (FD) (unitless) of left ventricular trabeculation in the adult for different ethnicities

\begin{tabular}{|c|c|c|c|c|}
\hline Parameter & Ethnicity & $\mathrm{n}$ & Mean & SD \\
\hline Global FD from ref [59] & Black & 30 & 1.246 & 0.005 \\
\hline $\begin{array}{l}\text { Maximal apical FDa from } \\
\text { ref [59] }\end{array}$ & Black & 30 & 1.235 & 0.03 \\
\hline Global FD from ref [59] & White & 75 & 1.228 & 0.002 \\
\hline $\begin{array}{l}\text { Maximal apical FDa from } \\
\text { ref [59] }\end{array}$ & White & 75 & 1.253 & 0.025 \\
\hline Global FD from ref [66] & Singaporean Chinese & 180 & 1.205 & 0.031 \\
\hline $\begin{array}{l}\text { Maximal apical FD from } \\
\text { ref [66] }\end{array}$ & Singaporean Chinese & 180 & 0.278 & 0.045 \\
\hline
\end{tabular}

$n$ number of study subjects, SD standard deviation

${ }^{a}$ Measured for the apical third of the left ventricle

${ }^{b}$ Measured for the apical half of the left ventricle

with TTE derived parameters, but with a systematic underestimation [75].

\section{Demographic parameters}

To our knowledge, no comprehensive studies have been performed to investigate the association between age, gender and ethnicity and valvular flow or valve planimetry in normal healthy subjects based on PC-CMR. Two recent studies using 4D Flow CMR investigated the relationship of aortic flow velocity with age and gender, respectively [76, 77]. Callaghan et al. [76] compared measurements of mean peak systolic velocity obtained in the ascending aorta between 3 age groups and found a significant decrease with age. Garcia et al. [77] showed the mean aortic valve peak velocity was higher with greater age. In the study by Garcia et al. the differences in peak systolic velocity with gender were small and likely not clinically relevant [77].

\section{Studies included in this review}

There is good agreement between PC-CMR, bSSFP CMR planimetry, and echocardiography measurements. American Heart Association (AHA) criteria for grading valve stenosis or insufficiency is suggested [78, 79] (Table 33).
To our knowledge, there is no publication from a large study of normal reference values of trans-valvular flow and valve planimetry based on PC-CMR measurements.

Mitral valve flow parameters for determination of diastolic LV function are shown in Table 34.

Garcia, et al. [77] and Callaghan, et al. [76] have reported normal thoracic aorta flow parameters using 4D Flow CMR. Amongst other parameters, Garcia obtained measurements of peak systolic velocity where the transvalvular velocity reaches its maximum during peak systole (vena contracta region) (Fig. 8a) while Callaghan acquired measurement $6 \mathrm{~cm}$ proximal from the most cranial point of the aortic arch centerline in the ascending aorta (Fig. 8b). Normal values of peak aortic velocity are given in Tables 35 and 36.

\section{Normal aortic dimensions in the adult CMR acquisition parameters}

Three-dimensional contrast enhanced CMR angiography (CMRA) has gained broad acceptance and is widely used for the assessment and follow-up of thoracic aortic diameters in the clinical setting. The multi-planar reformation of CMRA images leads to an accurate measurement perpendicular to the lumen of the vessel. However, motion caused by pulsation leads to substantial blurring of the vessel contour at the level of the aortic root, hampering accurate diameter measurements [81]. The need of a contrast injection is another limitation for the use of this technique, particularly in patients who need multiple follow up examinations and in population based study settings [82]. Alternatively non-contrast techniques such as an ECG- and respiratory-gated gadolinium-enhanced CMRA or 3D bSSFP sequence can be applied, enabling accurate measurements of aortic diameters including the aortic root [82]. However, due to the long acquisition times or lack of sequence availability, these methods may not be widely applied [81]. The magnitude image of PC CMR has also been used to measure diameters of the aorta [83]. Black blood techniques are used for a more detailed assessment of the aortic wall [84].

In $2 \mathrm{D}$ acquisitions, the imaging plane needs to be acquired correctly at the time of the scan; thus, any alterations in the

Table 32 Normal values for the fractal dimension (FD) (unitless) of left ventricular trabeculation in the adult stratified by sex and body mass index (BMI) according to reference [62]

\begin{tabular}{|c|c|c|c|c|c|c|c|c|c|}
\hline \multirow[t]{2}{*}{ Parameter } & \multicolumn{3}{|c|}{$\mathrm{BMI} \geq 30 \mathrm{~kg} / \mathrm{m}^{2}($ mean $\pm \mathrm{SD})$} & \multicolumn{3}{|c|}{$\mathrm{BMI} \geq 25$ to $<30 \mathrm{~kg} / \mathrm{m}^{2}($ mean $\pm \mathrm{SD})$} & \multicolumn{3}{|c|}{$\mathrm{BMI}<25 \mathrm{~kg} / \mathrm{m}^{2}($ mean $\pm \mathrm{SD})$} \\
\hline & All $(n=163)$ & Men $(n=71)$ & $\begin{array}{l}\text { Women } \\
(n=92)\end{array}$ & All $(n=206)$ & $\begin{array}{l}\text { Men } \\
(n=108)\end{array}$ & $\begin{array}{l}\text { Women } \\
(n=98)\end{array}$ & All $(n=235)$ & $\begin{array}{l}\text { Men } \\
(n=100)\end{array}$ & $\begin{array}{l}\text { Women } \\
(n=135)\end{array}$ \\
\hline $\begin{array}{l}\text { Max. apical } \\
\text { FDa }\end{array}$ & $1.203 \pm 0.06$ & $1.212 \pm 0.07$ & $1.196 \pm 0.06$ & $1.194 \pm 0.06$ & $1.197 \pm 0.05$ & $1.190 \pm 0.07$ & $1.169 \pm 0.07$ & $1.177 \pm 0.06$ & $1.162 \pm 0.05$ \\
\hline
\end{tabular}

$n$ number of study subjects, $B M I$ body mass index, SD standard deviation, Max. maximal

${ }^{\text {a }}$ Measured for the apical half of the left ventricle 
Table 33 Stages of valvular heart disease in the adult. adapted from echocardiography according to references $[78,79]$

\begin{tabular}{|c|c|c|c|}
\hline \multirow[t]{2}{*}{ Valve disease } & \multirow[t]{2}{*}{ Parameter } & \multicolumn{2}{|l|}{ Stage } \\
\hline & & Progressive & Severe \\
\hline \multirow[t]{3}{*}{ Aortic stenosis } & Maximum velocity $(\mathrm{m} / \mathrm{s})$ & $\begin{array}{l}\text { Mild: } 2.0-2.9 \\
\text { Moderate: } 3.0-3.9\end{array}$ & $\begin{array}{l}\text { Severe: } \geq 4 \\
\text { Very severe: } \geq 5 \\
\text { Low-flow/low- } \\
\text { gradient: }<4 \mathrm{~m} / \mathrm{s} \\
\text { (at rest) }\end{array}$ \\
\hline & Orifice area $\left(\mathrm{cm}^{2}\right)$ & & $\leq 1.0$ \\
\hline & Orifice area $/ \mathrm{BSA}\left(\mathrm{cm}^{2} / \mathrm{m}^{2}\right)$ & & $\leq 0.6$ \\
\hline \multirow[t]{3}{*}{ Aortic regurgitation } & Regurgitant volume (ml/beat) & $\begin{array}{l}\text { Mild: }<30 \\
\text { Moderate: } 30-59\end{array}$ & $\geq 60$ \\
\hline & Regurgitant fraction (\%) & $\begin{array}{l}\text { Mild: }<30 \\
\text { Moderate: } 30-49\end{array}$ & $\geq 50$ \\
\hline & Effective regurgitant orifice $\left(\mathrm{cm}^{2}\right)$ & $\begin{array}{l}\text { Mild: }<0.10 \\
\text { Moderate } 0.10-0.29\end{array}$ & $\geq 0.30$ \\
\hline \multirow[t]{2}{*}{ Mitral stenosis } & Transmitral flow velocity (m/s) & Increased & \\
\hline & Orifice area $\left(\mathrm{cm}^{2}\right)$ & $>1.5$ & $\begin{array}{l}\text { Severe: } \leq 1.5 \\
\text { Very severe: } \leq 1.0\end{array}$ \\
\hline \multirow[t]{3}{*}{ Primary mitral regurgitation } & Regurgitant volume (ml/beat) & $<60$ & $\geq 60$ \\
\hline & Regurgitant fraction (\%) & $<50$ & $\geq 50$ \\
\hline & Effective regurgitant orifice $\left(\mathrm{cm}^{2}\right)$ & $<0.40$ & $\geq 0.40$ \\
\hline \multirow[t]{3}{*}{ Secondary mitral regurgitation } & Regurgitant volume (ml/beat) & $<60$ & $\geq 60$ \\
\hline & Regurgitant fraction (\%) & $<50$ & $\geq 50$ \\
\hline & Effective regurgitant orifice $\left(\mathrm{cm}^{2}\right)$ & $<0.40$ & $\geq 0.40$ \\
\hline Pulmonic stenosis & Peak velocity (m/s) & & $>4$ \\
\hline Tricuspid stenosis & Orifice area $\left(\mathrm{cm}^{2}\right)$ & & $<1.0$ \\
\hline
\end{tabular}

$B S A$ body surface area

Table 34 Mitral valve flow for determination of diastolic left ventricular function according to reference [80]

\begin{tabular}{lcllll}
\hline Parameter & Normal & $\begin{array}{l}\text { Type 1 (Impaired } \\
\text { relaxation) }\end{array}$ & Type 2 (Pseudonormal) & $\begin{array}{l}\text { Type 3 (Restrictive, partially } \\
\text { reversible) }\end{array}$ & $\begin{array}{l}\text { Type } 3 \\
\text { (Restrictive, } \\
\text { fixed) }\end{array}$ \\
\hline MDT (ms) & $150-220$ & Increased & Normal & Decreased & Decreased \\
E/A ratio & $1-2$ & $<1$ & $1-2$ & $>2$ & $>2$ \\
\hline
\end{tabular}

$M D T$ mitral deceleration time, E/A ratio ratio of the mitral early $(\mathrm{E})$ and atrial $(\mathrm{A})$ components of the mitral inflow velocity profile

imaging plane due to breath-holding or patient motion will result in variability of measurements. Through plane motion during the cardiac cycle can be minimized with ECG gating [82].

Potthast and colleagues compared the diameter of the ascending aorta obtained by different CMR sequences to ECG-triggered computed tomography angiography (CTA) as the standard of reference. They reported that ECG-gated navigator triggered 3D bSSFP sequence showed the best agreement with CTA [82].

\section{CMR analysis methods}

Beside the sequence type, imaging plane and cardiac phase (systole versus diastole), it is important to identify the anatomic locations of diameter measurements of the thoracic aorta (Fig. 9).

The sagittal oblique view of the LV outflow tract was used for measuring diameter at the level of the aortic annulus, the aortic sinus, and the sinotubular junction (Fig. 10) [11, 85, 86]. Axial cross sectional images at predefined anatomic levels were used for measuring the ascending and descending aorta [86] as well as cuspcommissure and cusp-cusp diameters at the level of the aortic sinus [85] (Fig. 11).

Luminal or outer to outer diameter of the aorta may be measured. The current SCMR guidelines on image post-processing recommend measurement of the outer contour in dilatation while measurements of the inner 


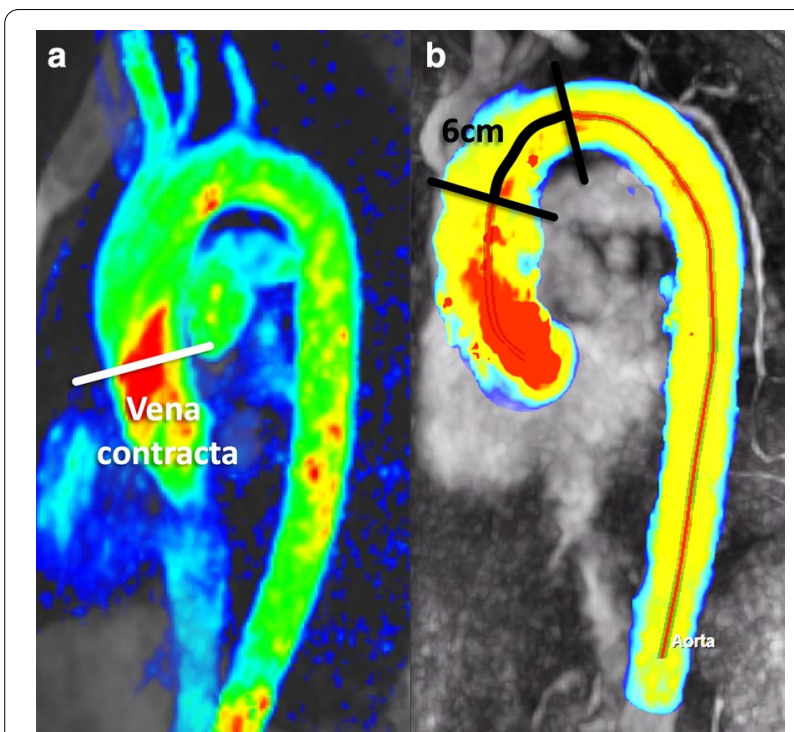

Fig. 8 Images of a 4D flow sequence illustrating sites of measurement of peak systolic velocity. According to reference [77] measurements were obtained where the transvalvular velocity reaches its maximum during peak systole (vena contracta region) (a). In reference [76] peak systolic velocity was obtained in the ascending aorta $6 \mathrm{~cm}$ proximal from the most cranial point of the aortic arch centerline (b)

contour should be obtained in the setting of stenosis [9]. In the tables below, the method is specified.

\section{Demographic parameters}

In the MESA, a large population based study, the diameter of the ascending aorta has been shown to be larger in men compared to women even after adjustment for BSA
[83]. In a publication by Le et al., however, the gender difference in diameters did not persist after normalization to BSA [11].

Several studies have shown an increase in aortic diameter with age $[11,83,85,86]$. The association of age with aortic diameter was more marked in the ascending aorta compared to the descending thoracic and abdominal aorta, respectively [87, 88]. Further, age-related changes of the geometry of the thoracic aorta have been described. Age-related changes include increasing length of the ascending aorta and decreasing curvature of the aortic arch $[89,90]$.

In the MESA study, there were small differences in normal aortic diameter for Chinese and African American participants compared to Caucasians. These differences were small however relative to measurement error and reproducibility and therefore may not be clinically relevant [83].

\section{Studies included in this review}

Studies with normal values of aortic diameters based on measurements obtained in studies with 40 or more healthy subjects per gender have been included in this review (Table 37). There are five major publications regarding CMR-based measurements of the thoracic aorta in adults [11, 83-86]. There is substantial difference between the studies with respect to CMR sequences (cine bSSFP, PC CMRA and 3D-T1-black blood volume isotropic turbo spin echo acquisition), acquisition/ measurement plane (cross sectional versus LV outflow tract view), measurement technique (luminal versus total diameter and area, respectively) and measurement sites of the aorta. Therefore, results of most studies are presented separately (Tables 38, 39, 40, 41). Details of image

Table 35 Normal mean peak systolic velocity of the ascending aorta by 4D-flow for different age groups according to reference [76]

\begin{tabular}{|c|c|c|c|c|c|c|c|c|c|c|c|c|}
\hline \multirow[t]{2}{*}{ Parameter } & \multicolumn{4}{|c|}{ 18-33 years } & \multicolumn{4}{|c|}{$34-60$ years } & \multicolumn{4}{|c|}{$>60$ years } \\
\hline & $\mathbf{n}$ & Mean & SD & ${\mathrm{LL}-U L^{\mathrm{a}}}$ & $\mathbf{n}$ & Mean & SD & ${\mathrm{LL}-U L^{\mathrm{a}}}$ & $n$ & Mean & SD & $\mathrm{LL}_{-} \mathrm{UL}^{\mathrm{a}}$ \\
\hline Velocity $(\mathrm{cm} / \mathrm{s})$ & 64 & 66 & 15 & $36-96$ & 116 & 51 & 13 & $25-77$ & 67 & 35 & 12 & $11-59$ \\
\hline
\end{tabular}

Measured $6 \mathrm{~cm}$ proximal from the most cranial point of the aortic arch centerline (Fig. $8 \mathrm{~b}$ )

$n$ number of study subjects, SD standard deviation, LL lower limit, UL upper limit

a Calculated as mean $\pm 2 * S D$

Table 36 Normal mean aortic valve peak velocity by 4D-flow CMR for men and women according to reference [77]

\begin{tabular}{|c|c|c|c|c|c|c|c|c|}
\hline \multirow[t]{2}{*}{ Parameter } & \multicolumn{4}{|c|}{ Men } & \multicolumn{4}{|c|}{ Women } \\
\hline & $\mathrm{n}$ & Mean & SD & $\overline{L_{L}-U L^{a}}$ & $n$ & mean & SD & LL-UL ${ }^{a}$ \\
\hline Velocity (m/s) & 57 & 1.3 & 0.3 & $0.8-1.8$ & 41 & 1.2 & 0.2 & $0.8-1.6$ \\
\hline
\end{tabular}

Measured where the transvalvular velocity reaches its maximum during peak systole (Fig. 8a)

$n$ number of study subjects, SD standard deviation, LL lower limit, UL upper limit

${ }^{\text {a }}$ Calculated as mean $\pm 2 * S D$ 


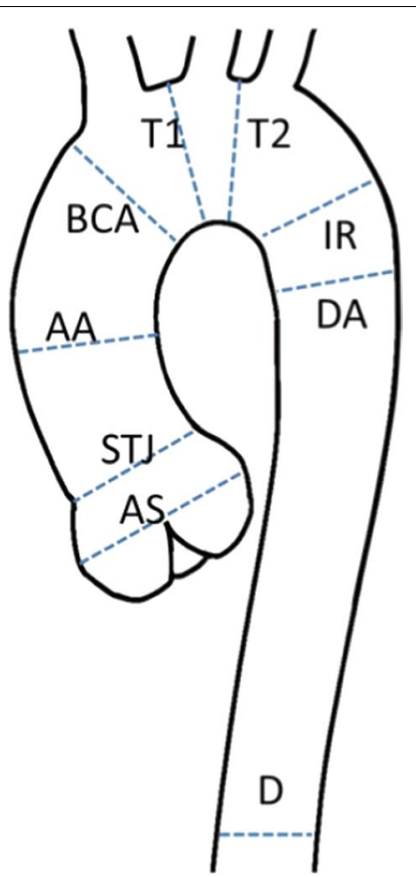

Fig. 9 Sites of measurement of the thoracic aorta. AS aortic sinus, STJ sinotubular junction, $A A$ ascending aorta, $B C A$ proximal to the origin of the brachiocephalic artery, $T 1$ between the origin of the brachiocephalic artery and the left common carotid artery, $T 2$ between the origin of the left common carotid artery and the left subclavian artery, IR isthmic region, $D A$ descending aorta, $D$ thoracoabdominal aorta at the level of the diaphragm

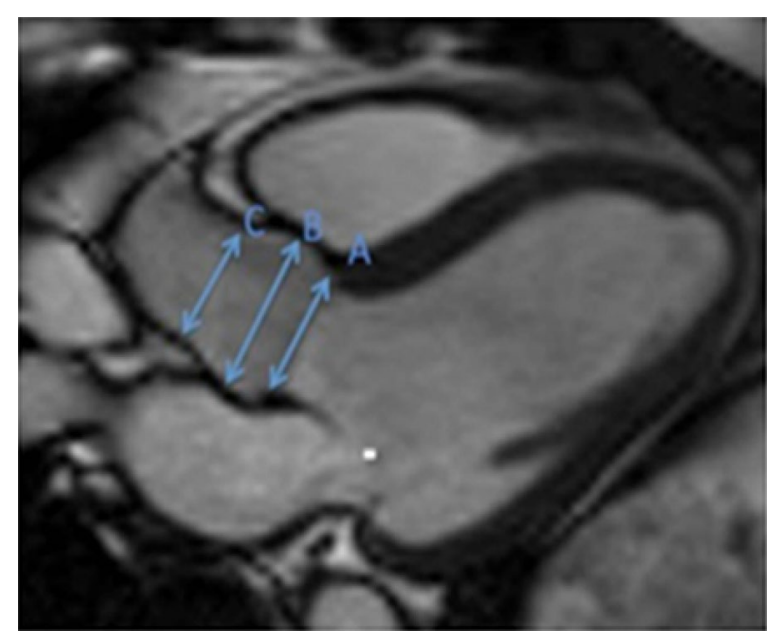

Fig. 10 Measurements of luminal diameters of the aortic annulus (a), the aortic sinus (b) and the sinotubular junction (c) obtained on a steady-state free precession left ventricular outflow tract view at diastole according to reference [86]

acquisition and measurement technique of each study can be found in Table 37 and are described in the footnote of each table.

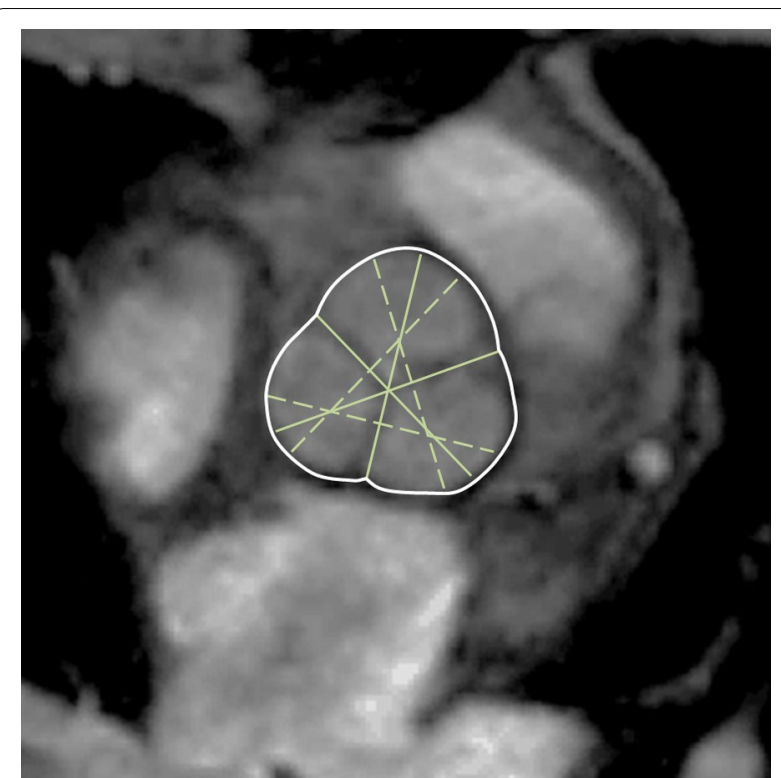

Fig. 11 Cusp-commissure (continuous lines) and cusp-cusp (dashed-lines) measurements at the level of the aortic sinus according to reference [85]

Weighted means were calculated based on the values of the diameter of the aortic root obtained on the 3D bSSFP sequence in LVOT view published by Burman, Davis and Le (Table 42) $[11,85,86]$.

\section{Normal aortic dimensions in children CMR acquisition parameters}

There is currently no consensus regarding the optimal CMR sequence to measure aortic diameters and areas in children. In three major publications documenting aortic dimensions in children (Table 43), measurements were obtained with three-dimensional contrast enhanced CMRA [91], gradient echo images [92] and phase contrast cine images [93].

\section{CMR analysis methods}

To minimize errors in measurement of aorta size, multiplanar reformation should be used to make doubleoblique measurements perpendicular to the centerline of the course of the vessel. Kaiser et al. demonstrated that aortic diameter measurements vary slightly based on plane orientation, with a mean difference between measurements on cross-sectional and longitudinal images of $0.16 \mathrm{~mm}$ and a coefficient of variability of $2.1 \%$ [91].

Aorta measurements should also be made in a consistent manner with respect to the wall of the aorta-outer wall to outer wall, leading edge to leading edge, or luminal diameter. Kutty et al. indicated that in their study measurements were made from outer wall to outer wall 
Table 37 References, normal aortic diameters, area and wall thickness in adults

\begin{tabular}{|c|c|c|c|}
\hline First author, year & CMR technique & $\mathrm{n}$, male:female & Age range (years) \\
\hline Burman, 2008 [85] & $\begin{array}{l}\text { 1.5 T, cine bSSFP, luminal diameter at systole and diastole, average of } 3 \text { cusp-commissure } \\
\text { and } 3 \text { cusp-cusp diameters, respectively on cross-sectional images of the aortic sinus and } \\
\text { diameter of the aortic sinus on the sagittal LVOT plane }\end{array}$ & $60: 60$ & $20-80$ \\
\hline Davis, 2014 [86] & $\begin{array}{l}\text { 1.5 T, cine bSSFP, maximal luminal diameter at diastole, diameters calculated based on } \\
\text { measurements of the area at } 3 \text { levels (ascending aorta, proximal and distal descending } \\
\text { aorta) of the aorta on cross-sectional images and diameters at } 3 \text { levels (annulus, sinus, } \\
\text { sinotubular junction) of the aortic root measured on the sagittal LVOT plane }\end{array}$ & $208: 239$ & 19-70 \\
\hline Turkbey, 2014 [83] & $\begin{array}{l}1.5 \mathrm{~T} \text {, luminal diameter of the ascending aorta measured on the magnitude image of a } \\
\text { phase contrast sequence }\end{array}$ & $770: 842$ & $45-84$ \\
\hline Eikendal, 2016 [84] & $\begin{array}{l}\text { 3T, fat suppressed 3D-T1-black blood VISTA acquired sagittal of the descending aorta, } \\
\text { luminal and total vessel diameter and area, calculated average diameter, luminal and total } \\
\text { vessel area, vessel wall area and thickness of the proximal to distal descending aorta after } \\
\text { manual tracing of the luminal and outer aortic wall on axial reformatted images }\end{array}$ & $59: 65$ & $25-35$ \\
\hline Le, 2016 [11] & $\begin{array}{l}3 \text { T, cine bSSFP, luminal diameter of the aortic annulus, sinus and sinotubular junction at } \\
\text { diastole measured on the sagittal LVOT plane }\end{array}$ & $91: 89$ & $20-69$ \\
\hline
\end{tabular}

$n$ number of study subjects, bSSFP balanced steady-state free precession, LVOT left ventricular outflow tract, VISTA volume isotropic turbo spin echo acquisition

Table 38 Absolute and indexed (to BSA) normal values of aortic sinus luminal diameters and area for men and women at systole and diastole according to [85]

\begin{tabular}{|c|c|c|c|c|}
\hline \multirow[t]{2}{*}{ Parameter } & \multicolumn{2}{|c|}{ Men $(n=60)\left[\right.$ mean \pm SD $\left.(L L-U L){ }^{a}\right]$} & \multicolumn{2}{|c|}{ Women $(n=60)\left[\right.$ mean \pm SD (LL-UL) $\left.{ }^{a}\right]$} \\
\hline & Systolic & Diastolic & Systolic & Diastolic \\
\hline Aortic sinus diameter (cusp-commissure) (mm) & $34 \pm 3(27-40)$ & $32 \pm 4(25-39)$ & $30 \pm 3(25-35)$ & $28 \pm 3(23-34)$ \\
\hline Aortic sinus diameter (cusp-commissure)/BSA (mm/m²) & $17 \pm 2(14-20)$ & $16 \pm 2(13-20)$ & $18 \pm 2(14-21)$ & $17 \pm 2(13-20)$ \\
\hline Aortic sinus diameter (cusp-cusp) (mm) & $36 \pm 4(28-44)$ & $35 \pm 4(27-43)$ & $32 \pm 3(26-38)$ & $31 \pm 3(24-37)$ \\
\hline Aortic sinus diameter (cusp-cusp)/BSA (mm/ $\left.\mathrm{m}^{2}\right)$ & $18 \pm 2(14-22)$ & $18 \pm 2(14-22)$ & $19 \pm 2(15-23)$ & $18 \pm 2(14-22)$ \\
\hline Aortic sinus area $\left(\mathrm{cm}^{2}\right)$ & $9.2 \pm 2.1(5.0-13.4)$ & $8.4 \pm 2.0(4.4-12.4)$ & $7.1 \pm 1.4(4.3-9.9)$ & $6.5 \pm 1.3(3.9-9.1)$ \\
\hline Aortic sinus area/BSA $\left(\mathrm{cm}^{2} / \mathrm{m}^{2}\right)$ & $4.6 \pm 1.0(2.6-6.6)$ & $4.2 \pm 0.9(2.4-6.0)$ & $4.2 \pm 0.8(2.6-5.8)$ & $3.8 \pm 0.8(2.2-5.4)$ \\
\hline
\end{tabular}

Values obtained as the average of 3 cusp-commissure and 3 cusp-cusp diameters, respectively measured on cross-sectional bSSFP images of the aortic sinus (Fig. 11) $n$ number of study subjects, SD standard deviation, LL lower limit, UL upper limit, BSA body surface area

${ }^{\text {a }}$ Calculated as mean $\pm 2 * S D$

Table 39 Normal values of the thoracic aortic luminal diameters for men and women measured at diastole on bSSFP images according to [86]

\begin{tabular}{lcc}
\hline Level & $\begin{array}{l}\text { Men }(\mathbf{n}=\mathbf{2 0 8}) \\
\text { Mean } \pm \text { SD (LL-UL) }\end{array}$ & $\begin{array}{c}\text { Women }(\mathbf{n}=\mathbf{2 3 9}) \\
\text { Mean } \pm \mathbf{S D}(\mathbf{L L}-\mathbf{U L})^{\mathbf{a}}\end{array}$ \\
\hline Ascending aorta diameter $(\mathrm{mm})$ & $27 \pm 4(19-34)$ & $26 \pm 4(18-33)$ \\
Proximal descending aorta diameter $(\mathrm{mm})$ & $21 \pm 3(15-26)$ & $19 \pm 2(15-23)$ \\
Distal descending aorta diameter $(\mathrm{mm})$ & $18 \pm 3(13-23)$ & $16 \pm 2(12-20)$ \\
\hline
\end{tabular}

Measurements obtained on cross-sectional bSSFP images of the aorta

bSSFP balanced steady-state free precession, $n$ number of study subjects, SD standard deviation, $L L$ lower limit, UL upper limit

${ }^{a}$ Calculated as mean $\pm 2 * S D$

[93]. Kaiser et al. and Voges et al. did not provide details on how measurements were made in this regard $[91,92]$.

\section{Demographic parameters}

Aortic diameters vary by BSA $[91,93]$ but do not show sex differences in children [92, 93]. Aortic area has not been shown to be dependent upon sex differences either [92].

\section{Studies included in this review}

Reference ranges for parameters measured in children are frequently presented in $\mathrm{z}$-scores and reference curves using the LMS method as described under the LV/RV parameter section in children above.

There are three publications of systematic evaluation of aortic dimensions (diameter and/or area) in children 
Table 40 Absolute and BSA indexed normal values of ascending aortic luminal diameter for men and women of different age categories measured on phase contrast images according to [83]

\begin{tabular}{lll}
\hline Age (years) & $\begin{array}{l}\text { Men }(\mathbf{n}=\mathbf{7 7 0}) \\
\text { Median } \\
\text { (5th-95th } \\
\text { percentile) }\end{array}$ & $\begin{array}{l}\text { Women } \\
\mathbf{(}=\mathbf{8 4 2}) \\
\text { Median } \\
\text { (5th-95th } \\
\text { percentile) }\end{array}$ \\
\hline Absolute values (mm) & & \\
$45-54$ & $32(27-37)$ & $29(25-34)$ \\
$55-64$ & $33(28-41)$ & $30(26-36)$ \\
$65-74$ & $34(29-41)$ & $31(26-36)$ \\
$75-84$ & $35(29-41)$ & $31(27-37)$ \\
Values indexed to BSA (mm/m²) & & $17(14-21)$ \\
$45-54$ & $16(13-20)$ & $18(15-22)$ \\
$55-64$ & $17(14-21)$ & $18(15-22)$ \\
$65-74$ & $18(14-22)$ & $20(15-28)$ \\
$75-84$ & $19(15-23)$ & \\
\hline
\end{tabular}

$n$ number of study subjects, $B S A$ body surface area

Table 41 Normal values of descending thoracic aortic diameter, area and wall thickness for young men and women (25-35 years) according to [84]

\begin{tabular}{lcc}
\hline Parameter & $\begin{array}{l}\text { Men }(\mathbf{n}=\mathbf{5 9}) \\
\text { Median } \\
\text { (10th-90th } \\
\text { percentile) }\end{array}$ & $\begin{array}{l}\text { Women }(\mathbf{n}=65) \\
\text { Median } \\
\text { (10th-90th } \\
\text { percentile) }\end{array}$ \\
\hline $\begin{array}{l}\text { Luminal diameter descending } \\
\text { aorta (mm) }\end{array}$ & $19(17-21)$ & $17(16-19)$ \\
$\begin{array}{l}\text { Total diameter descending aorta } \\
(\mathrm{mm})\end{array}$ & $22(20-24)$ & $20(19-22)$ \\
$\begin{array}{l}\text { Luminal area descending aorta } \\
\left(\mathrm{cm}^{2}\right)\end{array}$ & $2.9(2.2-3.5)$ & $2.3(2.0-2.8)$ \\
$\begin{array}{l}\text { Total area descending aorta }\left(\mathrm{cm}^{2}\right) \\
\text { Wall area descending aorta }\left(\mathrm{cm}^{2}\right)\end{array}$ & $3.9(3.1-4.6)$ & $3.3(2.8-3.9)$ \\
$\begin{array}{l}\text { Wall thickness descending aorta } \\
(\mathrm{mm})\end{array}$ & $1.5(1.4-1.8)$ & $1.0(0.8-1.1)$ \\
& & $1.5(1.4-1.9)$
\end{tabular}

Measurements obtained on axial reformatted images of a fat suppressed 3-dimensional-T1-black blood VISTA (volume isotropic turbo spin echo acquisition) sequence. Calculated average vessel diameter and area as well as wall thickness and wall area of the descending aorta.

$n$ number of study subjects

that vary by CMR-technique, measurement technique and data presentation (Table 43).

In this review we present (a) LMS parameters to calculate z-scores for aortic cross-section area based on reference [92] (Tables 44, 45) (b) regression equations of normal aortic diameters measured at 9 different sites based on [91] (Table 46) and (c) normal areas of the ascending aorta from [93] (Table 47).
Due to the differences in acquisition sequences, measurement techniques, and presentation of results, weighted mean values are not presented.

\section{Normal aortic distensibilityand pulse wave velocity (PWV) in adults \\ CMR acquisition parameters}

Pulse wave velocity (PWV) calculations using a velocity-encoded CMR with phase contrast sequences allow accurate assessment of aortic systolic flow wave and the blood flow velocity. The sequence should be acquired at the level of the bifurcation of the pulmonary trunk, perpendicular to both, the ascending and descending aorta. The distance between two aortic locations (aortic length) can be estimated from axial and coronal cine breath hold bSSFP sequences covering the whole aortic arch [94]. Alternatively, sagittal oblique views of the aortic arch can be acquired e.g. using a black blood spin echo sequence [88].

Another parameter of aortic stiffness is aortic distensibility. The cross sectional aortic area at different phases of the cardiac cycle is measured using ECG-gated bSSFP cine imaging to assess aortic distensibility by CMR. Modulus images of cine phase contrast CMR can be used as well [95].

\section{CMR analysis methods}

PWV is the most validated method to quantify arterial stiffness using CMR. PWV is calculated by measuring the pulse transit time of the flow curves $(\Delta t)$ and the distance (D) between the ascending and descending aortic locations of the phase contrast acquisition [88]: Aortic $\mathrm{PWV}=\mathrm{D} / \Delta \mathrm{t}$ (Fig. 12).

PWV increases with stiffening of arteries since the stiffened artery conducts the pulse wave faster compared to more distensible arteries.

Aortic distensibility is calculated with the fallowing formula after measuring the minimum and maximum aortic cross sectional area [96]:

$$
\begin{aligned}
\text { Aortic distensibility }= & (\text { minimal area }- \text { maximal area }) / \\
& (\text { minimal area } \times \Delta \mathrm{P} \times 1000) \\
& \text { where } \Delta \mathrm{P} \text { is the pulse pressure in } \mathrm{mmHg} .
\end{aligned}
$$

\section{Demographic parameters}

Greater ascending aorta diameter and changes in aortic arch geometry with greater age was associated with increased regional stiffness of the aorta, especially of the ascending portion. The relationship of 
Table 42 Normal diameters of the aortic root for men and women measured on sagittal left ventricular outflow tract bSSFP

\begin{tabular}{|c|c|c|c|c|c|c|c|c|}
\hline \multirow[t]{2}{*}{ Parameter } & \multicolumn{4}{|l|}{ Men } & \multicolumn{4}{|c|}{ Women } \\
\hline & $n$ & Mean $_{p}$ & $S D_{p}$ & $\overline{L L-U L^{e}}$ & $n$ & Mean $_{p}$ & $S D_{p}$ & LL-UL ${ }^{\mathrm{e}}$ \\
\hline Aortic annulus diameter $(\mathrm{mm})^{\mathrm{a}}$ & 299 & 23 & 5 & $14-33$ & 328 & 20 & 3 & $14-27$ \\
\hline Aortic annulus diameter/BSA $\left(\mathrm{mm} / \mathrm{m}^{2}\right)^{\mathrm{b}}$ & 91 & 12 & 1 & $10-14$ & 89 & 12 & 1 & $10-14$ \\
\hline Aortic sinus diameter $(\mathrm{mm})^{c}$ & 359 & 32 & 6 & $19-45$ & 388 & 28 & 5 & $17-38$ \\
\hline Aortic sinus diameter/BSA $\left(\mathrm{ml} / \mathrm{m}^{2}\right)^{d}$ & 151 & 17 & 2 & $13-21$ & 149 & 17 & 2 & $13-21$ \\
\hline Sinotubular junction diameter $(\mathrm{mm})^{\mathrm{a}}$ & 299 & 25 & 6 & $12-38$ & 328 & 21 & 5 & $12-31$ \\
\hline Sinotubular junction diameter/BSA $\left(\mathrm{mm} / \mathrm{m}^{2}\right)^{b}$ & 91 & 13 & 2 & $10-17$ & 89 & 14 & 2 & $10-17$ \\
\hline
\end{tabular}

Measurements obtained as shown in Fig. 10.

$n$ number of study subjects included in the weighted mean values, $b S S F P$ balanced steady-state free precession, mean ${ }_{p}$ pooled weighted mean, $S D_{p}$ pooled standard deviation, LL lower limit, UL upper limit, BSA body surface area

a Pooled weighted values from references $[11,86]$

${ }^{b}$ Values from reference [11]

' Pooled weighted values from references $[11,85,86]$

d Pooled weighted values from references $[11,85]$

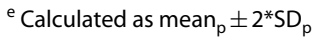

Table 43 References, normal aortic dimensions in children

\begin{tabular}{|c|c|c|c|}
\hline First author, year & CMR technique & n, male:female & $\begin{array}{l}\text { Age } \\
\text { range } \\
\text { (years) }\end{array}$ \\
\hline Kaiser, 2008 [91] & $\begin{array}{l}1.5 \mathrm{~T} \text {; contrast enhanced CMRA; shortest diameter measured on cross-sectional reformatted images at } \\
9 \text { locations }\end{array}$ & $30: 23$ & $2-20$ \\
\hline Kutty, 2012 [93] & $\begin{array}{l}1.5 \mathrm{~T} \text {; magnitude image of a through-plane free-breathing phase contrast sequence; cross-sectional } \\
\text { area calculated based on measurement of the maximal external aortic diameter perpendicular to the } \\
\text { vessel and perpendicular to the maximal diameter in systole } 1 \text { to } 2 \mathrm{~cm} \text { distal to the sinotubular junc- } \\
\text { tion }\end{array}$ & $55: 50$ & $4-20$ \\
\hline Voges, 2012 [92] & $\begin{array}{l}3 \mathrm{~T} \text {; cross sectional cine gradient echo images acquired at } 4 \text { positions perpendicular to the aortic axis, } \\
\text { measurements obtained at maximal distension of the aorta }\end{array}$ & $30: 41$ & $2-28$ \\
\hline
\end{tabular}

$n$ number of study subject

age with measures of aortic stiffness is non-linear and the decrease of aortic distensibility occurs particularly before the fifth decade of life [88]. Males have stiffer aortas compared to females [97].

\section{Studies included in this review}

Two studies with a total sample size of more than 40 subjects reported reference ranges for PWV and/or distensibility in healthy subjects (Table 48): Kim et al. present reference ranges for PWV and distensibility for a cohort of 124 healthy Asian subjects [98]. Since both parameters have been shown to be highly age dependent, reference ranges are given per age decile according to the original publication [98]. However, sample size per decile was small (between 21 and 28 subjects) and standard deviations are relatively large (Tables 49,50 ). In the study by Eikendal et al. reference values for PWV in young (25-35 years) healthy subjects are given (Table 51) [84].
With respect to PWV, in this review we present reference ranges for the distance between the ascending and the proximal descending thoracic aorta. This range is frequently measured since measurements at both locations can be obtained simultaneously on a single $2 \mathrm{D}$ acquisition at the level of the bifurcation of the pulmonary artery. PWV for other distances (ascending to distal descending aorta and total PWV) can be found in the original publications [84, 98]. In addition to the ascending and proximal descending thoracic aorta, distensibility for the distal descending and the total aorta is presented in the original publication by Kim et al. [98].

\section{Normal aortic distensibility and pulse wave velocity (PWV) in children \\ CMR acquisition parameters}

In the only publication of aortic distensibility and PWV by CMR in children, distensibility was obtained on gradient echo cine images and pulse wave velocity was measured on phase-contrast cine CMR [92]. 
Table 44 LMS parameters to calculate z-scores for aortic cross-sectional area relative to age for boys according to reference [92]

\begin{tabular}{|c|c|c|c|c|c|c|c|c|c|c|c|c|}
\hline \multirow[t]{2}{*}{$\mathrm{Age}^{\mathrm{a}}$} & \multicolumn{3}{|c|}{ Ascending aorta } & \multicolumn{3}{|c|}{ Aortic arch } & \multicolumn{3}{|c|}{ Aortic isthmus } & \multicolumn{3}{|c|}{ Descending aorta ${ }^{b}$} \\
\hline & $\mathbf{L}$ & $M$ & $S$ & $\mathbf{L}$ & $M$ & $S$ & $\mathbf{L}$ & $M$ & $S$ & L & M & $S$ \\
\hline$<1$ & 0.3091 & 91.5360 & 0.1207 & 0.8668 & 80.1737 & 0.1898 & 0.1267 & 53.0050 & 0.1987 & 1.5823 & 44.6080 & 0.1100 \\
\hline 1 & 0.3091 & 120.6960 & 0.1274 & 0.8668 & 101.7001 & 0.1897 & 0.1267 & 68.7198 & 0.1974 & 1.5823 & 57.0317 & 0.1115 \\
\hline 2 & 0.3091 & 149.8560 & 0.1341 & 0.8668 & 123.2265 & 0.1895 & 0.1267 & 84.4347 & 0.1960 & 1.5823 & 69.4554 & .1129 \\
\hline 3 & 0.3091 & 179.0160 & 0.1408 & 0.8668 & 144.7529 & 0.1894 & 0.1267 & 100.1495 & 0.1946 & 1.5823 & 81.8791 & 0.1143 \\
\hline 4 & 0.3091 & 208.1812 & 0.1475 & 0.8668 & 166.2791 & 0.1893 & 0.1267 & 115.8653 & 0.1932 & 1.5823 & 94.3035 & 0.1158 \\
\hline 5 & 0.3091 & 238.3791 & 0.1542 & 0.8668 & 187.7555 & 0.1891 & 0.1267 & 131.7743 & 0.1918 & 1.5823 & 106.8833 & 0.1172 \\
\hline 6 & 0.3091 & 272.8715 & 0.1604 & 0.8668 & 208.8732 & 0.1890 & 0.1267 & 148.2790 & 0.1904 & 1.5823 & 119.9057 & 0.1186 \\
\hline 7 & 0.3091 & 311.2493 & 0.1660 & 0.8668 & 229.2411 & 0.1888 & 0.1267 & 164.9648 & 0.1891 & 1.5823 & 133.0488 & 0.1201 \\
\hline 8 & 0.3091 & 346.8686 & 0.1707 & 0.8668 & 248.8676 & 0.1887 & 0.1267 & 180.7624 & 0.1877 & 1.5823 & 145.5984 & 0.1215 \\
\hline 9 & 0.3091 & 380.0230 & 0.1748 & 0.8668 & 268.0557 & 0.1886 & 0.1267 & 195.7825 & 0.1863 & 1.5823 & 157.5124 & 0.1229 \\
\hline 10 & 0.3091 & 413.8181 & 0.1782 & 0.8668 & 287.2956 & 0.1884 & 0.1267 & 210.6578 & 0.1849 & 1.5823 & 169.3366 & 0.1244 \\
\hline 11 & 0.3091 & 446.7220 & 0.1812 & 0.8668 & 306.7317 & 0.1883 & 0.1267 & 225.5414 & 0.1835 & 1.5823 & 181.3951 & 0.1258 \\
\hline 12 & 0.3091 & 476.5703 & 0.1841 & 0.8668 & 326.2205 & 0.1881 & 0.1267 & 240.3324 & 0.1822 & 1.5823 & 193.8192 & 0.1272 \\
\hline 13 & 0.3091 & 501.7973 & 0.1870 & 0.8668 & 345.4511 & 0.1880 & 0.1267 & 254.6975 & 0.1808 & 1.5823 & 206.4812 & 0.1287 \\
\hline 14 & 0.3091 & 524.0769 & 0.1902 & 0.8668 & 364.2701 & 0.1879 & 0.1267 & 268.8289 & 0.1794 & 1.5823 & 219.2939 & 0.1301 \\
\hline 15 & 0.3091 & 546.3695 & 0.1937 & 0.8668 & & & 0.1267 & & & 1.5823 & & 0.1316 \\
\hline 16 & 0.3091 & 569.8955 & 0.1972 & 0.8668 & 400.9805 & 0.1876 & 0.1267 & 296.9424 & 0.1766 & 1.5823 & 244.3629 & 0.1330 \\
\hline 17 & 0.3091 & 594.7536 & 0.2003 & 0.8668 & 418.9724 & 0.1875 & 0.1267 & 310.5833 & 0.1752 & 1.5823 & 256.2294 & 0.1344 \\
\hline 18 & 0.3091 & 620.9611 & 0.2025 & 0.8668 & 436.7805 & 0.1873 & 0.1267 & 323.7094 & 0.1739 & 1.5823 & 267.5155 & 0.1359 \\
\hline 19 & 0.3091 & 647.1204 & 0.2034 & 0.8668 & 454.4484 & 0.1872 & 0.1267 & 336.0814 & 0.1725 & 1.5823 & 278.0681 & 0.1373 \\
\hline 20 & 0.3091 & 670.2706 & 0.2030 & 0.8668 & 472.0177 & 0.1871 & 0.1267 & 347.4348 & 0.1711 & 1.5823 & 287.6962 & 0.1387 \\
\hline 21 & 0.3091 & 690.0681 & 0.2014 & 0.8668 & 489.5219 & 0.1869 & 0.1267 & 357.7775 & 0.1697 & 1.5823 & 296.3958 & 0.1402 \\
\hline 22 & 0.3091 & 706.8583 & 0.1990 & 0.8668 & 506.9924 & 0.1868 & 0.1267 & 367.1860 & 0.1683 & 1.5823 & 304.2102 & 0.1416 \\
\hline 23 & 0.3091 & 720.9831 & 0.1960 & 0.8668 & 524.4603 & 0.1866 & 0.1267 & 375.7366 & 0.1670 & 1.5823 & 311.1823 & 0.1430 \\
\hline 24 & 0.3091 & 732.2902 & 0.1926 & 0.8668 & 541.9124 & 0.1865 & 0.1267 & 383.4824 & 0.1656 & 1.5823 & 317.3075 & 0.1445 \\
\hline 25 & 0.3091 & 740.4053 & 0.1889 & 0.8668 & 559.3076 & 0.1864 & 0.1267 & 390.6086 & 0.1642 & 1.5823 & 322.5658 & 0.1459 \\
\hline 26 & 0.3091 & 747.1815 & 0.1849 & 0.8668 & 576.7470 & 0.1862 & 0.1267 & 397.7409 & 0.1628 & 1.5823 & 327.1568 & 0.1473 \\
\hline 27 & 0.3091 & 754.8518 & 0.1805 & 0.8668 & 594.3196 & 0.1861 & 0.1267 & 405.3735 & 0.1614 & 1.5823 & 331.4000 & 0.1488 \\
\hline 28 & 0.3091 & 763.4054 & 0.1758 & 0.8668 & 611.9863 & 0.1860 & 0.1267 & 413.3799 & 0.1601 & 1.5823 & 335.4719 & 0.1502 \\
\hline 29 & 0.3091 & 772.1960 & 0.1711 & 0.8668 & 629.6783 & 0.1858 & 0.1267 & 421.4867 & 0.1587 & 1.5823 & 339.4979 & 0.1516 \\
\hline 30 & 0.3091 & 780.9891 & 0.1663 & 0.8668 & 647.3706 & 0.1857 & 0.1267 & 429.5945 & 0.1573 & 1.5823 & 343.5234 & 0.1531 \\
\hline
\end{tabular}

Aortic area measured at maximum distension of the aorta on cross sectional cine gradient echo images acquired perpendicular to the aortic axis ( $\mathrm{n}=30$ ) LMS, $L=$ Lambda (skewness of the distribution), $M=M u$ (median), $S=$ Sigma (variance)

$\mathrm{z}$-score $=\left[(\mathrm{X} / \mathrm{M})^{\mathrm{L}}-1\right] /\left(\mathrm{L}^{*} \mathrm{~S}\right)$, where $\mathrm{X}$ is the measured aortic area in $\mathrm{mm}^{2}$ and $\mathrm{L}, \mathrm{M}$ and $\mathrm{S}$ are the values interpolated for the child's age; lower and upper limits correspond to a $z$-score of -2 and 2

${ }^{\text {a }}$ Age in years

${ }^{\mathrm{b}}$ Measured above the diaphragm

CMR analysis methods

Distensibility was calculated as $\left(\mathrm{A}_{\max }-\mathrm{A}_{\min }\right) / \mathrm{A}_{\min } \times\left(\mathrm{P}_{\max }\right.$ $\left.-P_{\text {min }}\right)$, where $A_{\max }$ and $A_{\text {min }}$ represent the maximal and minimal cross-sectional area of the aorta, and $\mathrm{P}_{\max }$ and $\mathrm{P}_{\text {min }}$ represent the systolic and diastolic blood pressure measured with a sphygmomanometer cuff around the right arm.

PWV was calculated as $\Delta \mathrm{x} / \Delta \mathrm{t}$, where $\Delta \mathrm{x}$ is defined as the length of the centerline between the sites of flow measurement in the ascending and descending aorta and $\Delta \mathrm{t}$ represents the time delay between the flow curve obtained in the descending aorta relative to the flow curve obtained in the ascending aorta (Fig. 12).

\section{Demographic parameters}

Aortic distensibility and PWV did not vary by gender. Aortic distensibility decreases with age and correlates with height, body weight and BSA. PWV has been shown to increase with age [92]. 
Table 45 LMS parameters to calculate z-scores for aortic cross-sectional area relative to age for girls according to reference [92]

\begin{tabular}{|c|c|c|c|c|c|c|c|c|c|c|c|c|}
\hline \multirow[t]{2}{*}{$\mathrm{Age}^{\mathrm{a}}$} & \multicolumn{3}{|c|}{ Ascending aorta } & \multicolumn{3}{|c|}{ Aortic arch } & \multicolumn{3}{|c|}{ Aortic isthmus } & \multicolumn{3}{|c|}{ Descending aorta $^{b}$} \\
\hline & $\mathbf{L}$ & M & $S$ & $\mathbf{L}$ & M & $\mathrm{S}$ & $\mathbf{L}$ & M & $S$ & $\mathbf{L}$ & M & $S$ \\
\hline$<1$ & -0.7876 & 121.1903 & 0.2152 & 2.1750 & 73.6299 & 0.2114 & 0.1033 & 60.0696 & 0.1621 & 0.9371 & 41.0795 & 0.1398 \\
\hline 1 & -0.7876 & 145.9923 & 0.2140 & 2.1750 & 92.7307 & 0.2089 & 0.1033 & 72.6142 & 0.1617 & 0.9371 & 52.4930 & 0.1398 \\
\hline 2 & -0.7876 & 170.7944 & 0.2127 & 2.1750 & 111.8315 & 0.2064 & 0.1033 & 85.1587 & 0.1613 & 9371 & 63.9065 & 0.1398 \\
\hline 3 & -0.7876 & 195.5999 & 0.2114 & 2.1750 & 130.9296 & 0.2039 & 0.1033 & 97.7032 & 0.1609 & 0.9371 & 75.3185 & 0.1398 \\
\hline 4 & -0.7876 & 220.4539 & 0.2102 & 2.1750 & 149.9904 & 0.2013 & 0.1033 & 110.2465 & 0.1605 & .9371 & 86.7100 & 0.1398 \\
\hline 5 & -0.7876 & 245.4281 & 0.2089 & 2.1750 & 168.9588 & 0.1988 & 0.1033 & 122.7870 & 0.1601 & 0.9371 & 98.0510 & 0.1398 \\
\hline 6 & -0.7876 & 270.5738 & 0.2076 & 2.1750 & 187.8089 & 0.1963 & 0.1033 & 135.3263 & 0.1597 & 0.9371 & 109.3784 & 0.1398 \\
\hline 7 & -0.7876 & 295.9027 & 0.2064 & 2.1750 & 206.5696 & 0.1938 & 0.1033 & 147.8724 & 0.1593 & 0.9371 & 120.8531 & 0.1398 \\
\hline 8 & -0.7876 & 321.3290 & 0.2051 & 2.1750 & 225.2367 & 0.1913 & 0.1033 & 160.3915 & 0.1588 & 0.9371 & 132.5201 & 0.1398 \\
\hline 9 & -0.7876 & 346.5367 & 0.2038 & 2.1750 & 243.7024 & 0.1887 & 0.1033 & 172.7395 & 0.1584 & 0.9371 & 144.0843 & 0.1398 \\
\hline 10 & -0.7876 & 371.3379 & 0.2026 & 2.1750 & 261.8643 & 0.1862 & 0.1033 & 184.8049 & 0.1580 & 0.9371 & 155.3776 & 0.1398 \\
\hline 11 & -0.7876 & 395.6874 & 0.2013 & 2.1750 & 279.6207 & 0.1837 & 0.1033 & 196.5286 & 0.1576 & 0.9371 & 166.4608 & 0.1398 \\
\hline 12 & -0.7876 & 419.5583 & 0.2000 & 2.1750 & 296.8402 & 0.1812 & 0.1033 & 207.8452 & 0.1572 & 0.9371 & 177.3057 & 0.1398 \\
\hline 13 & -0.7876 & 442.8024 & 0.1988 & 2.1750 & 313.4236 & 0.1787 & 0.1033 & 218.7232 & 0.1568 & 0.9371 & 187.8984 & 0.1398 \\
\hline 14 & -0.7876 & 465.1326 & & & 329.2852 & & 0.1033 & 229.1136 & 0.1564 & 0.9371 & 198.1163 & 0.1398 \\
\hline 15 & -0.7876 & 486.2071 & 0.1962 & 2.1750 & 344.3674 & 0.1736 & 0.1033 & 238.9630 & 0.1560 & 0.9371 & 207.7776 & 0.1398 \\
\hline 16 & -0.7876 & 505.7398 & 0.1950 & 2.1750 & 358.6387 & 0.1711 & 0.1033 & 248.2461 & 0.1556 & 0.9371 & 216.7982 & 0.1398 \\
\hline 17 & -0.7876 & 523.5836 & 0.1937 & 2.1750 & 372.0983 & 0.1686 & 0.1033 & 256.9723 & 0.1552 & 0.9371 & 225.1710 & 0.1398 \\
\hline 18 & -0.7876 & 539.7165 & 0.1924 & 2.1750 & 384.7434 & 0.1661 & 0.1033 & 265.1479 & 0.1547 & 0.9371 & 232.7857 & 0.1398 \\
\hline 19 & -0.7876 & 554.1764 & 0.1912 & 2.1750 & 396.5833 & & 0.1033 & 272.7929 & 0.1543 & 0.9371 & 239.5830 & 0.1398 \\
\hline 20 & -0.7876 & 567.1207 & 0.1899 & 2.1750 & 407.6567 & 0.1610 & 0.1033 & 279.9469 & 0.1539 & 0.9371 & 245.6109 & 0.1398 \\
\hline 21 & -0.7876 & 578.7817 & 0.1886 & 2.1750 & 418.0442 & 0.1585 & 0.1033 & 286.6730 & 0.1535 & 0.9371 & 251.0496 & 0.1398 \\
\hline 22 & -0.7876 & 589.4770 & 0.1873 & 2.1750 & 427.8971 & 0.1560 & 0.1033 & 293.0630 & 0.1531 & 0.9371 & 256.1671 & 0.1398 \\
\hline 23 & -0.7876 & 599.5300 & 0.1861 & 2.1750 & 437.3887 & 0.1534 & 0.1033 & 299.2101 & 0.1527 & 0.9371 & 261.1406 & 0.1398 \\
\hline 24 & -0.7876 & 609.3164 & 0.1848 & 2.1750 & 446.7229 & 0.1509 & 0.1033 & 305.2232 & 0.1523 & 0.9371 & 266.1367 & 0.1398 \\
\hline 25 & -0.7876 & 619.1593 & 0.1835 & 2.1750 & 456.0570 & 0.1484 & 0.1033 & 311.2003 & 0.1518 & 0.9371 & 271.2515 & 0.1398 \\
\hline 26 & -0.7876 & 629.1747 & 0.1822 & 2.1750 & 465.4360 & 0.1459 & 0.1033 & 317.2057 & 0.1514 & 0.9371 & 276.5640 & 0.1398 \\
\hline 27 & -0.7876 & 639.3019 & 0.1810 & 2.1750 & 474.8382 & 0.1433 & 0.1033 & 323.2314 & 0.1510 & 0.9371 & 281.9813 & 0.1398 \\
\hline 28 & -0.7876 & 649.4860 & 0.1797 & 2.1750 & 484.2530 & 0.1408 & 0.1033 & 329.2650 & 0.1506 & 0.9371 & 287.4341 & 0.1398 \\
\hline 29 & -0.7876 & 659.6776 & 0.1784 & 2.1750 & 493.6694 & 0.1383 & 0.1033 & 335.2995 & 0.1502 & 0.9371 & 292.8916 & 0.1398 \\
\hline 30 & -0.7876 & 669.8691 & 0.1772 & 2.1750 & 503.0858 & 0.1358 & 0.1033 & 341.3341 & 0.1498 & 0.9371 & 298.3491 & 0.1398 \\
\hline
\end{tabular}

Aortic area measured at maximum distension of the aorta on cross sectional cine gradient echo images acquired perpendicular to the aortic axis ( $\mathrm{n}=41$ )

LMS, $L=$ Lambda (skewness of the distribution), $M=M u$ (median), $S=$ Sigma (variance)

$\mathrm{z}$-score $=\left[(\mathrm{X} / \mathrm{M})^{\mathrm{L}}-1\right] /\left(\mathrm{L}^{*} \mathrm{~S}\right)$, where $\mathrm{X}$ is the measured aortic area in $\mathrm{mm}^{2}$ and $\mathrm{L}, \mathrm{M}$ and $\mathrm{S}$ are the values interpolated for the child's age; lower and upper limits correspond to a $\mathrm{z}$-score of -2 and 2

${ }^{\text {a }}$ Age in years

${ }^{\mathrm{b}}$ Measured above the diaphragm

\section{Studies included in this review}

There is a single publication only of a systematic evaluation of normal aortic distensibility and PWV in children (Table 52). In this review we present LMS parameters to calculate z-scores for distensibility of the ascending aorta and PWV based on reference [92] (Tables 53, 54). In the original publication LMS parameters for distensibility at 3 other levels of the thoracic aorta (aortic arch, aortic isthmus and distal descending aorta) are presented in addition [92].

\section{Normal dimensions and distension of the pulmonary arteries in adults CMR acquisition parameters}

In the study by Burman et al. listed in this review [99] dimensions of the pulmonary arteries were measured on bSSFP images (Table 55). Burman et al. acquired cross sectional images of the main and the right and left pulmonary artery based on an oblique sagittal image of the RV outflow tract and pulmonary trunk, respectively (for the main pulmonary artery) and an axial image acquired 
Table 46 Normal aortic diameters in children measured on a contrast enhanced 3D-CMRA according to reference [91]

\begin{tabular}{lll}
\hline Site & Predicted diameter $(\mathbf{m m})$ & $\begin{array}{l}\text { SD of } \\
\text { residuals } \\
(\mathbf{m m})\end{array}$ \\
\hline Aortic sinus & $0.57+19.37^{*} \mathrm{BSA}^{0.5}$ & 2.38 \\
Sinotubular junction & $-0.03+16.91^{*} \mathrm{BSA}^{0.5}$ & 1.92 \\
Ascending aorta & $-1.33+18.6^{*} \mathrm{BSA}^{0.5}$ & 1.99 \\
Proximal to the origin of the & $-3.38+20.07^{*} \mathrm{BSA}^{0.5}$ & 1.69 \\
brachiocephalic artery & $-3.52+18.66^{*} \mathrm{BSA}^{0.5}$ & 1.63 \\
First transverse segment & $-2.63+16.5^{*} \mathrm{BSA}^{0.5}$ & 1.31 \\
$\begin{array}{l}\text { Second transverse segment } \\
\text { Isthmic región }\end{array}$ & $-3.37+16.52^{*} \mathrm{BSA}^{0.5}$ & 1.46 \\
$\begin{array}{l}\text { Descending aorta } \\
\text { Thoracoabdominal aorta at }\end{array}$ & $1.27+9.89^{*} \mathrm{BSA}^{0.5}$ & 1.64 \\
the level of the diaphragm & & 1.34 \\
\hline
\end{tabular}

Shortest diameter measured on cross-sectional reformatted images $(n=53)$. Sites of measurement are shown in Fig. 9

$\mathrm{z}$-score $=$ (measured diameter - predicted diameter $) / S D$ of residuals; lower and upper limits correspond to a $\mathrm{z}$-score of -2 and 2

$B S A$ body surface area, $S D$ standard deviation

Table 47 Normal aortic area on phase contrast cine images according to reference [93]

\begin{tabular}{lc}
\hline Site & Predicted area $\left(\mathbf{c m}^{\mathbf{2}}\right)$ \\
\hline Ascending aorta & $-0.0386+2.913^{*} \mathrm{BSA}$
\end{tabular}

Cross sectional area calculated based on measurement of the maximal external aortic diameter perpendicular to the vessel and perpendicular to the maximal diameter in systole 1 to $2 \mathrm{~cm}$ distal to the sinotubular junction on the magnitude image of a phase contrast cine sequence $(n=105)$

$B S A$ body surface area

at the level of the bifurcation of the main pulmonary artery (for the left and right pulmonary artery) (Fig. 13). With three-dimensional acquisition, reconstruction of the imaging plane can be performed after image acquisition using multiplanar reformation.

Other sequences could also be used to obtain dimensions of the pulmonary arteries such as a three-dimensional contrast enhanced CMRA with contrast timing optimized to enhance the pulmonary arteries. Non-contrast techniques include respiratory and ECG-gated 3D bSSFP sequence and cine phase contrast imaging. However, similar to the aorta, measurements of the pulmonary artery are expected to vary by the sequence type and might not be comparable [82]. In contrast to static sequences, acquisition of dynamic sequences, e.g. cine bSSFP, enable measurements at systole and diastole and calculation of distension.

\section{CMR analysis methods}

Luminal areas and diameters of the pulmonary arteries were measured on cross sectional images of the respective
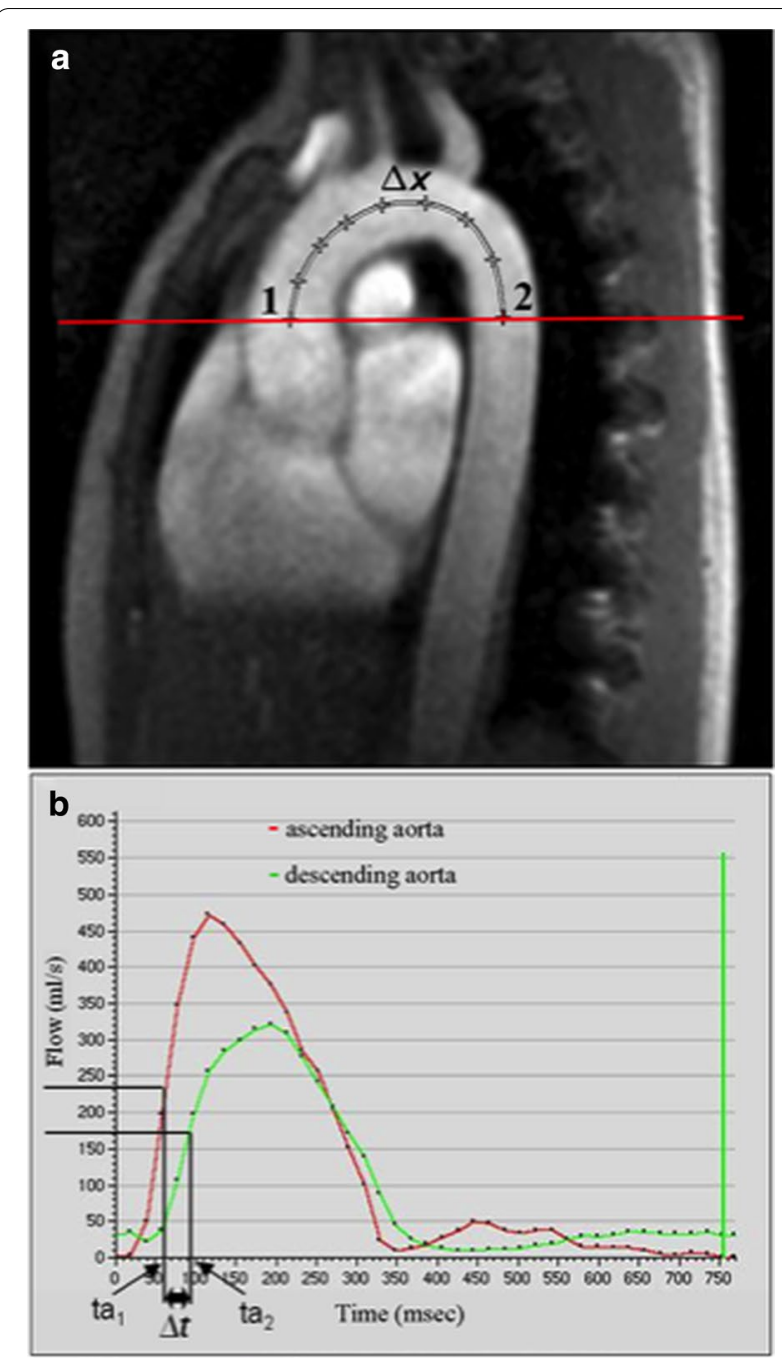

Fig. 12 Measurement of pulse wave velocity according to reference [92]. $\Delta x$ : length of the centerline between the sites of flow measurement in the ascending and descending aorta; $\Delta \mathrm{t}$ : time delay between the flow curves obtained in the descending aorta relative to the flow curve obtained in the ascending aorta calculated between the midpoint of the systolic up slope tails on the flow versus time curves of the ascending aorta (ta1) and the descending aorta (ta2)

vessel at minimal diastolic and minimal systolic expansion. Since the cross section of the vessel is usually not perfectly circular, data presented in Table 56 shows the mean diameter of two diameters that were acquired per vessel and phase calculated from the greatest diameter and the lesser diameter orthogonal to the greater diameter. Percent systolic distension was calculated as [(maximum area - minimum area) $* 100 /$ minimum area].

\section{Demographic parameters}

Area and mean diameters of the pulmonary arteries are greater in men compared to women and greater in 
Table 48 References, normal aortic pulse wave velocity (PWV) and distensibility

\begin{tabular}{lllc}
\hline First author, year & CMR technique & n, male:female & Age range (years) \\
\hline Kim, 2013 [98] & $\begin{array}{l}\text { 1.5 T, phase contrast CMR to calculate PWV for 3 distances of the aorta; transit time } \\
\text { calculated from the midpoint of the systolic up-slope on the flow versus time curve; cross } \\
\text { sectional cine bSSFP at 4 levels of the aorta to calculate distensibility }\end{array}$ & $61: 63$ & $20-79$ \\
Eikendal, 2016 [84] & $\begin{array}{l}\text { 3T, phase contrast CMR to calculate PWV for 2 distances of the aorta, time delay calculated } \\
\text { from velocity-time curves }\end{array}$ & $57: 61$ & $25-35$
\end{tabular}

$n$ number of study subjects, bSSFP balanced steady-state free precession, $P W V$ pulse wave velocity

Table 49 Normal values of regional aortic distensibility for men and women according to [98]

\begin{tabular}{|c|c|c|c|}
\hline Level & Age (years) & $\begin{array}{l}\text { Men }(n=61) \\
\text { Mean } \pm S D \\
\left(10^{-3} \mathrm{~mm} /\right. \\
\mathrm{Hg})\end{array}$ & $\begin{array}{l}\text { Women } \\
(\mathrm{n}=63) \\
\text { Mean } \pm \mathrm{SD} \\
\left(10^{-3} \mathrm{~mm} /\right. \\
\mathrm{Hg})\end{array}$ \\
\hline \multirow[t]{5}{*}{ Ascending aorta ${ }^{a}$} & $20-29$ & $5.6 \pm 1.5$ & $7.9 \pm 3.4$ \\
\hline & $30-39$ & $3.6 \pm 1.4$ & $6.5 \pm 3.0$ \\
\hline & $40-49$ & $3.5 \pm 1.5$ & $5.3 \pm 1.2$ \\
\hline & $50-59$ & $3.2 \pm 1.6$ & $3.6 \pm 1.1$ \\
\hline & $60-69$ & $2.1 \pm 1.3$ & $2.7 \pm 1.0$ \\
\hline \multirow[t]{5}{*}{ Proximal descending aorta ${ }^{a}$} & $20-29$ & $4.2 \pm 0.9$ & $6.0 \pm 1.4$ \\
\hline & $30-39$ & $3.8 \pm 1.3$ & $5.5 \pm 1.9$ \\
\hline & $40-49$ & $3.3 \pm 0.6$ & $4.2 \pm 1.2$ \\
\hline & $50-59$ & $2.9 \pm 1.1$ & $3.7 \pm 1.3$ \\
\hline & $60-69$ & $2.3 \pm 0.9$ & $3.1 \pm 0.9$ \\
\hline
\end{tabular}

$n$ number of study subjects

${ }^{\text {a }}$ Measurements obtained at the level of the bifurcation of the pulmonary artery

Table 50 Normal values for aortic pulse wave velocity according to [98]

\begin{tabular}{lll}
\hline Age (years) & $\mathbf{n}$ & $\begin{array}{l}\text { Median } \\
\text { (5th-95th } \\
\text { percentile) }(\mathbf{m} / \mathbf{s})\end{array}$ \\
\hline $20-29$ & 26 & $3.7(3.4-4.0)$ \\
$30-39$ & 28 & $3.8(3.5-6.0)$ \\
$40-49$ & 24 & $4.3(3.7-5.0)$ \\
$50-59$ & 25 & $5.6(5.4-7.2)$ \\
$60-69$ & 21 & $9.0(7.4-12.4)$ \\
\hline
\end{tabular}

Regional pulse wave velocity from the ascending to the upper descending thoracic aorta

$n$ number of study subjects

systole compared to diastole. Some measurements of the area and the mean diameter of the pulmonary arteries slightly increase with BSA and age, while systolic distension decrease with age. For a detailed description of the relationship of the area, mean diameters and systolic distension of the pulmonary arteries with age and BSA please see [99].
Table 51 Normal values for aortic pulse wave velocity in young men and women (25-35 years) according to [84]

\begin{tabular}{ll}
\hline $\begin{array}{l}\text { Men }(\mathbf{n}=\mathbf{5 7}) \\
\text { Median (10th-90th percentile) } \\
(\mathbf{m} / \mathbf{s})\end{array}$ & $\begin{array}{l}\text { Women }(\mathbf{n}=61) \\
\text { Median (10th-90th percentile) } \\
(\mathbf{m} / \mathbf{s})\end{array}$ \\
\hline $4.6(3.9-5.6)$ & $4.5(3.6-6.0)$
\end{tabular}

Regional pulse wave velocity from the ascending to the upper descending thoracic aorta

$n$ number of study subjects

\section{Studies included in this review}

One publication of reference ranges of the area, diameters and distension of the pulmonary arteries in adults was found using a current CMR technique, sufficient sample size ( $>40$ subjects per gender) and a clear description of image acquisition and measurements [99]. In the original publication, reference ranges were presented for age deciles for subjects between 20 and 79 years with 10 subjects per decile and gender. However, since the differences between age deciles were small and might not be clinically relevant and for sample size considerations, in the current review only values of the entire cohort separated by gender are presented.

\section{Normal dimensions of the pulmonary arteries in children CMR acquisition parameters}

In analogy to dimensions of the pulmonary arteries in adults, different sequences might be used to obtain measurements. In the studies listed below, a contrast enhanced three-dimensional CMRA and a cross sectional through-plane free-breathing phase contrast sequence were acquired to obtain the measurements $[93,100]$.

\section{CMR analysis methods}

Knobel et al. obtained measurements of the pulmonary arteries on reconstructed maximum intensity projection (MIP) images (slice thickness is not mentioned) perpendicular to the respective vessel (Fig. 14) [100]. The 
Table 52 References, normal distensibility and pulse wave velocity (PWV) in children

\begin{tabular}{llll} 
First author, year & CMR technique & n, male:female & Age range (years) \\
\hline Voges, 2012 [92] & $\begin{array}{l}\text { 3T; cross sectional cine GRE at 4 levels of the thoracic aorta to calculate distensibility; phase } \\
\text { contrast CMR to calculate PWV for the distance between the sinotubular junction and the } \\
\begin{array}{l}\text { proximal descending aorta, transit time calculated from the midpoint of the systolic up- } \\
\text { slope on the flow versus time curve }\end{array}\end{array}$ & $30: 41$ & $2-28$
\end{tabular}

$n$ number of study subjects, GRE gradient echo, $P W V$ pulse wave velocity

Table 53 LMS parameters to calculate z-scores for distensibility of the ascending aorta relative to age in children according to reference [92]

\begin{tabular}{|c|c|c|c|c|c|c|}
\hline \multirow[t]{2}{*}{ Age (years) } & \multicolumn{3}{|c|}{ Male $(n=30)$} & \multicolumn{3}{|c|}{ Female $(n=41)$} \\
\hline & $\mathrm{L}$ & $M$ & $S$ & $\mathrm{~L}$ & $M$ & $S$ \\
\hline$<1$ & -0.1879 & 12.3602 & 0.3680 & -0.0721 & 12.7303 & 0.2388 \\
\hline 1 & -0.1879 & 11.9220 & 0.3680 & -0.0721 & 12.5028 & 0.2396 \\
\hline 2 & -0.1879 & 11.4838 & 0.3680 & -0.0721 & 12.2753 & 0.2403 \\
\hline 3 & -0.1879 & 11.0456 & 0.3680 & -0.0721 & 12.0477 & 0.2411 \\
\hline 4 & -0.1879 & 10.6075 & 0.3680 & -0.0721 & 11.8176 & 0.2419 \\
\hline 5 & -0.1879 & 10.1700 & 0.3680 & -0.0721 & 11.5817 & 0.2427 \\
\hline 6 & -0.1879 & 9.7343 & 0.3680 & -0.0721 & 11.3421 & 0.2435 \\
\hline 7 & -0.1879 & 9.2990 & 0.3680 & -0.0721 & 11.1121 & 0.2443 \\
\hline 8 & -0.1879 & 8.8602 & 0.3680 & -0.0721 & 10.9051 & 0.2451 \\
\hline 9 & -0.1879 & 8.4151 & 0.3680 & -0.0721 & 10.7290 & 0.2459 \\
\hline 10 & -0.1879 & 7.9776 & 0.3680 & -0.0721 & 10.5679 & 0.2467 \\
\hline 11 & -0.1879 & 7.5683 & 0.3680 & -0.0721 & 10.3851 & 0.2474 \\
\hline 12 & -0.1879 & 7.2051 & 0.3680 & -0.0721 & 10.1582 & 0.2482 \\
\hline 13 & -0.1879 & 6.9030 & 0.3680 & -0.0721 & 9.8884 & 0.2490 \\
\hline 14 & -0.1879 & 6.6697 & 0.3680 & -0.0721 & 9.5911 & 0.2498 \\
\hline 15 & -0.1879 & 6.5089 & 0.3680 & -0.0721 & 9.2905 & 0.2506 \\
\hline 16 & -0.1879 & 6.4138 & 0.3680 & -0.0721 & 9.0033 & 0.2514 \\
\hline 17 & -0.1879 & 6.3729 & 0.3680 & -0.0721 & 8.7345 & 0.2522 \\
\hline 18 & -0.1879 & 6.3745 & 0.3680 & -0.0721 & 8.4850 & 0.2529 \\
\hline 19 & -0.1879 & 6.4062 & 0.3680 & -0.0721 & 8.2574 & 0.2537 \\
\hline 20 & -0.1879 & 6.4551 & 0.3680 & -0.0721 & 8.0546 & 0.2545 \\
\hline 21 & -0.1879 & 6.5111 & 0.3680 & -0.0721 & 7.8749 & 0.2553 \\
\hline 22 & -0.1879 & 6.5646 & 0.3680 & -0.0721 & 7.7106 & 0.2561 \\
\hline 23 & -0.1879 & 6.6062 & 0.3680 & -0.0721 & 7.5479 & 0.2569 \\
\hline 24 & -0.1879 & 6.6277 & 0.3680 & -0.0721 & 7.3842 & 0.2577 \\
\hline 25 & -0.1879 & 6.6242 & 0.3680 & -0.0721 & 7.2113 & 0.2584 \\
\hline 26 & -0.1879 & 6.5975 & 0.3680 & -0.0721 & 7.0343 & 0.2592 \\
\hline 27 & -0.1879 & 6.5577 & 0.3680 & -0.0721 & 6.8647 & 0.2600 \\
\hline 28 & -0.1879 & 6.5116 & 0.3680 & -0.0721 & 6.6951 & 0.2608 \\
\hline 29 & -0.1879 & 6.4643 & 0.3680 & -0.0721 & 6.5250 & 0.2616 \\
\hline 30 & -0.1879 & 6.4170 & 0.3680 & -0.0721 & 6.3550 & 0.2624 \\
\hline
\end{tabular}

Distensibility was calculated based on measurements of the aortic area at systole and diastole on cross sectional cine gradient echo images obtained perpendicular to the axis of the ascending thoracic aorta.

LMS, $\mathrm{L}=\mathrm{Lambda}$ (skewness of the distribution), $\mathrm{M}=\mathrm{Mu}$ (median), $\mathrm{S}=$ Sigma (variance).

$z$-score $=\left[(X / M)^{L}-1\right] /\left(L^{*} S\right)$, where $X$ is the measured aortic distensibility in $10^{-3} \mathrm{~mm} \mathrm{Hg}^{-1}$ and $\mathrm{L}, \mathrm{M}$ and $\mathrm{S}$ are the values interpolated for the child's age; lower and upper limits correspond to a z-score of -2 and 2.

$n$ number of study subjects. 
Table 54 LMS parameters to calculate z-scores for pulse wave velocity (PWV) relative to age in children according to reference [92]

\begin{tabular}{|c|c|c|c|c|c|c|}
\hline \multirow[t]{2}{*}{ Age (years) } & \multicolumn{3}{|c|}{ Male $(n=30)$} & \multicolumn{3}{|c|}{ Female $(n=41)$} \\
\hline & $\mathrm{L}$ & $M$ & $\mathrm{~S}$ & $\mathrm{~L}$ & $M$ & $S$ \\
\hline$<1$ & 1.4844 & 3.4147 & 0.2122 & -1.5196 & 2.7808 & 0.1468 \\
\hline 1 & 1.4844 & 3.4367 & 0.2122 & -1.5196 & 2.8144 & 0.1469 \\
\hline 2 & 1.4844 & 3.4587 & 0.2122 & -1.5196 & 2.8481 & 0.1469 \\
\hline 3 & 1.4844 & 3.4808 & 0.2122 & -1.5196 & 2.8817 & 0.1469 \\
\hline 4 & 1.4844 & 3.5028 & 0.2122 & -1.5196 & 2.9154 & 0.1470 \\
\hline 5 & 1.4844 & 3.5248 & 0.2122 & -1.5196 & 2.9490 & 0.1470 \\
\hline 6 & 1.4844 & 3.5469 & 0.2122 & -1.5196 & 2.9827 & 0.1470 \\
\hline 7 & 1.4844 & 3.5689 & 0.2122 & -1.5196 & 3.0163 & 0.1470 \\
\hline 8 & 1.4844 & 3.5909 & 0.2122 & -1.5196 & 3.0499 & 0.1471 \\
\hline 9 & 1.4844 & 3.6129 & 0.2122 & -1.5196 & 3.0836 & 0.1471 \\
\hline 10 & 1.4844 & 3.6350 & 0.2122 & -1.5196 & 3.1172 & 0.1471 \\
\hline 11 & 1.4844 & 3.6570 & 0.2122 & -1.5196 & 3.1509 & 0.1471 \\
\hline 12 & 1.4844 & 3.6790 & 0.2122 & -1.5196 & 3.1845 & 0.1472 \\
\hline 13 & 1.4844 & 3.7011 & 0.2122 & -1.5196 & 3.2182 & 0.1472 \\
\hline 14 & 1.4844 & 3.7231 & 0.2122 & -1.5196 & 3.2518 & 0.1472 \\
\hline 15 & 1.4844 & 3.7451 & 0.2122 & -1.5196 & 3.2855 & 0.1473 \\
\hline 16 & 1.4844 & 3.7672 & 0.2122 & -1.5196 & 3.3192 & 0.1473 \\
\hline 17 & 1.4844 & 3.7892 & 0.2122 & -1.5196 & 3.3528 & 0.1473 \\
\hline 18 & 1.4844 & 3.8112 & 0.2122 & -1.5196 & 3.3865 & 0.1473 \\
\hline 19 & 1.4844 & 3.8333 & 0.2122 & -1.5196 & 3.4201 & 0.1474 \\
\hline 20 & 1.4844 & 3.8553 & 0.2122 & -1.5196 & 3.4538 & 0.1474 \\
\hline 21 & 1.4844 & 3.8773 & 0.2122 & -1.5196 & 3.4875 & 0.1474 \\
\hline 22 & 1.4844 & 3.8994 & 0.2122 & -1.5196 & 3.5211 & 0.1475 \\
\hline 23 & 1.4844 & 3.9214 & 0.2122 & -1.5196 & 3.5548 & 0.1475 \\
\hline 24 & 1.4844 & 3.9434 & 0.2122 & -1.5196 & 3.5885 & 0.1475 \\
\hline 25 & 1.4844 & 3.9655 & 0.2122 & -1.5196 & 3.6221 & 0.1476 \\
\hline 26 & 1.4844 & 3.9875 & 0.2122 & -1.5196 & 3.6558 & 0.1476 \\
\hline 27 & 1.4844 & 4.0096 & 0.2122 & -1.5196 & 3.6895 & 0.1476 \\
\hline 28 & 1.4844 & 4.0316 & 0.2122 & -1.5196 & 3.7231 & 0.1476 \\
\hline 29 & 1.4844 & 4.0536 & 0.2122 & -1.5196 & 3.7568 & 0.1477 \\
\hline 30 & 1.4844 & 4.0757 & 0.2122 & -1.5196 & 3.7905 & 0.1477 \\
\hline
\end{tabular}

Pulse wave velocity calculated by phase contrast CMR for the distance between the sinotubular junction and the proximal descending aorta. Transit time calculated from the midpoint of the systolic up-slope on the flow versus time curve (Fig. 12).

LMS, $\mathrm{L}=$ Lambda (skewness of the distribution), $M=M u$ (median), $S=$ Sigma (variance)

$z$-score $=\left[(X / M)^{L}-1\right] /\left(L^{*} S\right)$, where $X$ is the measured pulse wave velocity in $m / s$ and $L, M$ and $S$ are the values interpolated for the child's age; lower and upper limits correspond to a $z$-score of -2 and 2 .

$n$ number of study subjects

Table 55 Reference, normal dimensions and distension of the pulmonary arteries in adults

\begin{tabular}{llll}
\hline First author, year & CMR technique & n, male:female & Age range (years) \\
\hline Burman, 2016 [99] & 1.5 T, cross sectional bSSFP, luminal area and mean diameters & $60: 60$ & $20-79$
\end{tabular}

$n$ number of study subjects, bSSFP balanced steady-state free precession

diameter of the main pulmonary artery was obtained on an axial and a reformatted sagittal oblique view, the diameters of the proximal and distal right and left pulmonary artery were measured on an axial and reformatted right and left anterior oblique (coronal oblique) views. 

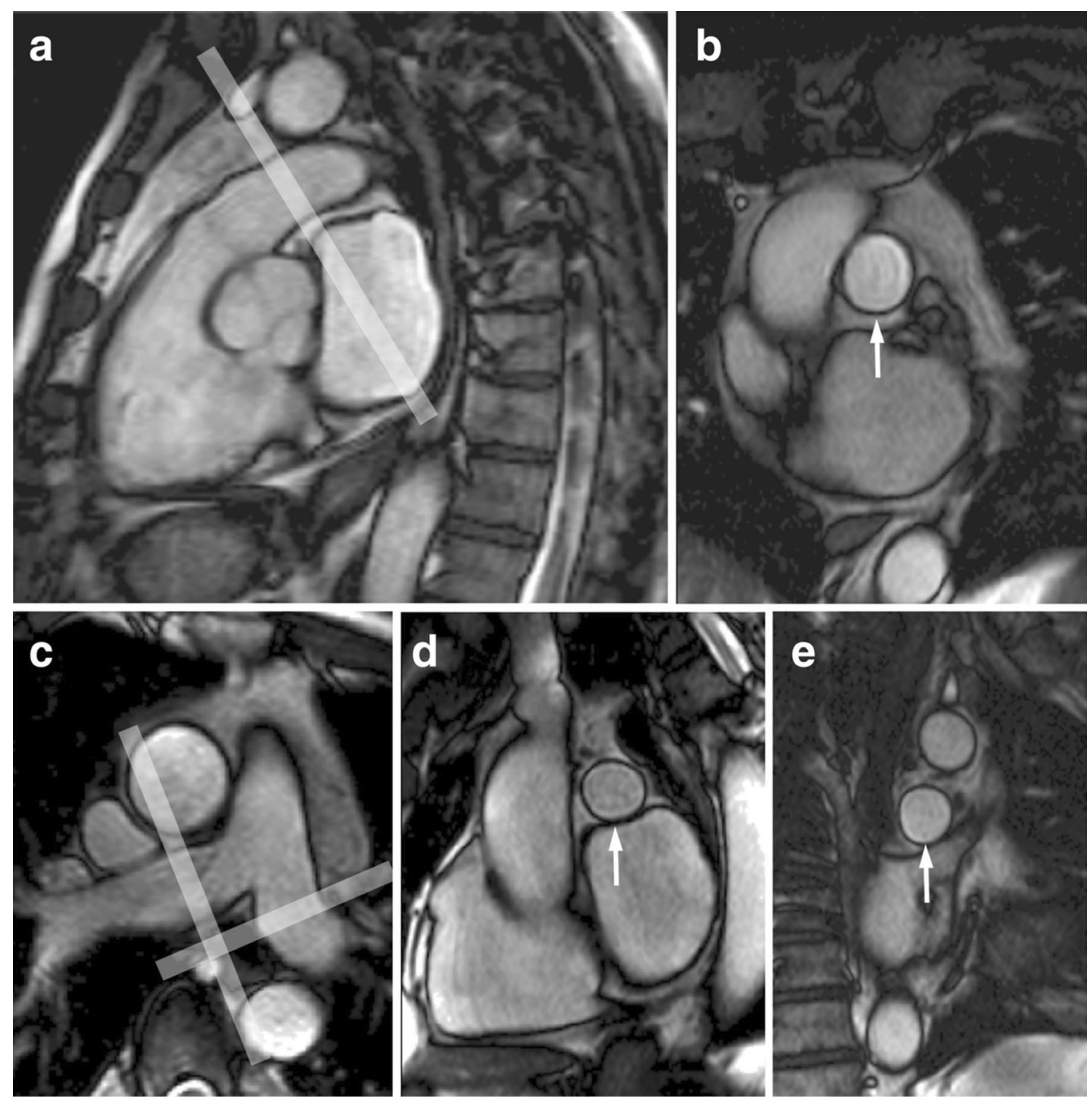

Fig. 13 Measurement of the dimensions of the pulmonary arteries on bSSFP images according to [99]. Oblique sagittal image of the main pulmonary artery (a). The pale band in $\mathbf{a}$ shows the acquisition plane of the cross sectional image of the main pulmonary artery in $\mathbf{b}$. Right and left pulmonary arteries on the scout image (c) with band indicating the location of cine acquisitions transecting the right (d) and left (e) pulmonary artery

In the study by Kutty et al. the maximal external diameter of the main pulmonary artery $\left(d_{1}\right)$ was measured on the cross sectional magnitude image of the PC sequence in systole and also the diameter $\left(d_{2}\right)$ perpendicular to $d_{1}$ [93]. After derivation of the radii $\left(r_{1}\right.$ and $\left.r_{2}\right)$, the area was calculated as $\pi r_{1} r_{2}$.

\section{Demographic parameters}

In both studies a relationship between pulmonary artery diameter and BSA was described [93, 100]. Kutty et al. could not find a significant gender difference of the size of the main pulmonary artery.

\section{Studies included in this review}

Two studies were identified presenting normal values of the size of the pulmonary arteries in children [93, 100] (Table 57). Knobel et al. included 69 children ranging from 2 to 20 years with a previous history of malignancy that were assessed for potential port-a-cath related complications but normal cardiovascular anatomy and 
Table 56 Normal dimensions and distension of the pulmonary arteries in adults according to [99]

\begin{tabular}{|c|c|c|c|c|c|c|c|}
\hline \multirow[t]{2}{*}{ Vessel } & \multirow[t]{2}{*}{ Parameter } & \multicolumn{3}{|c|}{ Men $(n=60)$} & \multicolumn{3}{|c|}{ Women $(n=60)$} \\
\hline & & Mean & SD & $\overline{L L-U L^{a}}$ & Mean & SD & $\mathrm{LL}^{\mathrm{U}} \mathrm{L}^{\mathrm{a}}$ \\
\hline \multirow[t]{5}{*}{ MPA } & Systolic diameter (mm) & 27.4 & 2.6 & $21-33$ & 25.3 & 2.6 & $19-31$ \\
\hline & Diastolic diameter (mm) & 22.9 & 2.4 & $19-27$ & 21.2 & 2.1 & $17-25$ \\
\hline & Systolic area $\left(\mathrm{cm}^{2}\right)$ & 5.9 & 1.1 & $3.7-8.1$ & 5.0 & 1.0 & $3.0-7.0$ \\
\hline & Diastolic area $\left(\mathrm{cm}^{2}\right)$ & 4.2 & 0.8 & $2.6-5.8$ & 3.6 & 0.7 & $2.2-5.0$ \\
\hline & Distension (\%) & 42.7 & 17.2 & $9-77$ & 41.8 & 15.7 & $10-74$ \\
\hline \multirow[t]{5}{*}{ RPA } & Systolic diameter (mm) & 20.2 & 2.9 & $14-26$ & 17.8 & 2.4 & $14-22$ \\
\hline & Diastolic diameter (mm) & 16.6 & 2.8 & $11-23$ & 14.7 & 2.2 & $11-19$ \\
\hline & Systolic area $\left(\mathrm{cm}^{2}\right)$ & 3.3 & 1.0 & $1.3-5.3$ & 2.6 & 0.7 & $1.2-4.0$ \\
\hline & Diastolic area $\left(\mathrm{cm}^{2}\right)$ & 2.2 & 0.8 & $0.6-3.8$ & 1.8 & 0.6 & $0.6-3.0$ \\
\hline & Distension (\%) & 50.6 & 16.9 & $17-85$ & 48.2 & 14.5 & $18-78$ \\
\hline \multirow[t]{5}{*}{ LPA } & Systolic diameter (mm) & 20.1 & 2.4 & $16-24$ & 18.4 & 2.1 & $14-22$ \\
\hline & Diastolic diameter $(\mathrm{mm})$ & 17.3 & 2.5 & $11-23$ & 15.9 & 2.0 & $12-20$ \\
\hline & Systolic area $\left(\mathrm{cm}^{2}\right)$ & 3.3 & 0.8 & $1.7-4.9$ & 2.8 & 0.6 & $1.6-4.0$ \\
\hline & Diastolic area $\left(\mathrm{cm}^{2}\right)$ & 2.4 & 0.7 & $1.0-3.8$ & 2.1 & 0.5 & $1.1-3.1$ \\
\hline & Distension (\%) & 35.6 & 10.1 & $16-56$ & 35.2 & 10.3 & $15-55$ \\
\hline
\end{tabular}

$n$ number of study subjects, SD standard deviation, $L L$ lower limit, UL upper limit, MPA main pulmonary artery, RPA right pulmonary artery, LPA left pulmonary artery

${ }^{\text {a }}$ Calculated as mean $\pm 2 * \mathrm{SD}$

no evidence of cardiovascular disease [100] (Table 58). In the study by Kutty et al. 105 normal healthy subjects between 4 and 20 years were included (data presented here; Table 59) and also subjects with repaired tetralogy of Fallot (not presented in this review) [93].

Due to the differences in sequence type, measurement technique and data presentation the normal values of the two studies are presented separately.

\section{Normal values of myocardial T1 relaxation time and the extracellular volume (ECV) CMR acquisition parameters}

The field of myocardial T1 mapping has matured significantly with several studies reporting $\mathrm{T} 1$ relaxation times for normal cohorts [101]. An Expert Consensus document on parametric mapping has been published providing recommendations for the practical clinical application of T1, T2, T2*, and ECV mapping [102]. Most of the published myocardial T1 values have been acquired using variants of the Modified Look-Locker Inversion Recovery (MOLLI) technique [103] including the shortened-MOLLI (ShMOLLI) [104] method. Saturation recovery based techniques such as saturation recovery single-shot acquisition (SASHA) are alternative techniques but have less clinical evidence to date [105]. Images are typically acquired in diastole to limit cardiac motion and respiratory motion correction.

Native T1 maps are acquired without a contrast agent. Post contrast T1 maps allow assessment of gadolinium contrast distribution, as these agents shorten the $\mathrm{T} 1$ relaxation time of water. T1 maps acquired 10-30 min following injection of an extra-cellular non-protein binding gadolinium contrast agent can be used to quantify the extracellular volume fraction (ECV) [102]. Post contrast T1 values have been performed following a bolus or primed infusion (Equilibrium-EQCMR) with good agreement of ECV values up to $40 \%$ [106]. While the hematocrit can be approximated from the T1 of the blood in the LV cavity ("synthetic T1"), assessment of hematocrit by blood sampling as close as possible in time to the CMR (less than $24 \mathrm{~h}$ ) is preferred due to normal daily variation of hematocrit [102].

\section{Factors affecting $\mathrm{T} 1$ relaxation time and ECV}

There are a number of CMR acquisition factors that can affect the measurement of normal T1 and ECV values. Field strength has a significant effect on T1 values; with $3 \mathrm{~T}$ scans producing $28 \%$ higher native $\mathrm{T} 1$ and $14 \%$ higher post contrast $\mathrm{T} 1$ values when compared with $1.5 \mathrm{~T}$ [107]. Post contrast $\mathrm{T} 1$ is also affected by the dose and relaxivity of the contrast agent used, contrast clearance, and the time between injection and measurement [107-109]. There is also greater heterogeneity for a T1 native normal range at $3 \mathrm{~T}[107,110,111]$. Further, it has been shown that $\mathrm{T} 1$ varies by cardiac phase (diastole versus systole) and region of measurement (septal versus non-septal) [107]. ECV values are relatively unaffected by field strength ( $3 \mathrm{~T}$ versus $1.5 \mathrm{~T}$ ). Both native T1 and ECV values have been shown to be less reliable in the inferolateral wall likely secondary to off-resonance effects $[107$, 112]. 

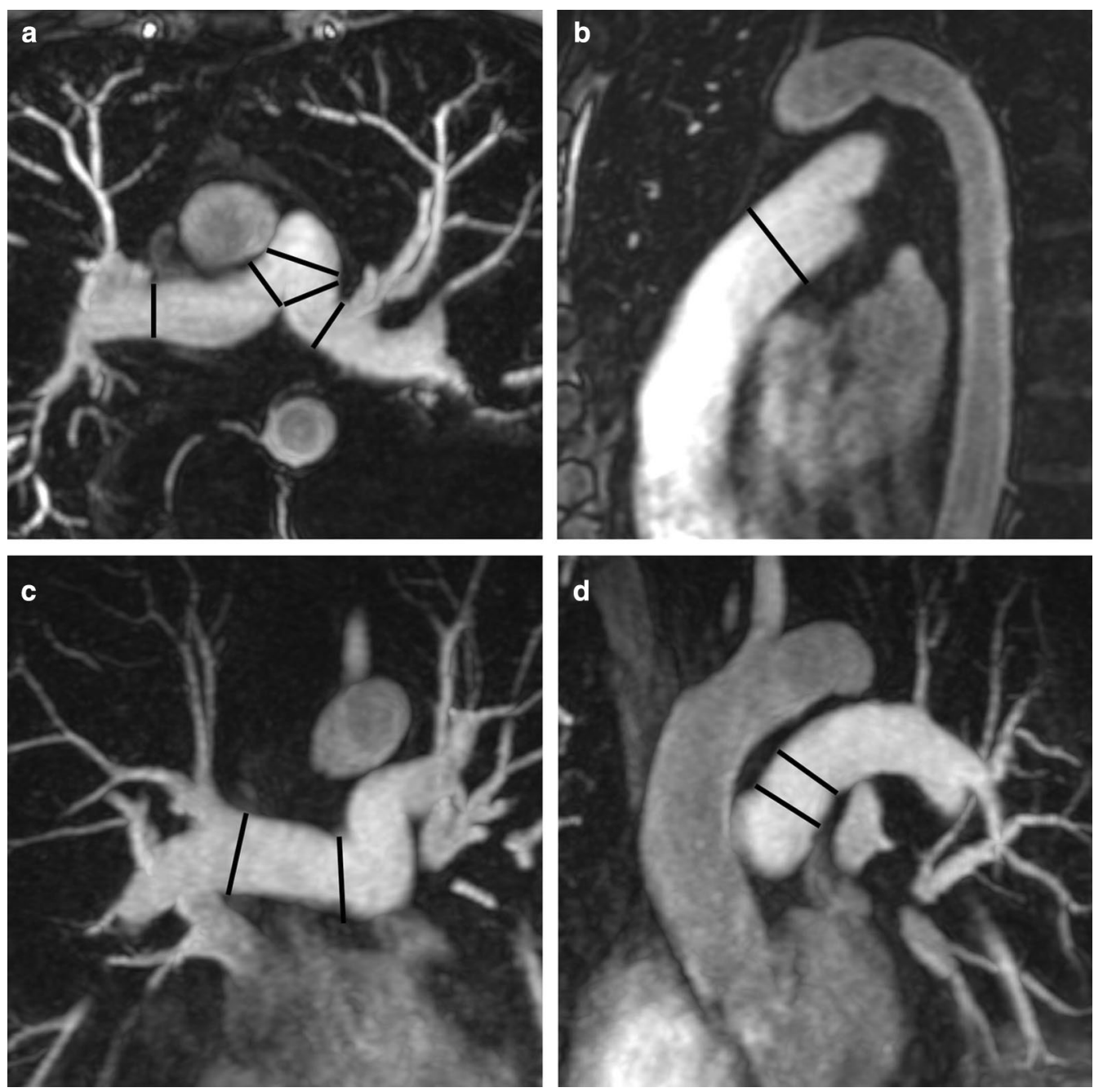

Fig. 14 Measurement of the diameters of the pulmonary arteries according to reference [100]. Diameters were measured perpendicular to the vessel on maximum intensity projection images. The diameters of the main pulmonary artery were obtained on an axial (a) and sagittal oblique (b) view and the diameters of the proximal and distal right and left pulmonary artery were obtained on axial (a) and right and left anterior oblique (paracoronal) views $(\mathbf{c}, \mathbf{d})$, respectively

Table 57 References, normal dimensions of the pulmonary arteries in children

\begin{tabular}{|c|c|c|c|}
\hline First author, year & CMR technique & $\mathrm{n}$, male:female & $\begin{array}{l}\text { Age } \\
\text { range } \\
\text { (years) }\end{array}$ \\
\hline Knobel, 2011 [100] & 1.5 T, contrast enhanced CMRA, diameters measured on images perpendicular to the vessel & $41: 28$ & $2-20$ \\
\hline Kutty, 2012 [93] & $\begin{array}{l}1.5 \mathrm{~T} \text {; magnitude image of a through-plane free-breathing phase contrast sequence; cross sectional } \\
\text { area calculated based on measurements of the maximal external aortic diameter perpendicular to } \\
\text { the vessel and perpendicular to the maximal diameter obtained midway between the level of the } \\
\text { pulmonary valve and the bifurcation of the branch pulmonary arteries }\end{array}$ & $55: 50$ & $4-20$ \\
\hline
\end{tabular}

n number of study subjects

A number of pulse sequence parameters can affect normal values. For MOLLI pulse sequences the number of inversions, number of images following each inversion, and number of recovery beats between inversion pulses, and the flip angle affect normal values [101]. Furthermore, the type of inversion pulses, which may be vendor specific can also affect $\mathrm{T} 1$ values. 
Table 58 Normal diameters of the pulmonary arteries in children measured on a contrast enhanced CMRA according to reference [100]

\begin{tabular}{lll}
\hline Site & Predicted diameter $(\mathbf{m m})$ & $\begin{array}{c}\text { SD of } \\
\text { residuals } \\
\text { (mm) }\end{array}$ \\
\hline Main pulmonary artery (axial) & & 2.72 \\
Main pulmonary artery (sagittal) & $4.85+13.43^{*} \mathrm{BSA}^{0.5}$ & 2.01 \\
Proximal right pulmonary artery (axial) & $1.04+17.07^{*} \mathrm{BSA}^{0.5}$ & 1.65 \\
Distal right pulmonary artery (axial) & $2.63+9.19^{*} \mathrm{BSA}^{0.5}$ & 1.49 \\
Proximal right pulmonary artery (RAO) & $3.9+6.25^{*} \mathrm{BSA}^{0.5}$ & 1.76 \\
Distal right pulmonary artery (RAO) & $-0.69+14.3^{*} \mathrm{BSA}^{0.5}$ & 1.6 \\
Proximal left pulmonary artery (axial) & $-1.08+14.62^{*} \mathrm{BSA}^{0.5}$ & 1.37 \\
Distal left pulmonary artery (axial) & $1.7+11.27^{*} \mathrm{BSA}^{0.5}$ & 1.51 \\
Proximal left pulmonary artery (LAO) & $-0.1+11.89^{*} \mathrm{BSA}^{0.5}$ & 1.88 \\
Distal left pulmonary artery (LAO) & $-2.13+16.82^{*} \mathrm{BSA}^{0.5}$ & 1.5 \\
\hline Dimm & $-2.08+13.64^{*} \mathrm{BSA}^{0.5}$ & \\
\end{tabular}

Diameters measured perpendicular to the vessel (Fig. 14).

Fitting model for regression: diameter $=a+b * B S A^{0.5}$

$\mathrm{z}$-score $=$ (measured diameter - predicted diameter)/SD of residuals; lower and upper limits correspond to a $\mathrm{z}$-score of -2 and 2.

$B S A$ body surface area, $S D$ standard deviation, $R A O$ right anterior oblique view (paracoronal, parallel to right pulmonary artery; Fig. 14), LAO left anterior oblique view (paracoronal, parallel to left pulmonary artery; Fig. 14).

Table 59 Normal pulmonary artery area measured on phase contrast cine images according to reference [93]

\begin{tabular}{lc}
\hline Site & Predicted area $\left(\mathbf{c m}^{2}\right)$ \\
\hline Ascending aorta & $-0.2880+3.386^{*} \mathrm{BSA}$
\end{tabular}

Cross sectional area calculated based on measurements of the maximal external aortic diameter perpendicular to the vessel and perpendicular to the maximal diameter obtained midway between the level of the pulmonary valve and the bifurcation of the branch pulmonary arteries.

BSA body surface area

The aforementioned factors contribute to the large heterogeneity of published reference ranges. Heterogeneity in published values are present even if the same manufacturer scanner was used at the same field strength with the same pulse sequence [101]. It is thus imperative to standardize local pulse sequences and sequence parameters, and to follow current consensus guidelines for establishing site specific reference ranges [102]. In contrast to other CMR parameters, the SCMR has indicated that literature normal values of $\mathrm{T} 1$ relaxation times should not serve as absolute reference values, but rather than sitespecific reference ranges should be established [102].

\section{CMR analysis methods}

T1 maps are based on pixel-wise quantification of longitudinal relaxation from the T1-weighted source images. The native $\mathrm{T} 1$ relaxation time, expressed in milliseconds (ms), is a composite measurement reflecting the signal from water within multiple compartments within the myocardium including myocytes, the blood pool, and the interstitial space [113]. Under assumptions of an equilibrium of gadolinium concentrations between the blood pool and interstitium, pre and post contrast blood and myocardial $\mathrm{T} 1$ values can be used to quantify the partition coefficient of gadolinium which when multiplied by (1-hematocrit) quantifies the fractional volume of the extracellular space. This ECV is expressed as a percentage [114].

Offline post-processing involves manually tracing endocardial and epicardial contours [109, 115] (Fig. 15) or placing a region of interest within the septal myocardium. Inclusion of blood pool or adjacent tissue should be carefully avoided. Motion correction is generally used to correct undesired breathing motion. However, motion correction can only correct for in-plane motion and not through-plane motion. All methods, therefore, are vulnerable to partial volume effects.

\section{Demographic parameters}

In one large study, there was no relationship of age to myocardial or blood native $\mathrm{T} 1$ in male subjects aged 11-69 years [116]. In female subjects there was a trend of lower native T1 with increased age (e.g. approximately 20 ms lower for females less than 45 years vs. those greater than 45 years) [116]. Female subjects $<45$ years of age had a consistently higher native T1 then males, but after this age there was no difference in native $\mathrm{T} 1$ by gender [116]. However, other studies have failed to demonstrate a significant trend in native $\mathrm{T} 1$ with age or gender [117]. For ECV measurement, ECV is reported to be higher in females than males, but data are conflicting regarding the relationship of ECV with age [110, 118]. 


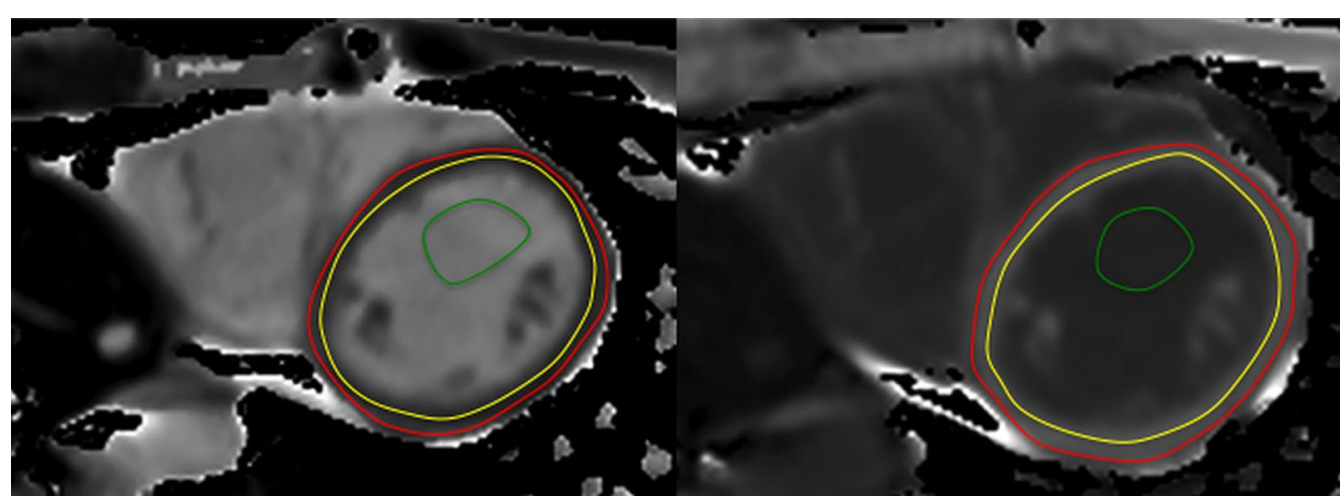

Fig. 15 T1 maps with measurements. T1 map pre- (a) and post-contrast (b) with left ventricular endocardial and epicardial contours according to reference [119]

The above relationships were formally assessed in a recent meta-analysis [101]. Overall, there was no significant association between native $\mathrm{T} 1$ and age or percent of male participants at either $1.5 \mathrm{~T}$ or $3 \mathrm{~T}$. However, there was a significant effect of gender with studies including more females on average having higher reported ECV values [101].

\section{Studies included in this review}

SCMR guidelines indicate each site should establish their own site specific reference ranges for T1 mapping parameters. In the absence of such data however, the weighted mean values and reference ranges for native T1 time and ECV based on publications of at least 40 healthy subjects extracted from Table 60 are summarized in Table 61.

\section{Normal values of myocardial $\mathrm{T} 2$ relaxation times CMR acquisition parameters}

$\mathrm{T} 2$ relaxation time is the exponential time constant for the relaxation of transverse magnetization. To determine myocardial T2 time, a relaxation curve is constructed based on a CMR multi-echo pulse sequence. The most-commonly used technique utilizes a T2-preparation module followed by either a single-shot bSSFP or GRE readout [150, 151]. This technique typically acquires 3 source images with effective echo times of 0 , 30 and $60 \mathrm{~ms} .3-4$ heart-beats are allowed for T1 relaxation between acquisition of source images, and data is acquired during a single breath-hold of 9-12 heart-beats. Inadequate time for complete T1-relaxation between source images can cause a T1-based bias in the T2 maps. The bSSFP technique has higher signal-to-noise but is more susceptible to off-resonance artifacts than the GRE technique. Other techniques are based on turbo-spin echo (TSE) or GRadient And Spin Echo (GRASE) acquisition modes. TSE sequences consist of a $90^{\circ}$ excitation followed by a train of $180^{\circ}$ refocusing pulses, with each focusing pulse producing a spin-echo with a different echo time (TE). By creating images corresponding to each echo time in the train, T2 maps can be produced by fitting the T2-signal decay equation. TSE sequences are robust to off-resonance, but they can suffer from inaccuracies due to imperfect $180^{\circ}$ pulses which result in stimulated-echo contamination. GRASE sequences consist of a $90^{\circ}$ excitation followed by a train of $180^{\circ}$ pulses which produce a spin echo, and 2-4 gradient echoes. This technique is more efficient than TSE but is subject to similar biases as the TSE technique, and additionally is more sensitive to off-resonance effects due to the presence of gradient echoes and longer spacing between $180^{\circ}$ pulses. Of note, performing multiple TSE sequences with different effective-TEs are inaccurate for determining T2 and are not recommended.

\section{Factors affecting $\mathrm{T} 2$ relaxation time}

There are a number of factors which can affect the measurement of normal T2 values. Field strength has a small effect on T2 values, with $3 \mathrm{~T}$ scans typically having T2 values that are $\sim 6 \mathrm{~ms}$ shorter than those obtained on $1.5 \mathrm{~T}$ scanners [152]. There are differences in measured T2 based on technical factors such as the type of pulsesequence used and the vendor. The T2-preparation pulse may be sensitive to off-resonance and B1 inhomogeneity effects; these effects are more severe at $3 \mathrm{~T}$. T2-preparation based on adiabatic radiofrequency (RF)-pulses have been shown to lessen these effects at $3 \mathrm{~T}$. TSE and GRASE sequences are sensitive to specifics of the RFpulses which are vendor and implementation dependent. Similar to T1 mapping, it is imperative to standardize local pulse sequence parameters. As for T1 mapping, site-specific reference ranges should be established. 
Table 60 References, native myocardial T1 relaxation time and extracellular volume fraction (ECV)

\begin{tabular}{|c|c|c|c|}
\hline First author, year & CMR technique & n, male:female & $\begin{array}{l}\text { Age range or } \\
\text { mean } \pm S D \\
\text { (years) }\end{array}$ \\
\hline Fontana, 2012 [120] & 1.5 T, Siemens, ShMOLLI, ECV & $27: 23$ & $47 \pm 17$ \\
\hline Kellman, 2012 [121] & 1.5 T, Siemens, MOLLI, native T1 and ECV & $30: 32$ & $47 \pm 17$ \\
\hline Piechnik, 2013 [116] & 1.5 T, Siemens, ShMOLLI, native T1 & 169:173 & $11-69$ \\
\hline Sado, 2013 [122] & 1.5 T, Siemens, ShMOLLI, native T1 & $30: 37$ & $24-88$ \\
\hline Ferreira, 2014 [123] & 1.5 T, Siemens, ShMOLLI, native T1 & $37: 13$ & $41 \pm 13$ \\
\hline Fontana, 2014 [124] & 1.5 T, Siemens, ShMOLLI, native T1 & $17: 35$ & $46 \pm 15$ \\
\hline Liu, 2014 [125] & 3T, Siemens, MOLLI, native T1 & $38: 54$ & $27-44$ \\
\hline Puntmann, 2014 [126] & 3T, Philips, MOLLI, native T1 & 47 (total) & $--^{\mathrm{a}}$ \\
\hline Reiter, 2014 [118] & 1.5 T, Siemens, MOLLI, native T1 & $20: 20$ & $20-35$ \\
\hline aus dem Siepen, 2015 [127] & 1.5 T, Philips, MOLLI, native T1 and ECV & $37: 19$ & $52 \pm 9$ \\
\hline Banypersad, 2015 [128] & 1.5 T, Siemens, ShMOLLI, native T1 and ECV & $25: 29$ & $46 \pm 15$ \\
\hline Edwards, 2015 [129] & 1.5 T, Siemens, MOLLI, native T1 and ECV & $24: 19$ & $57 \pm 10$ \\
\hline Fontana, 2015 [130] & 1.5 T, Siemens, ShMOLLI, native T1 and ECV & $21: 26$ & $24-69$ \\
\hline Treibel, 2015 [131] & 1.5 T, Siemens, ShMOLLI, native T1 and ECV & $26: 24$ & $28-69$ \\
\hline Goebel, 2015 [132] & 1.5 T, Siemens, MOLLI, native T1 & $31: 23$ & $18-63$ \\
\hline Gormeli, 2016 [133] & 3T, Siemens, MOLLI, native T1 & $26: 15$ & $24 \pm 4$ \\
\hline Hinojar, 2016 [134] & 3 T, Philips, MOLLI, native T1 & $9: 37$ & $42 \pm 15$ \\
\hline Ntusi, 2016 [135] & 1.5 T, Siemens, ShMOLLI, native T1 & $53: 39$ & $44 \pm 10$ \\
\hline Rauhalammi, 2016 [136] & 1.5 T and $3 \mathrm{~T}$, Siemens, MOLLI, native T1 & 43: 41 & $45 \pm 18$ \\
\hline Costello, 2017 [137] & $3 \mathrm{~T}$, Siemens, ShMOLLI, native T1 and ECV & $29: 28$ & $48 \pm 15$ \\
\hline Avitzur, 2018 [138] & 3T, Siemens, ShMOLLI, native T1 & $83: 57$ & $54 \pm 9$ \\
\hline Doerner, 2018 [139] & 1.5 T, Philips, MOLLI, native T1 and ECV & $30: 20$ & $39 \pm 17$ \\
\hline Guo, 2018 [140] & 3T, Philips, MOLLI, native T1 and ECV & 18:32 & $36 \pm 16$ \\
\hline Ridouani, 2018 [141] & 1.5 T, Siemens, MOLLI, native T1 & $20: 20$ & $40 \pm 12$ \\
\hline Rosmini, 2018 [142] & 1.5 T, Siemens, MOLLI and ShMOLLI, native T1 and ECV & 49:45 & $20-76$ \\
\hline Shang, 2018 [143] & $3 \mathrm{~T}$, Siemens, MOLLI, ECV & 45 (total) & $-^{\mathrm{a}}$ \\
\hline Yang, 2018 [144] & 3T, Siemens, MOLLI, native T1 and ECV & $18: 26$ & $33 \pm 16$ \\
\hline Granitz, 2019 [145] & 1.5 T and $3 \mathrm{~T}$, Philips, MOLLI, native T1 & $26: 32$ & $\begin{array}{l}42 \pm 13 \\
\text { (male) } \\
40 \pm 14 \\
\text { (female) }\end{array}$ \\
\hline Imran, 2019 [146] & 1.5 T, Philips, MOLLI, native T1 & $26: 25$ & $46 \pm 14$ \\
\hline Lehmonen, 2019 [147] & 1.5 T, Siemens, ShMOLLI, native T1 & 46 (total) & $46 \pm 9$ \\
\hline Vijapurapu, 2019 [148] & 1.5 T, Siemens, ShMOLLI, native T1 & $40: 37$ & $49 \pm 14$ \\
\hline Wan, 2019 [149] & 3T, Siemens, MOLLI, native T1 and ECV & $20: 20$ & $56 \pm 9$ \\
\hline
\end{tabular}

$n$ number of study subjects, MOLLI modified look locker inversion-recovery, ShMOLLI short MOLLI, $T 1 \mathrm{~T} 1$ relaxation time, ECV extracellular volume fraction

${ }^{\text {a }}$ Not provided in original publication

\section{CMR analysis methods}

T2 is the relaxation time (in milliseconds) of the transverse magnetization. Similar to T1 assessment, to generate parametric maps of T2, the source images typically need to be aligned using non-rigid registration. Again, these techniques can correct for in-plane motion but not through-plane motion. Both off-line and on-line techniques have been used as for T1 mapping.

\section{Demographic parameters}

Published data on T2 values have sample sizes smaller than those of T1 methods. Thus, effects of demographic parameters in relationship to T2 times are not well established. One paper using GRASE demonstrated a slightly higher native $\mathrm{T} 1$ in females as compared to males $(56.7 \mathrm{~ms}$ vs $54.6 \mathrm{~ms} ; \mathrm{p}=0.008)$ at $1.5 \mathrm{~T}$ but no difference at $3 \mathrm{~T}$. No significant differences 
Table 61 Native myocardial T1 relaxation time and extracellular volume fraction (ECV)

\begin{tabular}{|c|c|c|c|c|c|c|c|}
\hline Parameter & $\mathrm{FS}(\mathrm{T})$ & Vendor & Technique & $\mathbf{n}$ & Mean $_{p}$ & $S D_{p}$ & LL-UL $^{m}$ \\
\hline \multirow[t]{6}{*}{ Native T1 time (ms) } & 1.5 & Siemens & MOLLI & $417^{a}$ & 972 & 43 & $885-1059$ \\
\hline & 1.5 & Siemens & ShMOLLI & $971^{b}$ & 960 & 29 & $903-1017$ \\
\hline & 1.5 & Philips & MOLLI & $215^{c}$ & 989 & 42 & $905-1073$ \\
\hline & 3 & Siemens & MOLLI & $301^{d}$ & 1196 & 47 & $1103-1290$ \\
\hline & 3 & Siemens & ShMOLLI & $197^{e}$ & 1130 & 55 & $1021-1240$ \\
\hline & 3 & Philips & MOLLI & $201^{f}$ & 1097 & 66 & $964-1230$ \\
\hline \multirow[t]{6}{*}{ ECV (\%) } & 1.5 & Siemens & MOLLI & $199^{9}$ & 26 & 3 & $20-32$ \\
\hline & 1.5 & Siemens & ShMOLLI & $295^{h}$ & 27 & 3 & $21-33$ \\
\hline & 1.5 & Philips & MOLLI & $56^{i}$ & 23 & 3 & $17-29$ \\
\hline & 3 & Siemens & MOLLI & $129^{j}$ & 26 & 3 & $20-32$ \\
\hline & 3 & Siemens & ShMOLLI & $57^{k}$ & 25 & 2 & $20-29$ \\
\hline & 3 & Philips & MOLLI & $100^{\prime}$ & 26 & 5 & $16-36$ \\
\hline
\end{tabular}

$E C V$ extracellular volume fraction, $F S$ field strength, $T$ Tesla, $n$ number of study subjects included in the weighted mean values, mean ${ }_{p}$ pooled weighted mean, $S D_{p}$ pooled standard deviation, LL lower limit, UL upper limit, MOLLI modified look locker inversion-recovery, ShMOLLI short MOLLI, Siemens Siemens Medical Solutions, Erlangen, Germany, Philips Philips Healthcare, Best, The Netherlands

a Pooled weighted values from references [118, 121, 129, 132, 136, 141, 142]

${ }^{b}$ Pooled weighted values from references [116, 122-124, 128, 130, 131, 135, 142, 147, 148]

' Pooled weighted values from references $[127,139,145,146]$

d Pooled weighted values from references [125, 133, 136, 144, 149]

e Pooled weighted values from references $[137,138]$

${ }^{f}$ Pooled weighted values from references [126, 134, 140, 145]

${ }^{g}$ Pooled weighted values from references [121, 129, 142]

h Pooled weighted values from references [124, 128, 130, 131, 142]

'Values from reference [127]

j Pooled weighted values from references [143, 144, 149]

${ }^{k}$ Values from reference [137]

' Pooled weighted values from references $[139,140]$

${ }^{m}$ Calculated as mean m $\pm 2 * \mathrm{SD}_{\mathrm{p}}$

in T2 were seen as a function of age [145]. Another study showed no difference between male and female subjects when controlling for age, but did see a trend of lower T2 with increasing age [153]. Another study using T2-prepared bSSFP at $3 \mathrm{~T}$ demonstrated no significant differences in T2 by age or gender [154].

\section{Studies included in this review}

SCMR guidelines indicate each site should establish their own site specific reference ranges for T2 mapping parameters. In the absence of such data however, the weighted mean values and reference ranges for $\mathrm{T} 2$ on publications of at least 40 healthy subjects (combined males and females) are shown in Table 62.

\section{Normal values of myocardial T2* relaxation time CMR acquisition parameters}

Quantification of the T2* relaxation time plays an important role for estimation of myocardial iron overload [156]. T2* time is also altered in myocardial necrosis and hemorrhage [102]. For quantification of the myocardial
T2* time, the gradient-echo $\mathrm{T} 2 *$ technique with multiple increasing echo times is preferred over the spin-echo T2 technique due to a greater sensitivity to iron deposition [157-159]. According to the current consensus statement by the SCMR, a dark-blood multi-echo gradient echo sequence with 8 equally spaced echoes between 2 and $18 \mathrm{~ms}$ should be used for T2*-mapping at $1.5 \mathrm{~T}$ [102] Usually a single-breath hold technique is used. Normal values and a grading system for myocardial iron overload are available for $1.5 \mathrm{~T}$ [158].

\section{CMR analysis methods}

Gradient-echo T2* images are vulnerable to distortions of the local magnetic field e.g. by air-tissue interfaces. The myocardial septum is surrounded by blood on both sides, so susceptibility differences are less than in the lateral wall with improved image quality on $\mathrm{T} 2 *$ images. Therefore, $\mathrm{T}^{*}$ measurements are obtained by placing a region of interest on the interventricular septum of a midventricular short axis slice [102, 159] (Fig. 16). 
Table 62 Myocardial T2 relaxation time (ms)

\begin{tabular}{|c|c|c|c|c|c|c|c|c|}
\hline First author, year & $\mathrm{FS}(\mathrm{T})$ & Vendor & Technique & n, male:female & Age range or mean \pm SD (years) & Mean & SD & $\mathrm{LL}_{-} \mathrm{UL}^{\mathrm{a}}$ \\
\hline Wassmuth, 2013 [155] & 1.5 & Siemens & T2P bSSFP & $60: 13$ & $20-70$ & 55 & 5 & $45-65$ \\
\hline Wassmuth, 2013 [155] & 1.5 & Siemens & FLASH & $60: 13$ & $20-70$ & 52 & 5 & $42-62$ \\
\hline Hinojar, 2016 [134] & 3 & Philips & GSE & $9: 37$ & $42 \pm 15$ & 45 & 4 & $37-53$ \\
\hline Ridouani, 2018 [141] & 1.5 & Siemens & T2P bSSFP & $20: 20$ & $40 \pm 12$ & 51 & 3 & $45-57$ \\
\hline Granitz, 2019 [145] & 1.5 & Philips & GSE & $26: 32$ & $40 \pm 14$ (male), $42 \pm 13$ (female) & 56 & 3 & $50-62$ \\
\hline Granitz, 2019 [145] & 3 & Philips & GSE & $26: 32$ & $40 \pm 14$ (male), $42 \pm 13$ (female) & 52 & 3 & $46-58$ \\
\hline
\end{tabular}

FS field strength, $T$ Tesla, $n$ number of study subjects, SD standard deviation, LL lower limit, UL upper limit, T2PT2 preparation, $b S S F P$ balanced steady state free precession, FLASH fast low angle shot, GSE gradient spin echo, Siemens Siemens Medical Solutions, Erlangen, Germany, Philips Philips Healthcare, Best, The Netherlands

${ }^{\text {a }}$ Calculated as mean $\pm 2 * S D$

$\mathrm{T} 2 *$ times are frequently reported as relaxation rate, representing the reciprocal of the time constant and calculated as R2 $* 1000 / \mathrm{T} 2 *$. The units of R2* is s ${ }^{-1}$ [159]. Cardiac iron concentration can be calculated from $\mathrm{T} 2^{*}$ values by the following equation: $[\mathrm{Fe}]=45 /\left(\mathrm{T} 2^{*}\right)^{1.22}$, where $[\mathrm{Fe}]$ is the cardiac iron concentration in milligrams per gram dry weight and $\mathrm{T} 2 \%$ in milliseconds [160].

\section{Demographic parameters}

T2* of the myocardium is not related to age [161]. To our knowledge the relationship between other demographic parameters and $\mathrm{T} 2 *$ has not been assessed.

\section{Studies included in this review}

The mean T2* of the myocardium (interventricular septum) is approximately $36 \mathrm{~ms}$ [161] at $1.5 \mathrm{~T}$ using a multiecho GRE sequence. T2* $>20 \mathrm{~ms}$ is considered within the range of normal.

Depending on the risk to develop heart failure as a consequence of myocardial iron overload, a grading system for disease severity has been published (Table 63) [102, $156,162]$.

\section{Regional measurements and cardiac strain CMR acquisition parameters}

A number of imaging methods have been developed to acquire cardiac strain information from cine CMR. These methods include tagged cine CMR, PC-CMR, velocity encoded CMR, displacement encoding with stimulated echoes (DENSE), and strain-encoding (SENC) $[163,164]$. Tagged CMR is a widely validated reproducible tool for strain estimation. The method is used in clinical studies and is considered the reference standard for assessing regional function $[165,166]$. Recently feature-tracking CMR (FT-CMR) has been increasingly reported due to compatibility with existing cine CMR images [167].

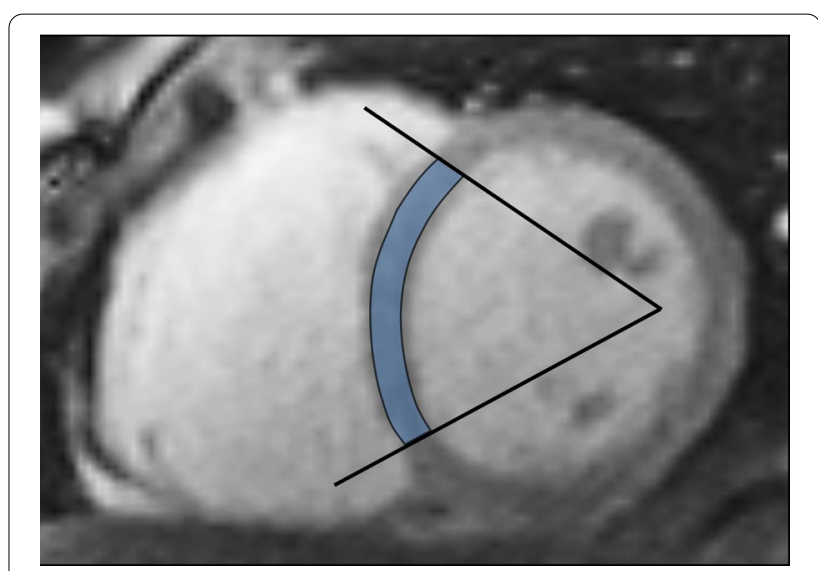

Fig. 16 Measurements of myocardial T2* are obtained in the septum

Table 63 Grading of iron overload based on T2* measurements at 1.5 T according to $[102,156,162]$

\begin{tabular}{ll}
\hline Iron overload & T2* (ms) \\
\hline Normal & $>20$ \\
Iron overload & $10-20$ \\
Severe iron overload & $<10$ \\
\hline
\end{tabular}

\section{CMR analysis methods}

Cardiac strain is a dimensionless measurement of the deformation that occurs in the myocardium. Cardiac strain can be reported as three normal strains (circumferential, radial, and longitudinal) and six shear strainsthe angular change between two originally mutually orthogonal line elements, with the more clinically investigated shear strain and the circumferential-longitudinal shear strain (also known as torsion). They can also be computed as fiber and cross-fiber strains which require anatomical knowledge of fiber architecture, or 


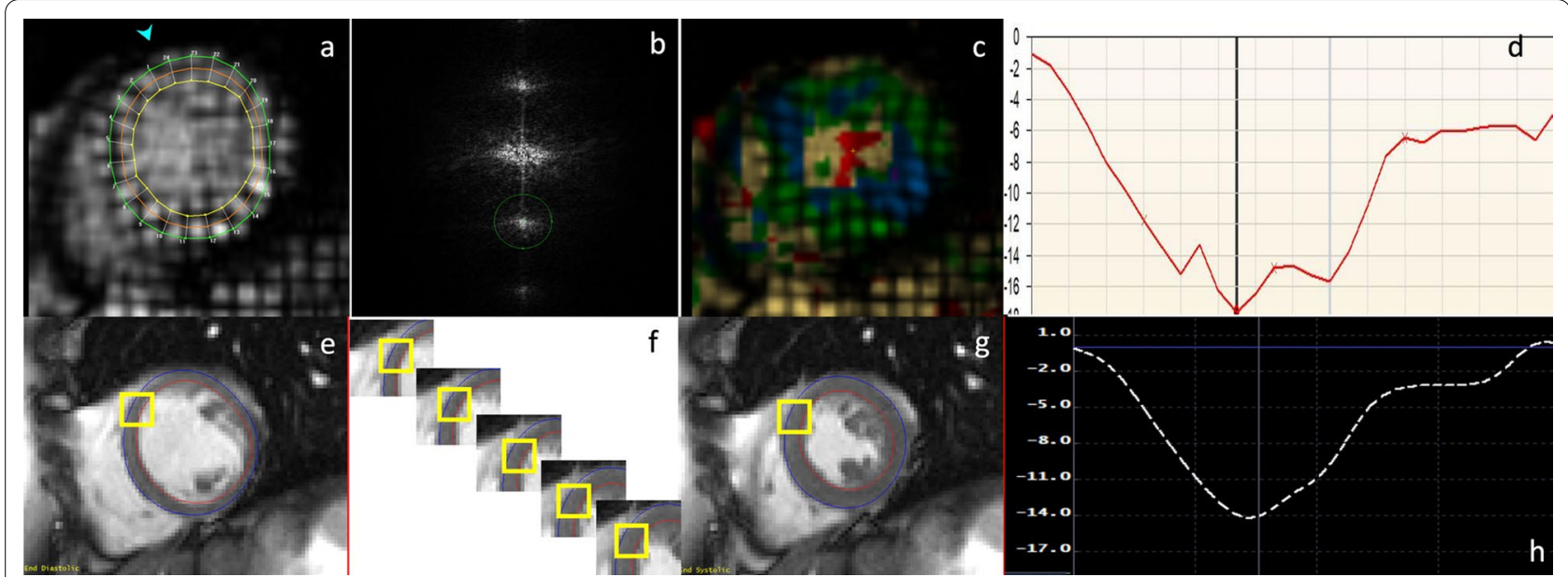

Fig. 17 Illustration of strain computation using the Harmonic Phase (HARP) tool on tagged CMR images (a-d) and from feature tracking on cine CMR images (e-h). In HARP, first a semi-automated frequency analysis of the tagged CMR image (a) is performed to identify the harmonic peaks in each of the tag directions (b), filters are then applied to isolate the peaks and obtain the corresponding phase maps from which Eulerian strain maps (c) can be computed. Subplot (d) shows the strain curve at the mid-ventricular level for an asymptomatic volunteer obtained based on tracking of the user-defined mesh (a). In feature tracking of cine CMR images, endo- and epicardial contours are drawn at end-diastole (e) or end-systole $(\mathbf{g})$. A characteristic pixel pattern in the order of a few millimeters squared is identified as a template. The software then tries to discern a similar pattern in the subsequent frame from which displacement of the pixels is computed (f). This is repeated through the entire cycle to obtain displacement from which strain is computed. Subplot (h) shows the strain curve at the mid-ventricular level computed from feature tracking. The tagged and cine CMR images and the strain curves were from the same participant

as principal strains along the principal stretching and shortening directions [168]. Here, we concentrate on the widely reported circumferential and longitudinal strains. Although frequently reported, radial strain is less reproducible because of the reduced resolution of imaging in the radial direction as opposed to the circumferential or longitudinal directions.

There are a number of different methods to quantify strain: registration methods, feature-based tracking methods, deformable models, Gabor Filter Banks, optic flow methods, harmonic phase analysis (HARP) [169], and local sine wave modeling (SinMod) [163]. Technical review papers for these methods can be found in the following literature [167, 170-172].

HARP is one of the most widely reported and validated methods for analyzing tagged CMR for cardiac strain, in part due to its large scale use in the MESA study [169, 173]. Strain patterns are reported according to the 16 or 17 segment AHA model. Consistent manual tracing of the endocardial and epicardial contours is necessary to reproducible strain results. With tagged CMR, midwall strain is preferred to epicardial and endocardial strain to maximize the amount of tagging data available for strain calculations [172, 174]. With HARP analysis such as that used in the MESA trial [169], careful selection of the first harmonic is necessary. Figure 17a shows an outline of tagged CMR analysis using HARP.
FT-CMR has shown diagnostic and prognostic utility across a variety of pathologies. Currently, FT-CMR software from TomTec (TomTec Imaging Systems, Unterschleissheim, Germany), QStrain (Medis Medical Imaging Systems, Leiden, The Netherlands) and CVI42 (Circle Cardiovascular Imaging Inc., Calgary, Canada) are widely used in clinical research for calculation of LV strains. Similar to tagged CMR from HARP, strains are reported in the 16 or 17 segment models.

Segmentation of the myocardium (either semi-automated or completely automated) at the start of the cardiac cycle is an essential first step across all software. The software records a characteristic pixel pattern (an area of pixels typically in the order of $10-15 \mathrm{~mm}^{2}$ ) in the reference frame; an area with an identical pixel pattern is recognized in the next frame that maximizes certain similarity metrics $[167,175]$. This procedure is repeated for all pixels in each image and for each frame to track the borders throughout the whole cardiac cycle with constraints for smoothness of the deformation field applied. FT-CMR tracking quality is largely governed by the quality and resolution of the pixels at the endocardial and epicardial borders which are feature-rich as compared to the mid-myocardium. This method has been extended to volume data by tracking voxels to obtain 3D FT-CMR [176]. Figure 17b shows an outline of the concept underlying strain analysis by FT-CMR. 
Table 64 References, myocardial strain

\begin{tabular}{|c|c|c|c|}
\hline First author, year & CMR technique & n, male:female & Age range (years) \\
\hline Neizel, 2009 [182] & 1.5 T, 3 short axis images, tagged CMR (SPAMM), tagged resolution $7 \mathrm{~mm}$; HARP method & $40: 35$ & $22-69$ \\
\hline Augustine, 2013 [177] & $\begin{array}{l}1.5 \text { T, short axis stack bSSFP, } 2,3 \text {, and } 4 \text { chamber bSSFP; feature tracking (TomTec } \\
\text { software) }\end{array}$ & $54: 62$ & $(30 \pm 8)^{a}$ \\
\hline Venkatesh, 2015 [183] & 1.5 T, 3 short axis images, tagged CMR (SPAMM), tagged resolution $7 \mathrm{~mm}$, HARP method ${ }^{\mathrm{b}}$ & $46: 83$ & $45-84$ \\
\hline Andre, 2015 [179] & 1.5 T, short axis stack, 2, 3, and 4 chamber bSSFP; feature tracking (TomTec software) & $75: 75$ & $21-71$ \\
\hline Cai, 2017 [66] & $3 \mathrm{~T}$, short axis stack, 2, 3, and 4 chamber bSSFP; feature tracking (CVI42 software $\left.{ }^{\mathrm{d}}, 2 \mathrm{D}\right)$ & $91: 89$ & $20-69$ \\
\hline Liu, 2018 [26] & $\begin{array}{l}\text { 1.5 T, short axis stack bSSFP, 2, 3, and } 4 \text { chamber bSSFP; feature tracking (CVIR42 } \\
\left.\text { software }{ }^{d}, 3 D\right)\end{array}$ & $50: 50$ & $20-70$ \\
\hline Peng, 2018 [176] & $\begin{array}{l}1.5 \text { T and } 3 \text { T, short axis stack, 2, 3, and } 4 \text { chamber bSSFP; feature tracking (QStrain } \\
\text { software })^{e} \text { ) }\end{array}$ & $75: 75$ & $18-82$ \\
\hline
\end{tabular}

$n$ number of study subjects, SPAMM spatial modulation of magnetization, HARP harmonic phase, bSSFP balanced steady state free precession

${ }^{a}$ Mean $\pm S D$ (age-range not provided in original publication)

${ }^{\mathrm{b}}$ HARP commercial, Diagnosoft, Palo Alto, CA, USA

'TomTec Imaging Systems, Unterschleissheim, Germany

${ }^{d}$ CMI42, Circle Cardiovascular Imaging Inc., Calgary, Canada

e QStrain, Medis Medical Imaging Systems, Leiden, The Netherlands

\section{Demographic parameters}

Using both tagged CMR and FT-CMR, several studies report greater age is associated with decrease in peak circumferential or longitudinal shortening [176-178]. In tagged CMR and a few FT-CMR reports, gender also affects normal values. Cardiac strain values for women are higher than those of men [66, 176, 179-181]. However, some FT-CMR reports showed no association of circumferential or longitudinal strains with age or gender $[26,177]$.

\section{Studies included in this review}

Several studies have presented cohorts for determining normal LV strain. For the purpose of this review, only cohorts of 40 or more normal subjects using SPAMM (spatial modulation of magnetization tagging) or FTCMR have been included. Inclusion criteria include a full description of the subject cohort (including the analysis methods used), age and gender of subjects. Table 64 represents a summary of publications reporting normal values for strain that fit the criteria. We have only included reference values for global values of strain as the interreader and inter-study reproducibility of regional strain values vary widely between published reports.

With tagged CMR, normal midwall circumferential strain values are relatively comparable between studies [182, 183] (Table 65). With 2D FT-CMR, small differences between published results exist for reference values, probably due to inter-vendor differences $[26,66$, $177,179]$. The reference ranges of normal circumferential strains from FT-CMR (Table 66) are comparable to those obtained from tagged CMR (Table 65). Strain values are traditionally reported as more negative values meaning greater contractility. For both global circumferential and global longitudinal strain, a strain value of approximately $-14 \%$ is the limit of normal; values more positive than this are considered to be abnormal.

Given the inclusion criteria noted above, one publication [176] used 3D FT-CMR (Table 67). The mean values and reference ranges were lower compared to 2D FTCMR and tagged-CMR.

\section{Myocardial perfusion}

Myocardial perfusion has been quantified with T1-weighted dynamic imaging during the first pass of a contrast bolus by semi-quantitative methods that derive dimensionless indices (e.g. the upslope of myocardial signal intensity changes during initial contrast enhancement). Alternatively, absolute estimates of MBF may be determined (in units of $\mathrm{ml}$ per $\mathrm{g}$ of tissue and per minute $(\mathrm{ml} / \mathrm{g} / \mathrm{min})$ ). To derive absolute measures of blood flow, the CMR signal intensity changes must be converted to be linearly proportional to contrast concentration. This assumption may not hold true at high contrast concentrations (e.g. in the blood pool). Instead, low-dose bolus injections of contrast agent (e.g. $<\sim 0.04 \mathrm{mmol} / \mathrm{kg}$ of Gd-DTPA) with saturation correction [184] or special pulse sequences are used for CMR perfusion (e.g. "dualsequence" [185], dual-bolus techniques [186, 187]) to provide linear measures of gadolinium concentration.

\section{CMR acquisition parameters}

Normal values for quantitative myocardial perfusion measures have been obtained by ECG-gated, T1-weighted dynamic imaging during the first pass of an injected contrast bolus using gradient-echo image 


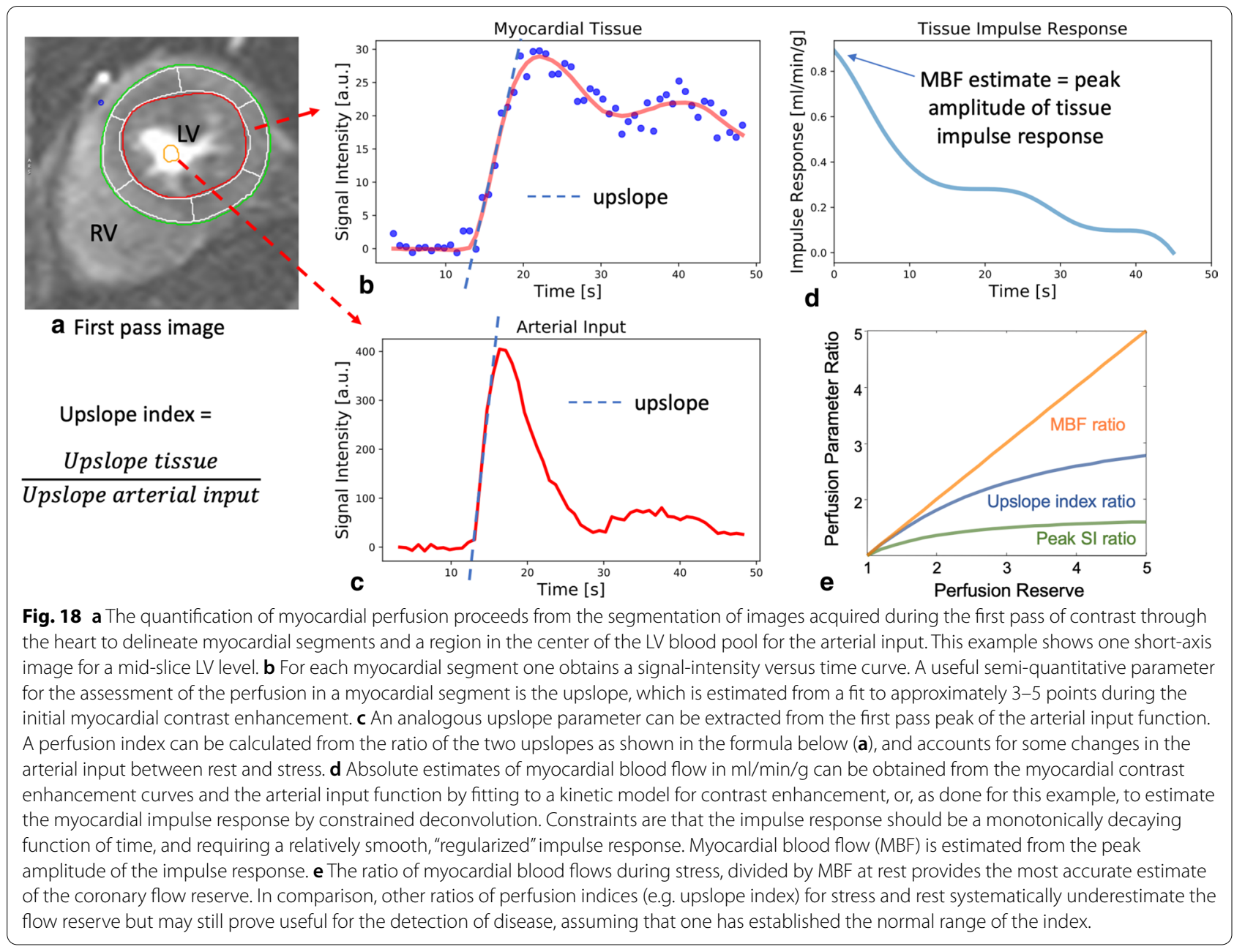

readouts without or with bSSFP. Echo-times are kept as short as possible for any of these image acquisition methods to minimize $\mathrm{T} 2 \%$-related signal loss. T1-weighting is generally maximized by using saturation-recovery magnetization preparations. Semi-quantitative parameters depend on contrast dosage and injection protocol, sequence technique and acquisition parameters. The normal ranges for semi-quantitative parameters should therefore only be used as reference when the same

Table 65 Left ventricular global peak circumferential strain using tagging

\begin{tabular}{llccl}
\hline Parameter & $\mathbf{n}$ & Mean $_{\mathbf{p}}$ & $\mathbf{S D}_{\mathbf{p}}$ & LL-UL $^{\mathbf{a}}$ \\
\hline Circumferential strain (\%) & 204 & -20.1 & 3.0 & -26.0 to -14.2 \\
\hline
\end{tabular}

Pooled weighted values from references [182, 183]

$n$ number of study subjects included in the weighted mean values, mean pooled weighted mean, $S D_{p}$ pooled standard deviation, $L L$ lower limit, UL upper limit

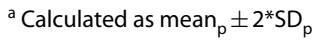

protocol settings are employed. MBF (in units of $\mathrm{mL} /$ $\mathrm{min} / \mathrm{g}$ ) should be independent of the specific perfusion imaging protocol settings. However, a specific technique may still introduce a bias to under or over-estimate MBF.

In clinical use, myocardial perfusion imaging is generally performed at rest and during vasodilator stress. Adenosine and regadenoson are currently the most frequently used pharmacological stress agents for myocardial perfusion imaging and have supplanted dipyridamole in this role. Adenosine and regadenoson have similar hemodynamic effects on coronary artery blood flow [188]. The choice of pharmacologic stress agent is mostly determined by considerations of patient comfort, safety and cost. Regadenoson is more expensive, but better tolerated than adenosine. A unique application of myocardial perfusion imaging is its use in combination with the cold-pressor test to assess coronary endothelial function [189, 190]. 
Table 66 Left ventricular global peak circumferential and longitudinal strain for men and women using 2D feature tracking

\begin{tabular}{|c|c|c|c|c|c|c|c|c|}
\hline \multirow[t]{2}{*}{ Parameter } & \multicolumn{4}{|c|}{ Men } & \multicolumn{4}{|c|}{ Women } \\
\hline & $\mathrm{n}$ & Mean $_{p}$ & $S D_{p}$ & $\mathrm{LL}_{-} \mathrm{UL}^{\mathrm{a}}$ & $n$ & Mean $_{p}$ & $S D_{p}$ & LL-UL ${ }^{a}$ \\
\hline Circumferential strain (\%) & 295 & -20.9 & 3.2 & -27.2 to -14.6 & 301 & -22.7 & 3.3 & -29.2 to -16.2 \\
\hline Longitudinal strain (\%) & 295 & -19.4 & 3.3 & -26.1 to -12.7 & 301 & -21.4 & 3.6 & -28.7 to -14.2 \\
\hline
\end{tabular}

Pooled weighted values from references $[66,176,177,179]$

$n$ number of study subjects included in the weighted mean values, mean $_{p}$ pooled weighted mean, $S D_{p}$ pooled standard deviation, $L L$ lower limit, $U L$ upper limit

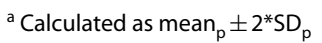

Table 67 Left ventricular global peak circumferential and longitudinal strain using 3D feature tracking according to reference [26]

\begin{tabular}{lcccl}
\hline Parameter & $\mathbf{n}$ & Mean & SD & LL-UL $^{\mathbf{a}}$ \\
\hline Circumferential strain (\%) & 100 & -17.6 & 2.6 & -22.8 to -12.4 \\
Longitudinal strain (\%) & 100 & -14.6 & 2.7 & -20.0 to -9.2 \\
\hline
\end{tabular}

$n$ number of study subjects, SD standard deviation, LL lower limit, UL upper limit

${ }^{\text {a }}$ Calculated as mean $\pm 2 * \mathrm{SD}$

\section{CMR analysis methods}

All quantitative approaches for CMR myocardial perfusion are based on signal-intensity versus time curves that depict the contrast enhancement during the first pass and recirculation of an injected contrast bolus. The myocardial perfusion images are segmented along the endo- and epicardial borders, and the ventricular wall is divided into segments following a standardized segmentation model for cardiac perfusion studies (Fig. 18).

The most widely used semi-quantitative parameter has been the up-slope parameter for initial myocardial contrast enhancement. Because the upslope derives from signalintensity curves with arbitrary units, the value of the upslope depends on the image acquisition settings and on the characteristics of the contrast bolus. For this reason, the myocardial up-slope parameter is generally normalized by the up-slope of the arterial blood pool of the LV to obtain a dimensionless perfusion index. This index is quantified during resting conditions and "stress" at maximal vasodilation (i.e. hyperemia) after infusion of a pharmacological agent (e.g. adenosine). The coronary flow reserve is the ratio of the "stress" index, divided by "rest" index. We refer below to this parameter as the "up-slope" perfusion reserve.

Absolute quantification of the myocardial perfusion reserve entails estimating $\mathrm{MBF}$ in $\mathrm{ml} / \mathrm{min} / \mathrm{g}$. MBF quantification can be based on tracer-kinetic modeling, or by using a deconvolution technique that is based on Zierler's central volume theorem [191]. In either case it is important to have an accurate depiction of the arterial input of contrast to a myocardial region of interest, which in practice is approximated by the arterial contrast enhancement observed in the LV cavity. The myocardial perfusion reserve is estimated as "stress" MBF, divided by "rest" MBF. Nevertheless, hyperemic MBF by itself is also a useful measure of the maximal vasodilator response and its normal range is also provided by some studies in the literature. Rest MBF increases in proportion to the cardiac workload, and the rate-pressure product (RPP) is used as measure of cardiac workload to provide an RPPnormalized MBF value (rest MBF/RPP), whose normal range is narrower in healthy persons than for the rest MBF without any adjustment for RPP.

\section{Demographic parameters}

In the CMR study by Wang et al. [192], rest MBF was higher in women than in men; this agrees with previous studies in healthy subjects using positron emission tomography [193]. Men have a lower hyperemic MBF compared to women, with adjustment for coronary heart disease risk factors [192]. Although male sex carries a higher risk for coronary heart disease, few studies of myocardial perfusion in healthy subjects have considered gender-related differences in MBF. The coronary flow response to the cold-pressor test is also higher in women compared to men [194].

\section{Studies included in this review}

There are two publications reporting reference values of absolute MBF at rest and under pharmacological stress with a sufficient sample size ( $>40$ healthy subjects) (Table 68). The original study published by Wang et al. included subjects from the MESA population with comorbidities such as hypertension and diabetes [192]. However, for the purpose of this review, a re-analysis of [192] was performed for a subset of 99 healthy subjects of the cohort by one of the authors $(\mathrm{MJH})$. Values are given for the entire cohort and for men and women separately. In the other study by Brown et al. reference ranges are presented for the entire cohort of 42 healthy subjects [195]. Although in both studies images were acquired by means of a $\mathrm{T} 1$ weighted saturation recovery prepared single-shot GRE sequences, normal reference ranges 
Table 68 References, normal absolute myocardial blood flow at rest and stress and perfusion reserve in adults and children

\begin{tabular}{|c|c|c|c|}
\hline First author, year & CMR technique & n, male:female & Age mean $\pm S D$ (years) \\
\hline Wang, 2006 [192] a & $\begin{array}{l}\text { 1.5 T, T1 weighted saturation recovery single-shot GRE, at rest and under adenosine } \\
\text { stress }\end{array}$ & $49: 50$ & $59 \pm 11$ \\
\hline Madriago, 2015 [196] & $\begin{array}{l}3 \mathrm{~T}, \mathrm{~T} 1 \text { weighted saturation recovery single-shot GRE, at rest and under adenosine } \\
\text { stress }\end{array}$ & $11: 9$ & $8 \pm 5$ \\
\hline Brown, 2018 [195] & $\begin{array}{l}3 T, T 1 \text { weighted saturation recovery single-shot GRE, at rest and under adenosine } \\
\text { stress }\end{array}$ & 19:23 & $23(22-29)^{b}$ \\
\hline
\end{tabular}

$n$ number of study participants, GRE gradient echo

a Analysis of a subset of healthy subjects (without hypertension, no use of antihypertensive or other medication for a cardiovascular condition, no diabetes, normal glucose tolerance, no smoking history and normal total cholesterol $(<240 \mathrm{mg} / \mathrm{dl})$ ) of the original cohort

${ }^{\mathrm{b}}$ Median (interquartile range)

Table 69 Reference ranges of normal absolute myocardial blood flow (MBF) at rest and during adenosine stress and perfusion reserve in adults and children

\begin{tabular}{|c|c|c|c|c|}
\hline References & n & $\begin{array}{l}\text { MBF at rest }(\mathrm{ml} / \mathrm{min} / \mathrm{g}) \\
\text { Mean } \pm \mathrm{SD}(\mathrm{LL}-\mathrm{UL})^{\mathrm{b}}\end{array}$ & $\begin{array}{l}\text { MBF during Adenosine stress }(\mathrm{ml} / \mathrm{min} / \mathrm{g}) \\
\text { Mean } \pm \mathrm{SD}(\mathrm{LL}-\mathrm{UL})^{\mathrm{b}}\end{array}$ & $\begin{array}{l}\text { Perfusion reserve }^{a} \\
\text { Mean } \pm S D(L L-U L)^{b}\end{array}$ \\
\hline Wang, 2006 [192] & $\begin{array}{l}99 \\
49 \\
50\end{array}$ & $\begin{array}{l}\text { All: } 1.02 \pm 0.24(0.54-1.5) \\
\text { Men: } 0.96 \pm 0.23(0.5-1.96) \\
\text { Women: } 1.08 \pm 0.23(0.62-2.32)\end{array}$ & $\begin{array}{l}\text { All: } 3.13 \pm 0.80(1.53-6.19) \\
\text { Men: } 2.79 \pm 0.72(1.35-5.49) \\
\text { Women: } 3.46 \pm 0.73(2.02-7.50)\end{array}$ & All: $3.17 \pm 0.87(1.43-4.91)$ \\
\hline Madriago, 2015 [196] c & 20 & $0.94 \pm 0.17(0.6-1.28)$ & $2.34 \pm 0.82(0.7-3.98)$ & $2.63 \pm 0.96(0.71-4.55)$ \\
\hline Brown, 2018 [195] & 42 & $0.65 \pm 0.13(0.39-0.91)$ & $2.71 \pm 0.61(1.49-3.93)$ & $4.24 \pm 0.69(2.86-5.62)$ \\
\hline
\end{tabular}

$n$ number of study participants, $M B F$ myocardial blood flow

${ }^{a}$ Ratio of MBF during stress divided by $M B F$ at rest

${ }^{b}$ Calculated as mean $\pm 2 * S D$

' Data table was made available by senior author to calculate mean and SD

differ substantially. Therefore, in this review we abstained from calculation of weighted mean values and present reference ranges given in the two publications separately (Table 69).

There is a single study presenting reference ranges of myocardial perfusion in children [196] (Table 68). Although the sample size is small $(n=20)$ and children have cardiovascular pathologies (e.g. atrial and ventricular septal defects), data is presented here since a study of myocardial stress perfusion imaging in a larger subset of entirely healthy children seems not feasible (Table 68).

\section{Artificial Intelligence (AI)-based segmentation methods for analysis of cine MRI}

Currently no AI-based normal values have been published in the literature. In recent years however, major improvements have been made in the development of automated CMR segmentation methods based on AI technology using so called Convolutional Neural Networks (CNN). Most published work report methods for automated LV and RV segmentation in Cine CMR [3, 197-203]. CNN based methods have also been presented for automated quantification of atrial dimensions $[2,204]$, myocardial scar tissue from LGE [205, 206], T1 mapping [207], aortic flow [208] and disease classification [209]. Given the potential importance of this topic to the field of CMR, this section summarizes relevant literature in this area and provides a summary of the publicly available CMR data sets relevant to AI segmentation of CMR data.

CNN-based automated image segmentation methods rely on training data, i.e. images with known segmentation result, to derive a neural network with multiple nodes, layers and weighting parameters that can be applied to unseen images to label every pixel in the image. The structure of the neural network varies among publications, but many are based on or are similar to the UNET structure introduced by Ronnenberger et al. in 2015 [210]. The applicability of a CNN implementation is highly dependent on the data that was used to train the weights in the CNN. Ideally, the training data is representative for the data for which the $\mathrm{CNN}$ is to be used. Important considerations include the mix of pathologies, mix of CMR scanner vendors and variation in CMR acquisition parameters in the training set. Many published CCN methods for CMR image segmentation are based on data sets that have been made publicly available in the setting of so called challenges, i.e. competitions 
Table $\mathbf{7 0}$ Publicly available data sets that have been used for training and testing for automated segmentation algorithms of the left and right ventricle

\begin{tabular}{|c|c|c|c|c|}
\hline Data set & Conference/source & $n$ & Segmented structure & Data description \\
\hline MICCAI-2009 [213] & MICCAI 2009 & 45 & Left ventricle & $\begin{array}{l}\text { Single center, single vendor } \\
5 \text { sub-groups: healthy, hypertrophy, heart failure with infarction and } \\
\text { heart failure without infarction } \\
\text { Data hosted on: https://www.cardiacatlas.org/studies/sunnybrook- } \\
\text { cardiac-data/ }\end{array}$ \\
\hline LVSC-2011 [214] & STACOM-2011 & 200 & Left ventricle & $\begin{array}{l}\text { Multi center, multi-vendor } \\
\text { Myocardial infarction }\end{array}$ \\
\hline RVSC-2012 [215] & MICCAI 2012 & 48 & Right ventricle & $\begin{array}{l}\text { Single center } \\
\text { Randomly selected clinical cases }\end{array}$ \\
\hline ACDC-2017 [198] & MICCAI 2017 & 150 & Left ventricle & $\begin{array}{l}\text { Single center, } 2 \text { scanners, } 1 \text { vendor } \\
5 \text { sub-groups (Normal, post-myocardial infarction, dilated cardio- } \\
\text { myopathy, hypertrophic cardiomyopathy, abnormal right ventricle) }\end{array}$ \\
\hline KAGGLE-2015 [216] & $\begin{array}{l}\text { KAGGLE 2015s annual } \\
\text { data science bow }\end{array}$ & 1100 & Left ventricle & $\begin{array}{l}\text { Multi center, multi scanner } \\
\text { Mix of patient and volunteer scans } \\
\text { Only end-diastolic and end-systolic ground truth results provided. } \\
\text { No gold standard segmentations available }\end{array}$ \\
\hline Multiple sources & Cardiac Atlas Project [217] & $>6500$ & Left ventricle & $\begin{array}{l}\text { Multi center, multi-vendor } \\
\text { Asymptomatic subjects } \\
\text { Data acquired with gradient echo cine acquisition } \\
\text { Data hosted on: https://www.cardiacatlas.org/studies/mesa/ }\end{array}$ \\
\hline
\end{tabular}

$n$ number of subjects, MICCAI Medical Image Computing and Computer Assisted Intervention, LVSC Left Ventricle Segmentation Challenge, STACOM Statistical Atlases and Computational Modelling of the Heart, RVSC Right Ventricle Segmentation Challenge, ACDC Automatic Cardiac Diagnosis Challenge

in which participants are invited to develop the best segmentation algorithm for a given type of data [211]. Table 70 lists the most relevant public CMR Cine CMR data sets that have been used for this purpose.

Validation of CNN based segmentation methods is based on comparing the results of automated segmentation with manual results from a trained observer. Commonly used geometrical validation metrics include the Dice overlap, Haussdorf distance and average distance between contours [198]. Additionally, derived quantitative parameters from either automated or manual contours can be compared. As manual analysis is subject to observer bias and variability it can only serve as surrogate gold standard. Some papers report the observer variability of manual analysis in order to assess how the limits of agreement of an automated method compare with the limits of agreement within or between manual observers.

CNN-based image segmentation methods are being introduced in commercially available image analysis software packages. The question arises whether results from such automated methods can be used interchangeably with results from manual image analysis. Although CNN's are designed to replicate the image segmentation performed by an expert observer, it is conceivable that relevant differences may occur, especially in myocardial pathologies which were not well represented in the cohort that was used to train the $\mathrm{CNN}$.

\section{Studies included in this review}

Table 71 lists 11 studies presenting CNN based image segmentation methods for automated analysis of CMR imaging data. Studies are included based on having either used a well described public data set for training and testing, or a dataset of $>300$ subjects selected according to a properly described inclusion protocol. Most published work have used publicly available datasets for training and testing of algorithms for LV [198-202, 204] or RV [198, 200, 202-204] segmentation in short-axis cine CMR. The use of public data sets for algorithm training and validation enables objective comparison of the performance of the methods. Due to the relatively small size of training sets used and the limitation in variation in patient pathology, scanner manufactures, field strength and scanning protocol, it is uncertain how these methods perform on routine clinical CMR data. However, the above studies do convincingly demonstrate the high potential of $\mathrm{CNN}$ based image segmentation.

There are several studies for which AI methods were developed and applied to larger cohorts of subjects. Bai et al. presented a $\mathrm{CNN}$ method that was trained on a large dataset of 4875 subject scans of the UK BioBank cohort [2]. This method provides automated segmentation and quantification of short-axis and long-axis cine CMR for all four heart chambers. It was shown that the method provides excellent segmentation results when applied to cases from the UK Biobank cohort. However, 
Table 71 Recent studies describing fully automated LV or RV segmentation algorithm based on Convolutional Neural Networks (CNN)

\begin{tabular}{|c|c|c|c|}
\hline Author, year & Segmented structure & Data used for training/validation & Validation methods/remarks \\
\hline Tran, 2017 [203] & $L V+R V$ & $\begin{array}{l}\text { LV: MICCAI-2009 }(n=45) \\
\text { RV: RVSC-2012 }(n=45)\end{array}$ & $\begin{array}{l}\text { Validation: auto vs manual } \\
\text { Metrics: DICE, HD, ACD }\end{array}$ \\
\hline Bai, 2018 [2] & $\begin{array}{l}\mathrm{LV}+\mathrm{RV} \\
\text { Including LV, RV, LA, RA from } \\
\text { long-axis cine }\end{array}$ & $\begin{array}{l}4875 \text { subjects } \\
\text { UK Biobank cohort } \\
\text { Multi-center, single vendor }\end{array}$ & $\begin{array}{l}\text { Validation: auto vs manual } \\
\text { Metrics: DICE, HD, APD } \\
\text { LV: EDV, ESV, EF, SV, CO, LV mass } \\
\text { RV: EDV, ESV, EF, CO }\end{array}$ \\
\hline Bernard, 2018 [198] & $L V+R V$ & ACDC-2017 $(n=150)$ & $\begin{array}{l}\text { Nine methods compared } \\
\text { Validation: auto vs manual } \\
\text { Metrics: DICE, HD, CLBR } \\
\text { LV: EDV, ESV, EF, LV mass } \\
\text { RV: EDV, ESV, EF }\end{array}$ \\
\hline Khened, 2018 [200] & $L V+R V$ & $\begin{array}{l}\text { KAGGLE-2015 }(n=1140) \\
\text { ACDC-2017 }(n=150) \\
\text { LVSC-2011 }(n=200)\end{array}$ & $\begin{array}{l}\text { Validation: auto vs manual } \\
\text { Metrics: DICE, CLBR } \\
\text { Patient diagnosis }\end{array}$ \\
\hline Tan, 2018 [201] & LV & $\begin{array}{l}\text { LVSC-2011 }(n=200) \\
\text { KAGGLE-2015 }(n=1140)\end{array}$ & $\begin{array}{l}\text { Validation: auto vs manual } \\
\text { Metrics: DICE, Jl, HD } \\
\text { EDV, ESV }\end{array}$ \\
\hline Tao, 2018 [3] & LV & $\begin{array}{l}\text { Training: } 400 \text { subjects } \\
\text { Testing: } 150 \text { subjects } \\
\text { Multi-center, multi-vendor } \\
\text { Multiple patient categories: MI }(n=322), \text { DCM } \\
(n=168), \operatorname{HCM}(n=23), D C M(n=23), \text { PH }(n=10) \\
\text { other }(n=27) \text {, normal }(n=23)\end{array}$ & $\begin{array}{l}\text { Validation: auto vs manual } \\
\text { Metrics: DICE } \\
\text { EDV, ESV, EF, LV mass }\end{array}$ \\
\hline Vigneault, 2018 [204] & $\mathrm{LV}+\mathrm{RV}$ from multiple views & $\begin{array}{l}53 \text { subjects } \\
\text { HCM }(n=42) \text {, healthy }(n=21) \\
\text { ACDC-2017 }(n=150)\end{array}$ & $\begin{array}{l}\text { Validation: auto vs manual } \\
\text { Metrics: DICE }\end{array}$ \\
\hline Backhaus, 2019 [212] & $L V+R V$ & $\begin{array}{l}\text { Evaluation of SuiteHEART software (Neosoft) } \\
300 \text { randomly selected patients used for validation } \\
\text { Single center } \\
1.5 T \text { and } 3 T \text { data }\end{array}$ & $\begin{array}{l}\text { Validation: auto vs manual } \\
\text { LV: EDV, ESV, SV, EF, LV mass } \\
\text { RV: EDV, ESV, EF }\end{array}$ \\
\hline Bhuva, 2019 [197] & LV & $\begin{array}{l}\text { Training data: } 599 \text { subjects } \\
\text { Test data } 110 \text { patients, } 5 \text { disease categories: myo- } \\
\text { cardial infarction }(n=32), \mathrm{LVH}(\mathrm{n}=17) \text {, cardiomyo- } \\
\text { pathy }(\mathrm{n}=17) \text {, other pathology }(\mathrm{n}=14) \text {, healthy } \\
\text { volunteers }(\mathrm{n}=30) \\
\text { Multi-center, multi-vendor, } 1.5 T+3 T \\
\text { Scan-rescan data } \\
\text { Data availability: } \\
\text { https://www.thevolumesresource.com }\end{array}$ & $\begin{array}{l}\text { Validation based on comparing scan-rescan } \\
\text { reproducibility of automated vs manual } \\
\text { analysis } \\
\text { EDV, ESV, SV, EF, LV mass } \\
\text { Detectable change in EF }\end{array}$ \\
\hline Curiale, 2019 [199] & LV & $\begin{array}{l}\text { MICCAI-2009 }(n=45) \\
\text { Cardiac Atlas Project }(n=95)\end{array}$ & $\begin{array}{l}\text { Validation: auto vs manual } \\
\text { Metrics: DICE } \\
\text { EDV, ESV, EF, LV mass }\end{array}$ \\
\hline Tong, 2019 [202] & $L V+R V$ & ACDC-2017 $(n=150)$ & $\begin{array}{l}\text { Validation: auto vs manual } \\
\text { Metrics: Dice, HD } \\
\text { LV: EDV, ESV, EF, LV mass } \\
\text { RV: EDV, ESV, EF }\end{array}$ \\
\hline
\end{tabular}

Recent studies (> 2017) describing fully automated LV or RV segmentation algorithm based on Convolutional Neural Networks (CNN), which were validated either on publicly available data sets, or using lager (> 300 subjects) single-center or multi-center clinical patient cohorts. Segmentation is performed from short-axis cine MR, except stated otherwise.

$L V$ left ventricle, $R V$ right ventricle, $L A$ left atrium, $R A$ right atrium, $M I$ myocardial infarction, $D C M$ dilated cardiomyopathy, $H C M$ hypertrophic cardiomyopathy, $P H$ pulmonary hypertension, DICE dice overlap metric, HD Hausdorff distance, Jl Jaccard index, CLBR challenge leader board ranking, $A C D$ average contour distance, MICCAI Medical Image Computing and Computer Assisted Intervention, LVSC Left Ventricle Segmentation Challenge, ACDC Automatic Cardiac Diagnosis Challenge.

for application in clinical patients, the method demonstrated sub-optimal performance. Retraining the network by including additional cases of a clinical cohort did result in better results in patient data.

In the study of Tao et al. multi-center, multi-vendor, multi-pathology data was used to train and test vendor specific CNNs and a mixed-vendor CNN [3]. The authors showed that the $\mathrm{CNN}$ trained using a mix of data from all centers, vendors and pathologies had the highest overall performance. This indicates that it is feasible to use the same optimally trained $\mathrm{CNN}$ across multiple centers, vendors and patient pathologies. 
A retrospective clinical validation of a commercial image analysis software tool was presented by Backhaus et al. [212]. In a randomly selected cohort of 300 CMR examination LV and RV parameters were automatically derived using a commercial software tool (SuiteHEART, NeoSoft, Pewaukee, Wisconsin, USA) incorporating $\mathrm{CNN}$ based image segmentation. The agreement between manual and automated LV parameter assessment was good (Bias in LV-EF: $-2.5 \% \pm 5.9 \%$ ), while for RV assessment the agreement was lower (Bias in RV EF: $5.8 \% \pm 9.6 \%)$. As expected, the agreement between manual and automated analysis was lowest in cases of poor image quality and in patients with abnormal cardiac anatomy.

Bhuva et al. used another approach to assess the performance of $\mathrm{CNN}$ based image segmentation as compared to manual analysis [197]. In their study a CNN LV segmentation method was trained on 599 subjects and tested on scan-rescan data of 110 patients with multiple pathologies. It was shown that automated image segmentation yielded similar scan-rescan reproducibility as manual image analysis, which suggests that automated segmentation is a viable alternative to manual analysis in a clinical setting.

\section{Conclusions}

CMR enables quantification of various functional and morphological parameters of the cardiovascular system. Advantages of a quantitative evaluation are a better differentiation between pathology and normal conditions, grading of pathologies, monitoring changes under therapy, and evaluating prognosis and the possibility of comparing different groups of patients and normal subjects.

Hence, here we present an updated and expanded version of the "normal value CMR review". This review has provided reference values and factors affecting these parameters on current CMR techniques and sequences. Due to continuing publications in the field and new techniques transferred from research tools into clinical practice existing reference ranges need to be updated and values for new techniques integrated.

\footnotetext{
Abbreviations

AA: Ascending aorta; ACDC: Automatic Cardiac Diagnosis Challenge; AHA: American Heart Association; Al: Artificial intelligence; ARVC: Arrhythmogenic right ventricular cardiomyopathy; AVPD: Atrioventricular plane descent; BMI: Body mass index; BSA: Body surface area; bSSFP: Balanced steady state free precession; CE: Contrast enhanced; Ch: Chamber; Cl: Cardiac index; CLBR: Challenge leader board ranking; CO: Cardiac output; CMR: Cardiovascular magnetic resonance; CMRA: Cardiovascular magnetic resonance angiography; CNN: Convolutional neural network; DENSE: Displacement encoding with stimulated echoes; DCM: Dilated cardiomyopathy; DICE: Dice overlap metric; ECG: Electrocardiogram; ECV: Extracellular volume; EDV: End-diastolic volume; EF: Ejection fraction; ESV: End-systolic volume; FD: Fractal dimension; FT: Feature-tracking; FGRE: Fast gradient echo; GRASE: Gradient and spin echo;
}

GRE: Gradient echo; HARP: Harmonic phase analysis; HD: Hausdorff distance; HCM: Hypertrophic cardiomyopathy; IVS: Interventricular septum; Jl: Jaccard index; LA: Left atrial/left atrial; LL: Lower limit (of normal); LV: Left ventricle/ left ventricular; LVEF: Left ventricular ejection fraction; LVM: Left ventricular mass; LVOT: Left ventricular outflow tract; LVSC: Left Ventricle Segmentation Challenge; LMS: Lambda Mu Sigma; LPA: Left pulmonary artery; MBF: Myocardial blood flow; Mean;: Pooled weighted mean; MESA: Multi-Ethnic Study of Atherosclerosis; MICCAl: Medical Image Computing and Computer Assisted Intervention; MOLLI: Modified Look-Locker inversion recovery; MPA: Main pulmonary artery; MRI: Magnetic resonance imaging; MRA: MR angiography; NC/C: Non-compacted/compacted (left ventricular myocardium); PFR: Peak filling rate; PC: Phase contrast; RA: Right atrium/right atrial; RF: Radiofrequency; RPA: Right pulmonary artery; RPP: Rate-pressure product; RV: Right ventricle/ right ventricular; RVSC: Right Ventricle Segmentation Challenge; SASHA: Saturation Recovery Single-sHot Acquisition; SENC: Strain-encoding; SD: Standard deviation; SD : Pooled weighted standard deviation; ShMOLLI: Shortened modified Look-Locker inversion recovery; SinMod: Sine wave modeling; SPAMM: Spatial modulation of magnetization; STACOM: Statistical Atlases and Computational Modelling of the Heart; SV: Stroke volume; T: Tesla; T1: T1-relaxation time; T2: T2-relaxation time; TM: Total (left ventricular myocardial) mass; TSE: Turbo-spin echo; TTE: Transthoracic echocardiography; UL: Upper limit (of normal); $V_{\text {enc }}$ : Flow encoding velocity; VISTA: Volume isotropic surbo spin echo; yrs: Years.

\section{Acknowledgements}

The project was supported by the Clinical and Translational Science Award (CTSA) program, through the NIH National Center for Advancing Translational Sciences (NCATS), grant UL1TR002373. The content is solely the responsibility of the authors and does not necessarily represent the official views of the $\mathrm{NIH}$.

\section{Authors' contributions}

Concept and design of study: NKB and DAB. Literature search: all authors. Manuscript drafting: all authors. Data analysis: SH. Manuscript revision: NKB and DAB. All authors read and approved the final manuscript.

\section{Funding}

None.

Availability of data and materials

Not applicable.

Ethics approval and consent to participate

Not applicable.

\section{Consent for publication}

Not applicable.

\section{Competing interests}

CJF: Research support by GE healthcare. MS: Research support by Siemens healthcare. SDT: Boehringer Ingelheim speaker bureau.

\section{Author details}

${ }^{1}$ Department of Radiology, Kantonsspital Graubuenden, Loestrasse 170, 7000 Chur, Switzerland. ${ }^{2}$ Institute for Diagnostic, Interventional and Pediatric Radiology (DIPR), Bern University Hospital, University of Bern, Freiburgstrasse 10, 3010 InselspitalBern, Switzerland. ${ }^{3}$ Department of Biostatistics and Medical Informatics, University of Wisconsin, 610 Walnut St, Madison, WI 53726, USA. ${ }^{4}$ Department of Radiology, Johns Hopkins University, 600 N Wolfe Street, Baltimore, MD 21287, USA. ${ }^{5}$ MRC Unit of Lifelong Health and Ageing At UCL, 5-19 Torrington Place, Fitzrovia, London WC1E 7HB, UK. ${ }^{6}$ Inherited Heart Muscle Conditions Clinic, Royal Free Hospital NHS Foundation Trust, Hampstead, London NW3 2QG, UK. ${ }^{7}$ Department of Radiology, University of Wisconsin School of Medicine and Public Health, 600 Highland Avenue, Madison, WI 53792, USA. ${ }^{8}$ Department of Radiology, Brigham and Women's Hospital, 75 Francis Street, Boston, MA 02115, USA. ${ }^{9}$ Cardiovascular Division, University of Virginia Health System, 1215 Lee Street, Charlottesville, VA 22908, USA. ${ }^{10}$ Department of Radiology, National Jewish Health, 1400 Jackson St, Denver, CO 80206, USA.

${ }^{11}$ Division of Paediatric Cardiology, University Children's Hospital Zurich, Steinwiesstrasse 75, 8032 Zurich, Switzerland. ${ }^{12}$ Department of Radiology, Leiden University Medical Center, Albinusdreef 2, 2333ZA Leiden, The Netherlands. 
Received: 9 January 2020 Accepted: 26 October 2020

Published online: 14 December 2020

\section{References}

1. Kawel-Boehm N, Maceira A, Valsangiacomo-Buechel ER, Vogel-Claussen J, Turkbey EB, Williams R, Plein S, Tee M, Eng J, Bluemke DA. Normal values for cardiovascular magnetic resonance in adults and children. J Cardiovasc Magn Reson. 2015;17:29.

2. Bai W, Sinclair M, Tarroni G, Oktay O, Rajchl M, Vaillant G, Lee AM, Aung $\mathrm{N}$, Lukaschuk E, Sanghvi MM, et al. Automated cardiovascular magnetic resonance image analysis with fully convolutional networks. J Cardiovasc Magn Reson. 2018;20:65.

3. Tao Q, Yan W, Wang Y, Paiman EHM, Shamonin DP, Garg P, Plein S, Huang L, Xia L, Sramko M, et al. Deep Learning-based Method for Fully Automatic Quantification of Left Ventricle Function from Cine MR Images: A Multivendor Multicenter Study. Radiology. 2019;290:81-8.

4. Horowitz GL. Estimating reference intervals. Am J Clin Pathol. 2010;133:175-7.

5. SCMR: Consensus/Position statements, [https://scmr.org/general/ custom.asp?page = quidelines], Accessed 15 Dec 2019

6. Chuang ML, Gona P, Hautvast GL, Salton CJ, Blease SJ, Yeon SB, Breeuwer M, O'Donnell CJ, Manning WJ. Correlation of trabeculae and papillary muscles with clinical and cardiac characteristics and impact on CMR measures of LV anatomy and function. JACC Cardiovasc Imaging. 2012;5:1115-23.

7. Riffel JH, Schmucker K, Andre F, Ochs M, Hirschberg K, Schaub E, Fritz T, Mueller-Hennessen M, Giannitsis E, Katus HA, Friedrich MG. Cardiovascular magnetic resonance of cardiac morphology and function: impact of different strategies of contour drawing and indexing. Clin Res Cardiol. 2019;108:411-29.

8. Vogel-Claussen J, Finn JP, Gomes AS, Hundley GW, Jerosch-Herold M, Pearson G, Sinha S, Lima JA, Bluemke DA. Left ventricular papillary muscle mass: relationship to left ventricular mass and volumes by magnetic resonance imaging. J Comput Assist Tomogr. 2006;30:426-32.

9. Schulz-Menger J, Bluemke DA, Bremerich J, Flamm SD, Fogel MA, Friedrich MG, Kim RJ, von Knobelsdorff-Brenkenhoff F, Kramer CM, Pennell DJ, et al. Standardized image interpretation and post-processing in cardiovascular magnetic resonance-2020 update: Society for Cardiovascular Magnetic Resonance (SCMR): Board of Trustees Task Force on Standardized Post-Processing. J Cardiovasc Magn Reson. 2020;22:19.

10. Maceira AM, Prasad SK, Khan M, Pennell DJ. Normalized left ventricular systolic and diastolic function by steady state free precession cardiovascular magnetic resonance. J Cardiovasc Magn Reson. 2006a;8:417-26.

11. Le TT, Tan RS, De Deyn M, Goh EP, Han Y, Leong BR, Cook SA, Chin CW. Cardiovascular magnetic resonance reference ranges for the heart and aorta in Chinese at 3T. J Cardiovasc Magn Reson. 2016;18:21.

12. Bentatou Z, Finas M, Habert P, Kober F, Guye M, Bricq S, Lalande A, Frandon J, Dacher JN, Dubourg B, et al. Distribution of left ventricular trabeculation across age and gender in 140 healthy Caucasian subjects on MR imaging. Diagn Interv Imaging. 2018;99:689-98.

13. Bulow R, Ittermann T, Dorr M, Poesch A, Langner S, Volzke H, Hosten $N_{\text {, }}$ Dewey M. Reference ranges of left ventricular structure and function assessed by contrast-enhanced cardiac MR and changes related to ageing and hypertension in a population-based study. Eur Radiol. 2018:28:3996-4005

14. Le Ven F, Bibeau K, De Larochelliere E, Tizon-Marcos H, DeneaultBissonnette S, Pibarot P, Deschepper CF, Larose E. Cardiac morphology and function reference values derived from a large subset of healthy young Caucasian adults by magnetic resonance imaging. Eur Heart J Cardiovasc Imaging. 2016;17:981-90.

15. Lei X, Liu H, Han Y, Cheng W, Sun J, Luo Y, Yang D, Dong Y, Chung Y, Chen Y. Reference values of cardiac ventricular structure and function by steady-state free-procession MRI at 3.0T in healthy adult chinese volunteers. J Magn Reson Imaging. 2017;45:1684-92.

16. Petersen SE, Aung N, Sanghvi MM, Zemrak F, Fung K, Paiva JM, Francis JM, Khanji MY, Lukaschuk E, Lee AM, et al. Reference ranges for cardiac structure and function using cardiovascular magnetic resonance (CMR) in Caucasians from the UK Biobank population cohort. J Cardiovasc Magn Reson. 2017;19:18.
17. Reiter G, Reiter U, Rienmuller R, Gagarina N, Ryabikin A. On the value of geometry-based models for left ventricular volumetry in magnetic resonance imaging and electron beam tomography: a Bland-Altman analysis. Eur J Radiol. 2004:52:110-8.

18. Aquaro GD, Camastra G, Monti L, Lombardi M, Pepe A, Castelletti S, Maestrini V, Todiere G, Masci P, di Giovine G, et al. Reference values of cardiac volumes, dimensions, and new functional parameters by MR: A multicenter, multivendor study. J Magn Reson Imaging. 2017:45:1055-67.

19. Gandy SJ, Lambert M, Belch J, Cavin I, Crowe E, Littleford R, MacFarlane JA, Matthew SZ, Martin P, Nicholas RS, et al. 3T MRI investigation of cardiac left ventricular structure and function in a UK population: The tayside screening for the prevention of cardiac events (TASCFORCE) study. J Magn Reson Imaging. 2016;44:1 186-96.

20. Maroules CD, McColl R, Khera A, Peshock RM. Interstudy reproducibility of SSFP cine magnetic resonance: impact of magnetic field strength and parallel imaging. J Magn Reson Imaging. 2008;27:1139-45.

21. Natori S, Lai S, Finn JP, Gomes AS, Hundley WG, Jerosch-Herold M, Pearson G, Sinha S, Arai A, Lima JA, Bluemke DA. Cardiovascular function in multi-ethnic study of atherosclerosis: normal values by age, sex, and ethnicity. AJR Am J Roentgenol. 2006;186:S357-365.

22. Hudsmith LE, Petersen SE, Francis JM, Robson MD, Neubauer S. Normal human left and right ventricular and left atrial dimensions using steady state free precession magnetic resonance imaging. J Cardiovasc Magn Reson. 2005;7:775-82.

23. Chang SA, Choe YH, Jang SY, Kim SM, Lee SC, Oh JK. Assessment of left and right ventricular parameters in healthy Korean volunteers using cardiac magnetic resonance imaging: change in ventricular volume and function based on age, gender and body surface area. Int J Cardiovasc Imaging. 2012;28(Suppl 2):141-7.

24. Macedo R, Fernandes JL, Andrade SS, Rochitte CE, Lima KC, Maciel AC, Maciel FC, Alves GS, Coelho OR, Diniz RV. Morphological and functional measurements of the heart obtained by magnetic resonance imaging in Brazilians. Arq Bras Cardiol. 2013;101:68-77.

25. Yeon SB, Salton CJ, Gona P, Chuang ML, Blease SJ, Han Y, Tsao CW, Danias PG, Levy D, O'Donnell CJ, Manning WJ. Impact of age, sex, and indexation method on MR left ventricular reference values in the Framingham Heart Study offspring cohort. J Magn Reson Imaging. 2015;41:1038-45.

26. Liu B, Dardeer AM, Moody WE, Hayer MK, Baig S, Price AM, Leyva F, Edwards NC, Steeds RP. Reference ranges for three-dimensional feature tracking cardiac magnetic resonance: comparison with two-dimensional methodology and relevance of age and gender. Int J Cardiovasc Imaging. 2018;34:761-75.

27. Sievers B, Kirchberg S, Bakan A, Franken U, Trappe HJ. Impact of papillary muscles in ventricular volume and ejection fraction assessment by cardiovascular magnetic resonance. J Cardiovasc Magn Reson. 2004;6:9-16.

28. Winter MM, Bernink FJ, Groenink M, Bouma BJ, van Dijk AP, Helbing WA, Tijssen JG, Mulder BJ. Evaluating the systemic right ventricle by CMR: the importance of consistent and reproducible delineation of the cavity. J Cardiovasc Magn Reson. 2008;10:40.

29. Maceira AM, Prasad SK, Khan M, Pennell DJ. Reference right ventricular systolic and diastolic function normalized to age, gender and body surface area from steady-state free precession cardiovascular magnetic resonance. Eur Heart J. 2006b;27:2879-88.

30. Nacif MS, Barranhas AD, Turkbey E, Marchiori E, Kawel N, Mello RA, Falcao RO, Oliveira AC Jr, Rochitte CE. Left atrial volume quantification using cardiac MRI in atrial fibrillation: comparison of the Simpson's method with biplane area-length, ellipse, and three-dimensional methods. Diagn Interv Radiol. 2013;19:213-20.

31. Maceira AM, Cosin-Sales J, Roughton M, Prasad SK, Pennell DJ. Reference left atrial dimensions and volumes by steady state free precession cardiovascular magnetic resonance. J Cardiovasc Magn Reson. 2010;12:65.

32. Funk S, Kermer J, Doganguezel S, Schwenke C, von Knobelsdorff-Brenkenhoff F, Schulz-Menger J. Quantification of the left atrium applying cardiovascular magnetic resonance in clinical routine. Scand Cardiovasc J. 2018;52:85-92. 
33. Li W, Wan K, Han Y, Liu H, Cheng W, Sun J, Luo Y, Yang D, Chung YC, Chen $Y$. Reference value of left and right atrial size and phasic function by SSFP CMR at 3.0 T in healthy Chinese adults. Sci Rep. 2017;7:3196.

34. Zemrak F, Ambale-Venkatesh B, Captur G, Chrispin J, Chamera E, Habibi M, Nazarian S, Mohiddin SA, Moon JC, Petersen SE, et al. Left Atrial Structure in Relationship to Age, Sex, Ethnicity, and Cardiovascular Risk Factors: MESA (Multi-Ethnic Study of Atherosclerosis). Circ Cardiovasc Imaging. 2017;10:9.

35. Sievers B, Kirchberg S, Franken U, Bakan A, Addo M, John-Puthenveettil $B$, Trappe HJ. Determination of normal gender-specific left atrial dimensions by cardiovascular magnetic resonance imaging. J Cardiovasc Magn Reson. 2005;7:677-83.

36. Rohner A, Brinkert M, Kawel N, Buechel RR, Leibundgut G, Grize L, Kuhne M, Bremerich J, Kaufmann BA, Zellweger MJ, et al. Functional assessment of the left atrium by real-time three-dimensional echocardiography using a novel dedicated analysis tool: initial validation studies in comparison with computed tomography. Eur J Echocardiogr. 2011;12:497-505.

37. Maceira AM, Cosin-Sales J, Roughton M, Prasad SK, Pennell DJ. Reference right atrial dimensions and volume estimation by steady state free precession cardiovascular magnetic resonance. J Cardiovasc Magn Reson. 2013;15:29

38. Maceira AM, Cosin-Sales J, Prasad SK, Pennell DJ. Characterization of left and right atrial function in healthy volunteers by cardiovascular magnetic resonance. J Cardiovasc Magn Reson. 2016;18:64.

39. Buechel EV, Kaiser T, Jackson C, Schmitz A, Kellenberger CJ. Normal right- and left ventricular volumes and myocardial mass in children measured by steady state free precession cardiovascular magnetic resonance. J Cardiovasc Magn Reson. 2009;11:19.

40. Robbers-Visser D, Boersma E, Helbing WA. Normal biventricular function, volumes, and mass in children aged 8 to 17 years. J Magn Reson Imaging. 2009;29:552-9.

41. Sarikouch S, Peters B, Gutberlet M, Leismann B, Kelter-Kloepping A, Koerperich H, Kuehne T, Beerbaum P. Sex-specific pediatric percentiles for ventricular size and mass as reference values for cardiac MRI: assessment by steady-state free-precession and phase-contrast MRI flow. Circ Cardiovasc Imaging. 2010;3:65-76.

42. Dewey FE, Rosenthal D, Murphy DJ Jr, Froelicher VF, Ashley EA. Does size matter? Clinical applications of scaling cardiac size and function for body size. Circulation. 2008;117:2279-87.

43. Sluysmans T, Colan SD. Theoretical and empirical derivation of cardiovascular allometric relationships in children. J Appl Physiol. 1985;2005(99):445-57.

44. Cole TJ. The LMS method for constructing normalized growth standards. Eur J Clin Nutr. 1990;44:45-60.

45. van der Ven JPG, Sadighy Z, Valsangiacomo Buechel ER, Sarikouch S, Robbers-Visser D, Kellenberger CJ, Kaiser T, Beerbaum P, Boersma E, Helbing WA. Multicentre reference values for cardiac magnetic resonance imaging derived ventricular size and function for children aged 0-18 years. Eur Heart J Cardiovasc Imaging. 2019;89:109.

46. Schulz-Menger J, Bluemke DA, Bremerich J, Flamm SD, Fogel MA, Friedrich MG, Kim RJ, von Knobelsdorff-Brenkenhoff F, Kramer CM, Pennell $D J$, et al. Standardized image interpretation and post processing in cardiovascular magnetic resonance: Society for Cardiovascular Magnetic Resonance (SCMR) board of trustees task force on standardized post processing. J Cardiovasc Magn Reson. 2013;15:35.

47. Sarikouch S, Koerperich H, Boethig D, Peters B, Lotz J, Gutberlet M, Beerbaum P, Kuehne T. Reference values for atrial size and function in children and young adults by cardiac MR: a study of the German competence network congenital heart defects. J Magn Reson Imaging. 2011;33:1028-39.

48. Mitchell JH, Haskell W, Snell P, Van Camp SP. Task Force 8: classification of sports. J Am Coll Cardiol. 2005;45:1364-7.

49. D'Ascenzi F, Anselmi F, Piu P, Fiorentini C, Carbone SF, Volterrani L, Focardi M, Bonifazi M, Mondillo S. Cardiac Magnetic Resonance Normal Reference Values of Biventricular Size and Function in Male Athlete's Heart. JACC Cardiovasc Imaging. 2019;12:1755-65.

50. Prakken NH, Velthuis BK, Teske AJ, Mosterd A, Mali WP, Cramer MJ. Cardiac MRI reference values for athletes and nonathletes corrected for body surface area, training hours/week and sex. Eur J Cardiovasc Prev Rehabil. 2010;17:198-203.
51. Luijkx T, Velthuis BK, Prakken NH, Cox MG, Bots ML, Mali WP, Hauer RN, Cramer MJ. Impact of revised Task Force Criteria: distinguishing the athlete's heart from ARVC/D using cardiac magnetic resonance imaging. Eur J Prev Cardiol. 2012;19:885-91.

52. Tahir E, Starekova J, Muellerleile K, von Stritzky A, Munch J, Avanesov M, Weinrich JM, Stehning C, Bohnen S, Radunski UK, et al. Myocardial Fibrosis in Competitive Triathletes Detected by Contrast-Enhanced CMR Correlates With Exercise-Induced Hypertension and Competition History. JACC Cardiovasc Imaging. 2018;11:1260-70.

53. Alfakih K, Plein S, Thiele H, Jones T, Ridgway JP, Sivananthan MU. Normal human left and right ventricular dimensions for MRI as assessed by turbo gradient echo and steady-state free precession imaging sequences. J Magn Reson Imaging. 2003;17:323-9.

54. Malayeri AA, Johnson WC, Macedo R, Bathon J, Lima JA, Bluemke DA. Cardiac cine MRI: Quantification of the relationship between fast gradient echo and steady-state free precession for determination of myocardial mass and volumes. J Magn Reson Imaging. 2008;28:60-6.

55. Kawel N, Turkbey EB, Carr JJ, Eng J, Gomes AS, Hundley WG, Johnson C, Masri SC, Prince MR, van der Geest RJ, et al. Normal left ventricular myocardial thickness for middle-aged and older subjects with steady-state free precession cardiac magnetic resonance: the multi-ethnic study of atherosclerosis. Circ Cardiovasc Imaging. 2012;5:500-8.

56. Dawson DK, Maceira AM, Raj VJ, Graham C, Pennell DJ, Kilner PJ. Regional thicknesses and thickening of compacted and trabeculated myocardial layers of the normal left ventricle studied by cardiovascular magnetic resonance. Circ Cardiovasc Imaging. 2011;4:139-46.

57. Captur G, Karperien AL, Li C, Zemrak F, Tobon-Gomez C, Gao X, Bluemke DA, Elliott PM, Petersen SE, Moon JC. Fractal frontiers in cardiovascular magnetic resonance: towards clinical implementation. J Cardiovasc Magn Reson. 2015;17:80.

58. Amzulescu MS, Rousseau MF, Ahn SA, Boileau L. Prognostic impact of hypertrabeculation and noncompaction phenotype in dilated cardiomyopathy: a CMR study. JACC Cardiovasc Imaging. 2015;8:934-46.

59. Captur G, Muthurangu V, Cook C, Flett AS, Wilson R, Barison A, Sado DM, Anderson S, McKenna WJ, Mohun TJ, et al. Quantification of left ventricular trabeculae using fractal analysis. J Cardiovasc Magn Reson. 2013;15:36.

60. Captur G, Lopes LR, Patel V, Li C, Bassett P, Syrris P, Sado DM, Maestrini $\checkmark$, Mohun TJ, McKenna WJ, et al. Abnormal cardiac formation in hypertrophic cardiomyopathy: fractal analysis of trabeculae and preclinical gene expression. Circ Cardiovasc Genet. 2014;7:241-8.

61. Kawel N, Nacif M, Arai AE, Gomes AS, Hundley WG, Johnson WC, Prince MR, Stacey RB, Lima JA, Bluemke DA. Trabeculated (noncompacted) and compact myocardium in adults: the multi-ethnic study of atherosclerosis. Circ Cardiovasc Imaging. 2012;5:357-66.

62. Captur G, Zemrak F, Muthurangu V, Petersen SE, Li C, Bassett P, KawelBoehm N, McKenna WJ, Elliott PM, Lima JA, et al. Fractal analysis of myocardial trabeculations in 2547 study participants: multi-ethnic study of atherosclerosis. Radiology. 2015;277:707-15.

63. LuijkX T, Cramer MJ, Zaidi A, Rienks R, Senden PJ, Sharma S, van Hellemondt FJ, Buckens CF, Mali WP, Velthuis BK. Ethnic differences in ventricular hypertrabeculation on cardiac MRI in elite football players. Neth Heart J. 2012;20:389-95.

64. Andre F, Burger A, Lossnitzer D, Buss SJ, Abdel-Aty H, Gianntisis E, Steen $\mathrm{H}$, Katus HA. Reference values for left and right ventricular trabeculation and non-compacted myocardium. Int J Cardiol. 2015;185:240-7.

65. Tizon-Marcos H, de la Paz RM, Pibarot P, Bertrand O, Bibeau K, Le Ven F, Sinha S, Engert J, Bedard E, Pasian S, et al. Characteristics of trabeculated myocardium burden in young and apparently healthy adults. Am J Cardiol. 2014;114:1094-9.

66. Cai J, Bryant JA, Le TT, Su B, de Marvao A, O'Regan DP, Cook SA, Chin CW. Fractal analysis of left ventricular trabeculations is associated with impaired myocardial deformation in healthy Chinese. J Cardiovasc Magn Reson. 2017;19:102.

67. Sondergaard L, Stahlberg F, Thomsen C, Spraggins TA, Gymoese E, Malmgren L, Muller E, Henriksen O. Comparison between retrospective gating and ECG triggering in magnetic resonance velocity mapping. Magn Reson Imaging. 1993;1 1:533-7.

68. Allen BD, Barker AJ, Carr JC, Silverberg RA, MarkI M. Time-resolved three-dimensional phase contrast MRI evaluation of bicuspid aortic 
valve and coarctation of the aorta. Eur Heart J Cardiovasc Imaging. 2013;14:399.

69. Kupfahl C, Honold M, Meinhardt G, Vogelsberg H, Wagner A, Mahrholdt $\mathrm{H}$, Sechtem U. Evaluation of aortic stenosis by cardiovascular magnetic resonance imaging: comparison with established routine clinical techniques. Heart. 2004;90:893-901.

70. Lotz J, Meier C, Leppert A, Galanski M. Cardiovascular flow measurement with phase-contrast MR imaging: basic facts and implementation. Radiographics. 2002;22:651-71.

71. Srichai MB, Lim RP, Wong S, Lee VS. Cardiovascular applications of phase-contrast MRI. AJR Am J Roentgenol. 2009;192:662-75.

72. Caruthers SD, Lin SJ, Brown P, Watkins MP, Williams TA, Lehr KA, Wickline SA. Practical value of cardiac magnetic resonance imaging for clinical quantification of aortic valve stenosis: comparison with echocardiography. Circulation. 2003;108:2236-43.

73. Kilner PJ, Manzara CC, Mohiaddin RH, Pennell DJ, Sutton MG, Firmin DN, Underwood SR, Longmore DB. Magnetic resonance jet velocity mapping in mitral and aortic valve stenosis. Circulation. 1993:87:1239-48.

74. Myerson SG. Heart valve disease: investigation by cardiovascular magnetic resonance. J Cardiovasc Magn Reson. 2012:14:7.

75. Rathi VK, Doyle M, Yamrozik J, Williams RB, Caruppannan K, Truman C, Vido D, Biederman RW. Routine evaluation of left ventricular diastolic function by cardiovascular magnetic resonance: a practical approach. J Cardiovasc Magn Reson. 2008;10:36

76. Callaghan FM, Bannon P, Barin E, Celemajer D, Jeremy R, Figtree G, Grieve SM. Age-related changes of shape and flow dynamics in healthy adult aortas: a 4D flow MRI study. J Magn Reson Imaging. 2019:49:90-100

77. Garcia J, van der Palen RLF, Bollache E, Jarvis K, Rose MJ, Barker AJ Collins JD, Carr JC, Robinson J, Rigsby CK, Markl M. Distribution of blood flow velocity in the normal aorta: effect of age and gender. J Magn Reson Imaging. 2018:47:487-98.

78. Nishimura RA, Otto CM, Bonow RO, Carabello BA, Erwin JP 3rd, Fleisher LA, Jneid H, Mack MJ, McLeod CJ, O'Gara PT, et al. 2017 AHA/ACC Focused Update of the 2014 AHA/ACC Guideline for the Management of Patients With Valvular Heart Disease: A Report of the American College of Cardiology/American Heart Association Task Force on Clinical Practice Guidelines. J Am Coll Cardiol. 2017:70:252-89.

79. Nishimura RA, Otto CM, Bonow RO, Carabello BA, Erwin JP 3rd, Guyton RA, O'Gara PT, Ruiz CE, Skubas NJ, Sorajja P, et al. 2014 AHA/ACC guideline for the management of patients with valvular heart disease: executive summary: a report of the American College of Cardiology/ American Heart Association Task Force on Practice Guidelines. J Am Coll Cardiol. 2014;63:2438-88.

80. Caudron J, Fares J, Bauer F, Dacher JN. Evaluation of left ventricular diastolic function with cardiac MR imaging. Radiographics. 2011:31:239-59.

81. Kawel N, Jhooti P, Dashti D, Haas T, Winter L, Zellweger MJ, Buser PT, Keegan J, Scheffler K, Bremerich J. MR-imaging of the thoracic aorta: 3D-ECG- and respiratory-gated bSSFP imaging using the CLAWS algorithm versus contrast-enhanced 3D-MRA. Eur J Radiol. 2012;81:239-43.

82. Potthast S, Mitsumori L, Stanescu LA, Richardson ML, Branch K, Dubinsky TJ, Maki JH. Measuring aortic diameter with different MR techniques: comparison of three-dimensional (3D) navigated steady-state free-precession (SSFP), 3D contrast-enhanced magnetic resonance angiography (CE-MRA), 2D T2 black blood, and 2D cine SSFP. J Magn Reson Imaging. 2010;31:177-84

83. Turkbey EB, Jain A, Johnson C, Redheuil A, Arai AE, Gomes AS, Carr 」, Hundley WG, Teixido-Tura G, Eng J, et al. Determinants and normal values of ascending aortic diameter by age, gender, and race/ethnicity in the Multi-Ethnic Study of Atherosclerosis (MESA). J Magn Reson Imaging. 2014;39:360-8.

84. Eikendal AL, Bots ML, Haaring C, Saam T, van der Geest RJ, Westenberg $J$ J, den Ruijter HM, Hoefer IE, Leiner T. Reference values for cardiac and aortic magnetic resonance imaging in healthy young caucasian adults. PLOS ONE. 2016;11:e0164480.

85. Burman ED, Keegan J, Kilner PJ. Aortic root measurement by cardiovascular magnetic resonance: specification of planes and lines of measurement and corresponding normal values. Circ Cardiovasc Imaging. 2008;1:104-13.

86. Davis AE, Lewandowski AJ, Holloway CJ, Ntusi NA, Banerjee R, Nethononda R, Pitcher A, Francis JM, Myerson SG, Leeson P, et al. Observational study of regional aortic size referenced to body size: production of a cardiovascular magnetic resonance nomogram. J Cardiovasc Magn Reson. 2014:16:9.

87. Lederle FA, Johnson GR, Wilson SE, Chute EP, Littooy FN, Bandyk D, Krupski WC, Barone GW, Acher CW, Ballard DJ. Prevalence and associations of abdominal aortic aneurysm detected through screening. Aneurysm Detection and Management (ADAM) Veterans Affairs Cooperative Study Group. Ann Intern Med. 1997;126:441-9.

88. Redheuil A, Yu WC, Wu CO, Mousseaux E, de Cesare A, Yan R, Kachenoura N, Bluemke D, Lima JA. Reduced ascending aortic strain and distensibility: earliest manifestations of vascular aging in humans. Hypertension. 2010;55:319-26.

89. Redheuil A, Yu WC, Mousseaux E, Harouni AA, Kachenoura N, Wu CO, Bluemke D, Lima JA. Age-related changes in aortic arch geometry: relationship with proximal aortic function and left ventricular mass and remodeling. J Am Coll Cardiol. 2011;58:1262-70.

90. Sugawara J, Hayashi K, Yokoi T, Tanaka H. Age-associated elongation of the ascending aorta in adults. JACC Cardiovasc Imaging. 2008;1:739-48.

91. Kaiser T, Kellenberger CJ, Albisetti M, Bergstrasser E, Valsangiacomo Buechel ER. Normal values for aortic diameters in children and adolescents-assessment in vivo by contrast-enhanced CMR-angiography. J Cardiovasc Magn Reson. 2008;10:56.

92. Voges I, Jerosch-Herold M, Hedderich J, Pardun E, Hart C, Gabbert DD, Hansen JH, Petko C, Kramer HH, Rickers C. Normal values of aortic dimensions, distensibility, and pulse wave velocity in children and young adults: a cross-sectional study. J Cardiovasc Magn Reson. 2012;14:77.

93. Kutty S, Kuehne T, Gribben P, Reed E, Li L, Danford DA, Beerbaum PB, Sarikouch $\mathrm{S}$. Ascending aortic and main pulmonary artery areas derived from cardiovascular magnetic resonance as reference values for normal subjects and repaired tetralogy of Fallot. Circ Cardiovasc Imaging. 2012;5:644-51.

94. Dogui A, Redheuil A, Lefort M, DeCesare A, Kachenoura N, Herment A, Mousseaux E. Measurement of aortic arch pulse wave velocity in cardiovascular MR: comparison of transit time estimators and description of a new approach. J Magn Reson Imaging. 2011;33:1321-9.

95. Turkbey EB, Redheuil A, Backlund JY, Small AC, Cleary PA, Lachin JM, Lima JA, Bluemke DA, Diabetes C. Complications Trial/Epidemiology of Diabetes I, Complications Research G: Aortic distensibility in type 1 diabetes. Diabetes Care. 2013;36:2380-7.

96. Cavalcante JL, Lima JA, Redheuil A, Al-Mallah MH. Aortic stiffness: current understanding and future directions. J Am Coll Cardiol. 2011;57:1511-22.

97. Rose JL, Lalande A, Bouchot O, el Bourennane B, Walker PM, Ugolini P, Revol-Muller C, Cartier R, Brunotte F. Influence of age and sex on aortic distensibility assessed by MRI in healthy subjects. Magn Reson Imaging. 2010:28:255-63.

98. Kim EK, Chang SA, Jang SY, Kim Y, Kim SM, Oh JK, Choe YH, Kim DK. Assessment of regional aortic stiffness with cardiac magnetic resonance imaging in a healthy Asian population. Int J Cardiovasc Imaging. 2013:29(Suppl 1):57-64

99. Burman ED, Keegan J, Kilner PJ. Pulmonary artery diameters, cross sectional areas and area changes measured by cine cardiovascular magnetic resonance in healthy volunteers. J Cardiovasc Magn Reson. 2016;18:12.

100. Knobel Z, Kellenberger CJ, Kaiser T, Albisetti M, Bergstrasser E, Bueche ER. Geometry and dimensions of the pulmonary artery bifurcation in children and adolescents: assessment in vivo by contrast-enhanced MR-angiography. Int J Cardiovasc Imaging. 2011;27:385-96.

101. Gottbrecht M, Kramer CM, Salerno M. Native T1 and extracellular volume measurements by cardiac MRI in healthy adults: a meta-analysis. Radiology. 2019;290:317-26.

102. Messroghli DR, Moon JC, Ferreira VM, Grosse-Wortmann L, He T, Kellman P, Mascherbauer J, Nezafat R, Salerno M, Schelbert EB, et al. Clinical recommendations for cardiovascular magnetic resonance mapping of T1, T2, T2* and extracellular volume: A consensus statement by the Society for Cardiovascular Magnetic Resonance (SCMR) endorsed by the European Association for Cardiovascular Imaging (EACVI). J Cardiovasc Magn Reson. 2017;19:75.

103. Messroghli DR, Radjenovic A, Kozerke S, Higgins DM, Sivananthan MU, Ridgway JP. Modified Look-Locker inversion recovery (MOLLI) 
for high-resolution T1 mapping of the heart. Magn Reson Med. 2004:52:141-6.

104. Piechnik SK, Ferreira VM, Dall'Armellina E, Cochlin LE, Greiser A, Neubauer S, Robson MD. Shortened Modified Look-Locker Inversion recovery (ShMOLLI) for clinical myocardial T1-mapping at 1.5 and $3 \mathrm{~T}$ within a 9 heartbeat breathhold. J Cardiovasc Magn Reson. 2010;12:69.

105. Chow K, Flewitt JA, Green JD, Pagano JJ, Friedrich MG, Thompson RB. Saturation recovery single-shot acquisition (SASHA) for myocardial T(1) mapping. Magn Reson Med. 2014:71:2082-95.

106. Schelbert EB, Testa SM, Meier CG, Ceyrolles WJ, Levenson JE, Blair AJ, Kellman P, Jones BL, Ludwig DR, Schwartzman D, et al. Myocardial extravascular extracellular volume fraction measurement by gadolinium cardiovascular magnetic resonance in humans: slow infusion versus bolus. J Cardiovasc Magn Reson. 2011;13:16.

107. Kawel N, Nacif M, Zavodni A, Jones J, Liu S, Sibley CT, Bluemke DA. T1 mapping of the myocardium: intra-individual assessment of the effect of field strength, cardiac cycle and variation by myocardial region. J Cardiovasc Magn Reson. 2012a; 14:27.

108. Gai N, Turkbey EB, Nazarian S, van der Geest RJ, Liu CY, Lima JA, Bluemke DA. T1 mapping of the gadolinium-enhanced myocardium: adjustment for factors affecting interpatient comparison. Magn Reson Med. 2011;65:1407-15.

109. Kawel N, Nacif M, Zavodni A, Jones J, Liu S, Sibley CT, Bluemke DA T1 mapping of the myocardium: intra-individual assessment of postcontrast $\mathrm{T} 1$ time evolution and extracellular volume fraction at $3 \mathrm{~T}$ for Gd-DTPA and Gd-BOPTA. J Cardiovasc Magn Reson. 2012b;14:26.

110. Lee J, Liu S, Nacif MS, Ugander M, Han J, Kawel N, Sibley CT, Kellman P, Arai AE, Bluemke DA. Myocardial T1 and extracellular volume fraction mapping at 3 tesla. J Cardiovasc Magn Reson. 2011;13:75.

111. Puntmann VO, D'Cruz D, Smith Z, Pastor A, Choong P, Voigt T, Carr-White G, Sangle S, Schaeffter T, Nagel E. Native myocardial T1 mapping by cardiovascular magnetic resonance imaging in subclinical cardiomyopathy in patients with systemic lupus erythematosus. Circ Cardiovasc Imaging. 2013;6:295-301.

112. Ugander M, Oki AJ, Hsu LY, Kellman P, Greiser A, Aletras AH, Sibley CT, Chen MY, Bandettini WP, Arai AE. Extracellular volume imaging by magnetic resonance imaging provides insights into overt and sub-clinical myocardial pathology. Eur Heart J. 2012;33:1268-78.

113. White SK, Sado DM, Flett AS, Moon JC. Characterising the myocardial interstitial space: the clinical relevance of non-invasive imaging. Heart. 2012;98:773-9.

114. Arheden H, Saeed M, Higgins CB, Gao DW, Ursell PC, Bremerich J, Wyttenbach R, Dae MW, Wendland MF. Reperfused rat myocardium subjected to various durations of ischemia: estimation of the distribution volume of contrast material with echo-planar MR imaging. Radiology. 2000:215:520-8.

115. Kellman P, Wilson JR, Xue H, Ugander M, Arai AE. Extracellular volume fraction mapping in the myocardium, part 1: evaluation of an automated method. J Cardiovasc Magn Reson. 2012;14:63.

116. Piechnik SK, Ferreira VM, Lewandowski AJ, Ntusi NA, Banerjee R, Holloway C, Hofman MB, Sado DM, Maestrini V, White SK, et al. Normal variation of magnetic resonance T1 relaxation times in the human population at 1.5 T using ShMOLLI. J Cardiovasc Magn Reson. 2013;15:13.

117. Dabir D, Child N, Kalra A, Rogers T, Gebker R, Jabbour A, Plein S, Yu CY, Otton J, Kidambi A, et al. Reference values for healthy human myocardium using a T1 mapping methodology: results from the Internationa T1 Multicenter cardiovascular magnetic resonance study. J Cardiovasc Magn Reson. 2014;16:69.

118. Reiter U, Reiter G, Dorr K, Greiser A, Maderthaner R, Fuchsjager M. Normal diastolic and systolic myocardial T1 values at 1.5-T MR imaging: correlations and blood normalization. Radiology. 2014;271:365-72.

119. Kawel N, Nacif M, Santini F, Liu S, Bremerich J, Arai AE, Bluemke DA. Partition coefficients for gadolinium chelates in the normal myocardium: comparison of gadopentetate dimeglumine and gadobenate dimeglumine. J Magn Reson Imaging. 2012;36:733-7.

120. Fontana M, White SK, Banypersad SM, Sado DM, Maestrini V, Flett AS, Piechnik SK, Neubauer S, Roberts N, Moon JC. Comparison of T1 mapping techniques for ECV quantification. Histological validation and reproducibility of ShMOLLI versus multibreath-hold T1 quantification equilibrium contrast CMR. J Cardiovasc Magn Reson. 2012;14:88.
121. Kellman P, Wilson JR, Xue H, Bandettini WP, Shanbhag SM, Druey KM, Ugander M, Arai AE. Extracellular volume fraction mapping in the myocardium, part 2: initial clinical experience. J Cardiovasc Magn Reson. 2012;14:64

122. Sado DM, White SK, Piechnik SK, Banypersad SM, Treibel T, Captur G, Fontana M, Maestrini V, Flett AS, Robson MD, et al. Identification and assessment of Anderson-Fabry disease by cardiovascular magnetic resonance noncontrast myocardial T1 mapping. Circ Cardiovasc Imaging. 2013;6:392-8.

123. Ferreira VM, Piechnik SK, Dall'Armellina E, Karamitsos TD, Francis JM, Ntusi N, Holloway C, Choudhury RP, Kardos A, Robson MD, et al. Native T1-mapping detects the location, extent and patterns of acute myocarditis without the need for gadolinium contrast agents. J Cardiovasc Magn Reson. 2014;16:36.

124. Fontana M, Banypersad SM, Treibel TA, Maestrini V, Sado DM, White SK, Pica S, Castelletti S, Piechnik SK, Robson MD, et al. Native T1 mapping in transthyretin amyloidosis. JACC Cardiovasc Imaging. 2014;7:157-65.

125. Liu CY, Bluemke DA, Gerstenblith G, Zimmerman SL, Li J, Zhu H, Lai S, Lai H. Reference values of myocardial structure, function, and tissue composition by cardiac magnetic resonance in healthy AfricanAmericans at $3 \mathrm{~T}$ and their relations to serologic and cardiovascular risk factors. Am J Cardiol. 2014;114:789-95.

126. Puntmann VO, Arroyo Ucar E, Hinojar Baydes R, Ngah NB, Kuo YS, Dabir D, Macmillan A, Cummins C, Higgins DM, Gaddum N, et al. Aortic stiffness and interstitial myocardial fibrosis by native $\mathrm{T1}$ are independently associated with left ventricular remodeling in patients with dilated cardiomyopathy. Hypertension. 2014;64:762-8.

127. Siepen F, Buss SJ, Messroghli D, Andre F, Lossnitzer D, Seitz S, Keller M, Schnabel PA, Giannitsis E, Korosoglou G, et al. T1 mapping in dilated cardiomyopathy with cardiac magnetic resonance: quantification of diffuse myocardial fibrosis and comparison with endomyocardial biopsy. Eur Heart J Cardiovasc Imaging. 2015;16:210-6.

128. Banypersad SM, Fontana M, Maestrini V, Sado DM, Captur G, Petrie A, Piechnik SK, Whelan CJ, Herrey AS, Gillmore JD, et al. T1 mapping and survival in systemic light-chain amloidosis. Eur Heart J. 2015;36:244-51.

129. Edwards NC, Moody WE, Yuan M, Hayer MK, Ferro CJ, Townend JN, Steeds RP. Diffuse interstitial fibrosis and myocardial dysfunction in early chronic kidney disease. Am J Cardiol. 2015;115:1311-7.

130. Fontana M, Banypersad SM, Treibel TA, Abdel-Gadir A, Maestrini V, Lane T, Gilbertson JA, Hutt DF, Lachmann HJ, Whelan CJ, et al. Differential myocyte responses in patients with cardiac transthyretin amyloidosis and light-chain amyloidosis: a cardiac MR Imaging Study. Radiology. 2015;277:388-97.

131. Treibel TA, Zemrak F, Sado DM, Banypersad SM, White SK, Maestrini V, Barison A, Patel V, Herrey AS, Davies C, et al. Extracellular volume quantification in isolated hypertension-changes at the detectable limits? J Cardiovasc Magn Reson. 2015;17:74.

132. Goebel J, Seifert I, Nensa F, Schemuth HP, Maderwald S, Quick HH, Schlosser T, Jensen C, Bruder O, Nassenstein K. Can native t1 mapping differentiate between healthy and diffuse diseased myocardium in clinical routine cardiac MR imaging? PLOS ONE. 2016;11:e0155591.

133. Gormeli CA, Gormeli G, Yagmur J, Ozdemir ZM, Kahraman AS, Colak C, Ozdemir R. Assessment of myocardial changes in athletes with native T1 mapping and cardiac functional evaluation using 3 T MRI. Int J Cardiovasc Imaging. 2016;32:975-81.

134. Hinojar R, Foote L, Sangle S, Marber M, Mayr M, Carr-White G, D'Cruz $D$, Nagel E, Puntmann VO. Native T1 and T2 mapping by CMR in lupus myocarditis: Disease recognition and response to treatment. Int J Cardiol. 2016;222:717-26.

135. Ntusi N, O'Dwyer E, Dorrell L, Wainwright E, Piechnik S, Clutton G, Hancock G, Ferreira V, Cox P, Badri M, et al. HIV-1-related cardiovascular disease is associated with chronic inflammation, frequent pericardial effusions, and probable myocardial edema. Circ Cardiovasc Imaging. 2016:9:e004430

136. Rauhalammi SM, Mangion K, Barrientos PH, Carrick DJ, Clerfond G, McClure J, McComb C, Radjenovic A, Berry C. Native myocardial longitudinal ( $\mathrm{T} 1$ ) relaxation time: Regional, age, and sex associations in the healthy adult heart. J Magn Reson Imaging. 2016;44:541-8.

137. Costello BT, Springer F, Hare JL, Gerche A, lles L, Ellims AH, Schmitt B, Taylor AJ. SASHA versus ShMOLLI: a comparison of T1 mapping 
methods in health and dilated cardiomyopathy at 3 T. Int J Cardiovasc Imaging. 2017;33:1551-60.

138. Avitzur N, Satriano A, Afzal M, Narous M, Mikami Y, Hansen R, Dobko G, Flewitt J, Lydell CP, Howarth AG, et al. 3D myocardial deformation analysis from cine MRI as a marker of amyloid protein burden in cardiac amyloidosis: validation versus T1 mapping. Int I Cardiovasc Imaging. 2018;34:1937-46

139. Doerner J, Eichhorn L, Luetkens JA, Lunkenheimer JN, Albers J, Nadal J, Schild HH, Naehle CP. Effects of repetitive prolonged breath-hold in elite divers on myocardial fibrosis and cerebral morphology. Eur J Radiol. 2018;103:13-8.

140. Guo Q, Wu LM, Wang Z, Shen JY, Su X, Wang CQ, Gong XR, Yan QR, He $\mathrm{Q}$, Zhang W, et al. Early detection of silent myocardial impairment in drug-naive patients with new-onset systemic lupus erythematosus: a three-center prospective study. Arthritis Rheumatol. 2018;70:2014-24.

141. Ridouani F, Damy T, Tacher V, Derbel H, Legou F, Sifaoui I, Audureau E, Bodez D, Rahmouni A, Deux JF. Myocardial native T2 measurement to differentiate light-chain and transthyretin cardiac amyloidosis and assess prognosis. J Cardiovasc Magn Reson. 2018;20:58.

142. Rosmini S, Bulluck H, Captur G, Treibel TA, Abdel-Gadir A, Bhuva AN, Culotta V, Merghani A, Fontana M, Maestrini V, et al. Myocardial native $\mathrm{T} 1$ and extracellular volume with healthy ageing and gender. Eur Heart J Cardiovasc Imaging. 2018;19:615-21.

143. Shang $Y$, Zhang $X$, Zhou $X$, Wang J. Extracellular volume fraction measurements derived from the longitudinal relaxation of blood-based synthetic hematocrit may lead to clinical errors in $3 \mathrm{~T}$ cardiovascular magnetic resonance. J Cardiovasc Magn Reson. 2018;20:56.

144. Yang D, Li X, Sun JY, Cheng W, Greiser A, Zhang TJ, Liu H, Wan K, Luo Y, An $Q$, et al. Cardiovascular magnetic resonance evidence of myocardial fibrosis and its clinical significance in adolescent and adult patients with Ebstein's anomaly. J Cardiovasc Magn Reson. 2018:20:69.

145. Granitz M, Motloch LJ, Granitz C, Meissnitzer M, Hitzl W, Hergan K, Schlattau A. Comparison of native myocardial T1 and T2 mapping at 1.5T and $3 \mathrm{~T}$ in healthy volunteers: Reference values and clinical implications. Wien Klin Wochenschr. 2019;131:143-155.

146. Imran M, Wang L, McCrohon J, Yu C, Holloway C, Otton J, Huang J, Stehning C, Moffat KJ, Ross J, et al. Native T1 mapping in the diagnosis of cardiac allograft rejection: a prospective histologically validated study. JACC Cardiovasc Imaging. 2019;12:1618-28.

147. Lehmonen L, Kaasalainen T, Atula S, Mustonen T, Holmstrom M. Myocardial tissue characterization in patients with hereditary gelsolin (AGel) amyloidosis using novel cardiovascular magnetic resonance techniques. Int J Cardiovasc Imaging. 2019;35:351-8.

148. Vijapurapu R, Nordin S, Baig S, Liu B, Rosmini S, Augusto J, Tchan M, Hughes DA, Geberhiwot T, Moon JC, et al. Global longitudina strain, myocardial storage and hypertrophy in Fabry disease. Heart. 2019;105:470-6.

149. Wan K, Li W, Sun J, Xu Y, Wang J, Liu H, Dong Y, Cheng W, Zhang Q, Zeng $Z$, et al. Regional amyloid distribution and impact on mortality in lightchain amyloidosis: a T1 mapping cardiac magnetic resonance study. Amyloid. 2019;26:45-51.

150. Brittain JH, Hu BS, Wright GA, Meyer CH, Macovski A, Nishimura DG. Coronary angiography with magnetization-prepared T2 contrast. Magn Reson Med. 1995;33:689-96.

151. Giri S, Chung YC, Merchant A, Mihai G, Rajagopalan S, Raman SV, Simonetti OP. T2 quantification for improved detection of myocardial edema. $J$ Cardiovasc Magn Reson. 2009;11:56.

152. Kamath R, Gottbrecht M, Salerno M. T2 relatxation times in healthy adults: a meta-analysis. Abstract submitted to SCMR 23rd Annual Scientific Session; 2019

153. Roy C, Slimani A, de Meester C, Amzulescu M, Pasquet A, Vancraeynest D, Vanoverschelde JL, Pouleur AC, Gerber BL. Age and sex corrected normal reference values of $\mathrm{T} 1, \mathrm{~T} 2 \mathrm{~T} 2^{*}$ and $\mathrm{ECV}$ in healthy subjects at $3 \mathrm{~T}$ CMR. J Cardiovasc Magn Reson. 2017:19:72.

154. von Knobelsdorff-Brenkenhoff F, Prothmann M, Dieringer MA, Wassmuth R, Greiser A, Schwenke C, Niendorf T, Schulz-Menger J. Myocardial $\mathrm{T} 1$ and $\mathrm{T} 2$ mapping at $3 \mathrm{~T}$ : reference values, influencing factors and implications. J Cardiovasc Magn Reson. 2013;15:53.

155. Wassmuth R, Prothmann M, Utz W, Dieringer M, von KnobelsdorffBrenkenhoff F, Greiser A, Schulz-Menger J. Variability and homogeneity of cardiovascular magnetic resonance myocardial T2-mapping in volunteers compared to patients with edema. J Cardiovasc Magn Reson. 2013;15:27.

156. Pennell DJ. T2* magnetic resonance: iron and gold. JACC Cardiovasc Imaging. 2008;1:579-81.

157. Anderson LJ, Holden S, Davis B, Prescott E, Charrier CC, Bunce NH, Firmin DN, Wonke B, Porter J, Walker JM, Pennell DJ. Cardiovascular T2-star (T2*) magnetic resonance for the early diagnosis of myocardial iron overload. Eur Heart J. 2001;22:2171-9.

158. Pennell DJ, Udelson JE, Arai AE, Bozkurt B, Cohen AR, Galanello R, Hoffman TM, Kiernan MS, Lerakis S, Piga A, et al. Cardiovascular function and treatment in beta-thalassemia major: a consensus statement from the American Heart Association. Circulation. 2013;128:281-308.

159. Wood JC, Ghugre N. Magnetic resonance imaging assessment of excess iron in thalassemia, sickle cell disease and other iron overload diseases. Hemoglobin. 2008;32:85-96.

160. Carpenter JP, He T, Kirk P, Roughton M, Anderson LJ, de Noronha SV, Sheppard MN, Porter JB, Walker JM, Wood JC, et al. On T2* magnetic resonance and cardiac iron. Circulation. 2011:123:1519-28.

161. Kirk P, Smith GC, Roughton M, He T, Pennell DJ. Myocardial T2* is not affected by ageing, myocardial fibrosis, or impaired left ventricular function. J Magn Reson Imaging. 2010;32:1095-8.

162. Kirk P, Roughton M, Porter JB, Walker JM, Tanner MA, Patel J, Wu D, Taylor J, Westwood MA, Anderson LJ, Pennell DJ. Cardiac T2* magnetic resonance for prediction of cardiac complications in thalassemia major. Circulation. 2009;120:1961-8.

163. Arts T, Prinzen FW, Delhaas T, Milles JR, Rossi AC, Clarysse P. Mapping displacement and deformation of the heart with local sine-wave modeling. IEEE Trans Med Imaging. 2010;29:1114-23.

164. Cupps BP, Taggar AK, Reynolds LM, Lawton JS, Pasque MK. Regional myocardial contractile function: multiparametric strain mapping. Interact Cardiovasc Thorac Surg. 2010:10:953-7.

165. Del-Canto I, Lopez-Lereu MP, Monmeneu JV, Croisille P, Clarysse P, Chorro FJ, Bodi V, Moratal D. Characterization of normal regional myocardial function by MRI cardiac tagging. J Magn Reson Imaging. 2015:41:83-92.

166. el Ibrahim SH. Myocardial tagging by cardiovascular magnetic resonance: evolution of techniques-pulse sequences, analysis algorithms, and applications. J Cardiovasc Magn Reson. 2011;13:36.

167. Schuster A, Hor KN, Kowallick JT, Beerbaum P, Kutty S. Cardiovascular magnetic resonance myocardial feature tracking: concepts and clinical applications. Circ Cardiovasc Imaging. 2016;9:e004077.

168. Petitjean C, Rougon N, Cluzel P. Assessment of myocardial function: a review of quantification methods and results using tagged MRI. J Cardiovasc Magn Reson. 2005;7:501-16.

169. Miller CA, Borg A, Clark D, Steadman CD, McCann GP, Clarysse P, Croisille $P$, Schmitt M. Comparison of local sine wave modeling with harmonic phase analysis for the assessment of myocardial strain. J Magn Reson Imaging. 2013;38:320-8.

170. Bogaert J, Rademakers FE. Regional nonuniformity of normal adult human left ventricle. Am J Physiol Heart Circ Physiol. 2001;280:H610-620

171. Jeung MY, Germain P, Croisille P. Myocardial tagging with MR imaging: overview of normal and pathologic findings. Radiographics. 2012:32:1381-98

172. Piella G, De Craene M, Bijnens BH, Tobon-Gomez C, Huguet M, Avegliano G, Frangi AF. Characterizing myocardial deformation in patients with left ventricular hypertrophy of different etiologies using the strain distribution obtained by magnetic resonance imaging. Rev Esp Cardiol. 2010;63:1281-91.

173. Castillo E, Osman NF, Rosen BD, El-Shehaby I, Pan L, Jerosch-Herold M, Lai S, Bluemke DA, Lima JA. Quantitative assessment of regional myocardial function with MR-tagging in a multi-center study: interobserver and intraobserver agreement of fast strain analysis with Harmonic Phase (HARP) MRI. J Cardiovasc Magn Reson. 2005;7:783-91.

174. Moore CC, Lugo-Olivieri CH, McVeigh ER, Zerhouni EA. Three-dimensional systolic strain patterns in the normal human left ventricle: characterization with tagged MR imaging. Radiology. 2000;214:453-66.

175. Claus P, Omar AMS, Pedrizzetti G, Sengupta PP, Nagel E. Tissue tracking technology for assessing cardiac mechanics: principles, normal values, and clinical applications. JACC Cardiovasc Imaging. 2015;8:1444-60. 
176. Peng J, Zhao X, Zhao L, Fan Z, Wang Z, Chen H, Leng S, Allen J, Tan RS, Koh AS, et al. Normal values of myocardial deformation assessed by cardiovascular magnetic resonance feature tracking in a healthy Chinese population: a multicenter study. Front Physiol. 2018;9:1181.

177. Augustine D, Lewandowski AJ, Lazdam M, Rai A, Francis J, Myerson S, Noble A, Becher H, Neubauer S, Petersen SE, Leeson P. Global and regional left ventricular myocardial deformation measures by magnetic resonance feature tracking in healthy volunteers: comparison with tagging and relevance of gender. J Cardiovasc Magn Reson. 2013;15:8.

178. Oxenham HC, Young AA, Cowan BR, Gentles TL, Occleshaw CJ, Fonseca CG, Doughty RN, Sharpe N. Age-related changes in myocardial relaxation using three-dimensional tagged magnetic resonance imaging. J Cardiovasc Magn Reson. 2003;5:421-30.

179. Andre F, Steen H, Matheis P, Westkott M, Breuninger K, Sander $Y$, Kammerer R, Galuschky C, Giannitsis E, Korosoglou G, et al. Age- and gender-related normal left ventricular deformation assessed by cardiovascular magnetic resonance feature tracking. J Cardiovasc Magn Reson. 2015;17:25

180. Lawton JS, Cupps BP, Knutsen AK, Ma N, Brady BD, Reynolds LM, Pasque MK. Magnetic resonance imaging detects significant sex differences in human myocardial strain. Biomed Eng Online. 2011;10:76.

181. Shehata ML, Cheng S, Osman NF, Bluemke DA, Lima JA. Myocardial tissue tagging with cardiovascular magnetic resonance. J Cardiovasc Magn Reson. 2009;11:55.

182. Neizel M, Lossnitzer D, Korosoglou G, Schaufele T, Lewien A, Steen $H$, Katus HA, Osman NF, Giannitsis E. Strain-encoded (SENC) magnetic resonance imaging to evaluate regional heterogeneity of myocardial strain in healthy volunteers: Comparison with conventional tagging. J Magn Reson Imaging. 2009;29:99-105.

183. Venkatesh BA, Donekal S, Yoneyama K, Wu C, Fernandes VR, Rosen BD, Shehata ML, McClelland R, Bluemke DA, Lima JA. Regional myocardial functional patterns: quantitative tagged magnetic resonance imaging in an adult population free of cardiovascular risk factors: the multi-ethnic study of atherosclerosis (MESA). J Magn Reson Imaging. 2015:42:153-9.

184. Cernicanu A, Axel L. Theory-based signal calibration with single-point T1 measurements for first-pass quantitative perfusion MRI studies. Acad Radiol. 2006;13:686-93.

185. Gatehouse PD, Elkington AG, Ablitt NA, Yang GZ, Pennell DJ, Firmin DN. Accurate assessment of the arterial input function during high-dose myocardial perfusion cardiovascular magnetic resonance. J Magn Reson Imaging. 2004:20:39-45.

186. Christian TF, Rettmann DW, Aletras AH, Liao SL, Taylor JL, Balaban RS, Arai AE. Absolute myocardial perfusion in canines measured by using dual-bolus first-pass MR imaging. Radiology. 2004;232:677-84.

187. Ishida M, Schuster A, Morton G, Chiribiri A, Hussain S, Paul M, Merkle N, Steen H, Lossnitzer D, Schnackenburg B, et al. Development of a universal dual-bolus injection scheme for the quantitative assessment of myocardial perfusion cardiovascular magnetic resonance. J Cardiovasc Magn Reson. 2011;13:28.

188. Vasu S, Bandettini WP, Hsu LY, Kellman P, Leung S, Mancini C, Shanbhag SM, Wilson J, Booker OJ, Arai AE. Regadenoson and adenosine are equivalent vasodilators and are superior than dipyridamole-a study of first pass quantitative perfusion cardiovascular magnetic resonance. J Cardiovasc Magn Reson. 2013;15:85.

189. Fairbairn TA, Motwani M, Mather AN, Biglands JD, Larghat AM, Radjenovic A, Greenwood JP, Plein S. Cardiac MR imaging to measure myocardial blood flow response to the cold pressor test in healthy smokers and nonsmokers. Radiology. 2014;270:82-90.

190. Weng AM, Wilimsky S, Bender G, Hahner S, Kostler H, Ritter CO. Magnetic resonance cold pressor test to investigate potential endothelial dysfunction in patients suffering from type 1 diabetes. J Magn Reson Imaging. 2018:48:1595-601.

191. Jerosch-Herold M, Seethamraju RT, Swingen CM, Wilke NM, Stillman AE. Analysis of myocardial perfusion MRI. J Magn Reson Imaging. 2004;19:758-70

192. Wang L, Jerosch-Herold M, Jacobs DR Jr, Shahar E, Folsom AR. Coronary risk factors and myocardial perfusion in asymptomatic adults: the Multi-Ethnic Study of Atherosclerosis (MESA). J Am Coll Cardiol. 2006;47:565-72.
193. Chareonthaitawee P, Kaufmann PA, Rimoldi O, Camici PG. Heterogeneity of resting and hyperemic myocardial blood flow in healthy humans. Cardiovasc Res. 2001;50:151-61.

194. Moro PJ, Flavian A, Jacquier A, Kober F, Quilici J, Gaborit B, Bonnet $J \mathrm{~L}$, Moulin G, Cozzone PJ, Bernard M. Gender differences in response to cold pressor test assessed with velocity-encoded cardiovascular magnetic resonance of the coronary sinus. J Cardiovasc Magn Reson. 2011;13:54.

195. Brown LAE, Onciul SC, Broadbent DA, Johnson K, Fent GJ, Foley JRJ, Garg P, Chew PG, Knott K, Dall'Armellina E, et al. Fully automated, inline quantification of myocardial blood flow with cardiovascular magnetic resonance: repeatability of measurements in healthy subjects. J Cardiovasc Magn Reson. 2018;20:48.

196. Madriago E, Wells R, Sahn DJ, Diggs BS, Langley SM, Woodward DJ, Jerosch-Herold M, Silberbach M. Abnormal myocardial blood flow in children with mild/moderate aortic stenosis. Cardiol Young. 2015;25:1358-66.

197. Bhuva AN, Bai W, Lau C, Ye Y, Bulluck H, McAlindon E, Culotta V, Swoboda PP, Captur G, et al. A multicenter, scan-rescan, human and machine learning CMR study to test generalizability and precision in imaging biomarker analysis. Circ Cardiovasc Imaging. 2019;12:e009214.

198. Bernard O, Lalande A, Zotti C, Cervenansky F, Yang X, Heng PA, Cetin I, Lekadir K, Camara O, Gonzalez Ballester MA, et al. Deep learning techniques for automatic MRI cardiac multi-structures segmentation and diagnosis: is the problem solved? IEEE Trans Med Imaging. 2018;37:2514-25.

199. Curiale AH, Colavecchia FD, Mato G. Automatic quantification of the LV function and mass: a deep learning approach for cardiovascular MRI. Comput Methods Programs Biomed. 2019;169:37-50.

200. Khened M, Kollerathu VA, Krishnamurthi G. Fully convolutional multiscale residual DenseNets for cardiac segmentation and automated cardiac diagnosis using ensemble of classifiers. Med Image Anal. 2019;51:21-45

201. Tan LK, McLaughlin RA, Lim E, Abdul Aziz YF, Liew YM. Fully automated segmentation of the left ventricle in cine cardiac MRI using neural network regression. J Magn Reson Imaging. 2018;48:140-52.

202. Tong Q, Li C, Si W, Liao X, Tong Y, Yuan Z, Heng PA. RIANet: Recurrent interleaved attention network for cardiac MRI segmentation. Comput Biol Med. 2019;109:290-302.

203. Tran PV. A fully convolutional neural network for cardiac segmentation in short-axis MRI. arXiv preprint. 2016.

204. Vigneault DM, Xie W, Ho CY, Bluemke DA, Noble JA. Omega-Net (Omega-Net): fully automatic, multi-view cardiac MR detection, orientation, and segmentation with deep neural networks. Med Image Anal. 2018;48:95-106.

205. Campello VM, Martín-Isla C, Izquierdo C, Petersen SE, Ballester MAG, Lekadir K. Combining multi-sequence and synthetic images for improved segmentation of late gadolinium enhancement cardiac MRI. arXiv preprint. 2019.

206. Moccia S, Banali R, Martini C, Muscogiuri G, Pontone G, Pepi M, Caiani EG. Development and testing of a deep learning-based strategy for scar segmentation on CMR-LGE images. MAGMA. 2019;32:187-95.

207. Fahmy AS, El-Rewaidy H, Nezafat M, Nakamori S, Nezafat R. Automated analysis of cardiovascular magnetic resonance myocardial native T1 mapping images using fully convolutional neural networks. J Cardiovasc Magn Reson. 2019;21:7.

208. Bratt A, Kim J, Pollie M, Beecy AN, Tehrani NH, Codella N, Perez-Johnston R, Palumbo MC, Alakbarli J, Colizza W, et al. Machine learning derived segmentation of phase velocity encoded cardiovascular magnetic resonance for fully automated aortic flow quantification. J Cardiovasc Magn Reson. 2019;21:1.

209. Zheng Q, Delingette H, Ayache N. Explainable cardiac pathology classification on cine MRI with motion characterization by semi-supervised learning of apparent flow. Med Image Anal. 2019;56:80-95.

210. Ronneberger O, Fischer P, Brox T. U-net: Convolutional networks for biomedical image segmentation. In: International Conference on Medical image computing and computer-assisted intervention. Springer; 2015. p. 234-241.

211. Grand challenges-All Challenges. https://grand-challenge.org/chall enges/. Accessed 17 Oct 2019. 
212. Backhaus SJ, Staab W, Steinmetz M, Ritter CO, Lotz J, Hasenfuss G, Schuster A, Kowallick JT. Fully automated quantification of biventricular volumes and function in cardiovascular magnetic resonance: applicability to clinical routine settings. J Cardiovasc Magn Reson. 2019;21:24.

213. Radau P, Lu Y, Connelly K, Paul G, Dick A, Wright G. Evaluation framework for algorithms segmenting short axis cardiac MRI. In: The MIDAS Journal-Cardiac MR Left Ventricle Segmentation Challenge. 2009. p. 49.

214. Suinesiaputra A, Cowan BR, Finn JP, Fonseca CG, Kadish AH, Lee DC, Medrano-Gracia P, Warfield SK, Tao W, Young AA. Left ventricular segmentation challenge from cardiac MRI: a collation study. In: International Workshop on Statistical Atlases and Computational Models of the Heart. Springer; 2011. p. 88-97.

215. Petitjean C, Zuluaga MA, Bai W, Dacher J-N, Grosgeorge D, Caudron J, Ruan S, Ayed IB, Cardoso MJ, Chen H-C. Right ventricle segmentation from cardiac MRI: a collation study. Med Image Anal. 2015;19:187-202.
216. Booz Allen Hamilton Inc., Kaggle, 2015. Second annual data science bowl. https://www.kaggle.com/c/second-annual-data-science-bowl. Accessed 17 Oct 2019.

217. Fonseca CG, Backhaus M, Bluemke DA, Britten RD, Chung JD, Cowan BR, Dinov ID, Finn JP, Hunter PJ, Kadish AH. The Cardiac Atlas Project—an imaging database for computational modeling and statistical atlases of the heart. Bioinformatics. 2011;27:2288-95.

\section{Publisher's Note}

Springer Nature remains neutral with regard to jurisdictional claims in published maps and institutional affiliations.
Ready to submit your research? Choose BMC and benefit from:

- fast, convenient online submission

- thorough peer review by experienced researchers in your field

- rapid publication on acceptance

- support for research data, including large and complex data types

- gold Open Access which fosters wider collaboration and increased citations

- maximum visibility for your research: over $100 \mathrm{M}$ website views per year

At BMC, research is always in progress.

Learn more biomedcentral.com/submissions 